Supporting Information

\title{
The Synthesis and Characterization of Highly Fluorescent Polycyclic Azaborine Chromophores
}

Carl Jacky Saint-Louis, ${ }^{\dagger}$ Lacey L. Magill, ${ }^{\dagger}$ Julie A. Wilson, ${ }^{\dagger}$ Andrew R. Schroeder, ${ }^{\dagger}, \perp$ Sarah E. Harrell, ${ }^{\dagger}$ Nicolle S. Jackson, ${ }^{\dagger}$ Jamie A. Trindell, ${ }^{\dagger},{ }^{\uparrow}$ Saraphina Kim ${ }^{\dagger}$ Alexander R. Fisch, ${ }^{\dagger}, "$ Lyndsay Munro, ${ }^{\ddagger}$ Vincent J. Catalano, ${ }^{\star}$ Charles Edwin Webster, ${ }^{\S}$ Pamela P. Vaughan, ${ }^{\dagger}$ Karen S. Molek, ${ }^{\dagger}$ Alan K. Schrock, ${ }^{\dagger} * *$ and Michael T. Huggins ${ }^{\dagger, *}$

†Department of Chemistry, University of West Florida, Pensacola, Florida 32514, United States

tDepartment of Chemistry, University of Nevada, Reno, Reno, Nevada 89557, United States

${ }^{\S}$ Department of Chemistry, Mississippi State University, Mississippi State, Mississippi 39762, United States

${ }^{{ }}$School of Medicine, University of Alabama, 1825 University Boulevard, Birmingham, Alabama 35294, United States

IDepartment of Chemistry, University of Texas-Austin, 120 Inner Campus Dr., Austin, Texas 78712, United States

"Department of Chemistry, University of Tennessee, 1420 Circle Dr. Knoxville, Tennessee 37996, United States

* Michael T. Huggins. E-mail.: mhuggins@uwf.edu

* Alan K. Schrock. E-mail: aschrock@uwf.edu

1. ${ }^{1} \mathrm{H} \mathrm{NMR} /{ }^{13} \mathrm{C}$ NMR

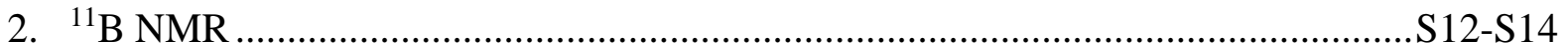

3. FT-IR S15-S19

4. Normalized UV-vis spectra of compounds 1-10 in chloroform, methanol, DMSO, acetonitrile and hexanes S20-S24

5. Normalized emission spectra of compounds 5-10 in chloroform S25-S26

6. Normalized emission spectra of compounds 1-10 in the solid state $\mathrm{S} 27$

7. Normalized UV-visible and emission spectra of compounds 5-10 in chloroform ......... S28

8. Normalized excitation spectra of compounds 3-10 in the solid state ......................... S29

9. Table S1. B3LYP/6-311 ${ }^{++} \mathrm{G}^{* *}$ molecular orbital calculations of 1-12 ....................... S30

10. Table S2. Stokes shift of compounds 3-10 in chloroform, methanol, DMSO, and

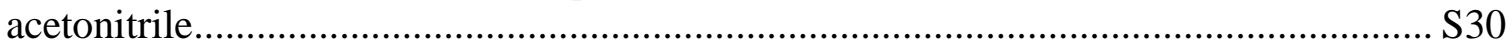

11. Table S3. Emission of 3-10 in the solid state....................................................... S30

12. B3LYP/6-311 ${ }^{++} \mathrm{G}^{* *}$ molecular orbital calculations of $\mathbf{2 0}$ and $\mathbf{2 1}$........................S31-S33

13. B3LYP/6-311 ${ }^{++} \mathrm{G}^{* *}$ molecular orbital calculations of 1-10 ..............................S34-S48

14. Simulated UV-visible spectra of compounds 1-10 and 20, 21 in the gas phase from TDB3LYP/6-31G(d').

S49-S50

15. Simulated UV-visible (absorption) and emission spectra of compounds $\mathbf{1 , 3 - 1 0}$ in the gas phase from TD-B3LYP/6-31G(d')

16. B3LYP/6-31G(d') coordinates for compounds 1-10 . S54-S56

17. TD-B3LYP/6-31G(d') coordinates for compounds 1,3-10 S57-S59

18. Thermal ellipsoid plot for compound $\mathbf{4}$ and compound $\mathbf{6}$. S60-S61

19. X-Ray Crystal data for compounds 4 and $\mathbf{6}$. S62-S69 


\section{${ }^{1} \mathrm{H}$ NMR $/{ }^{13} \mathrm{C}$ NMR}

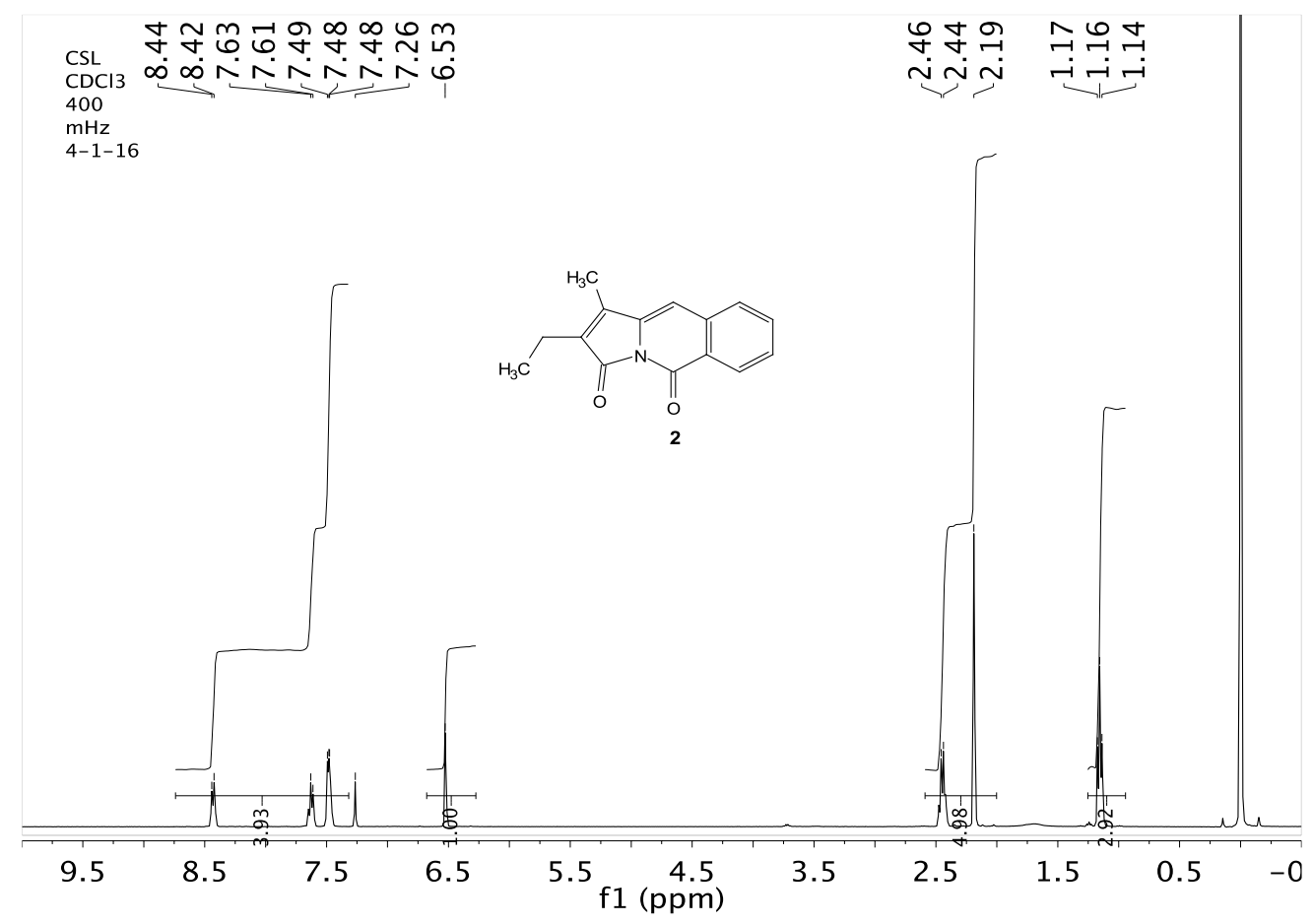

${ }^{1} \mathrm{H}$ NMR spectrum (400 MHz) of 2 in $\mathrm{CDCl}_{3}$

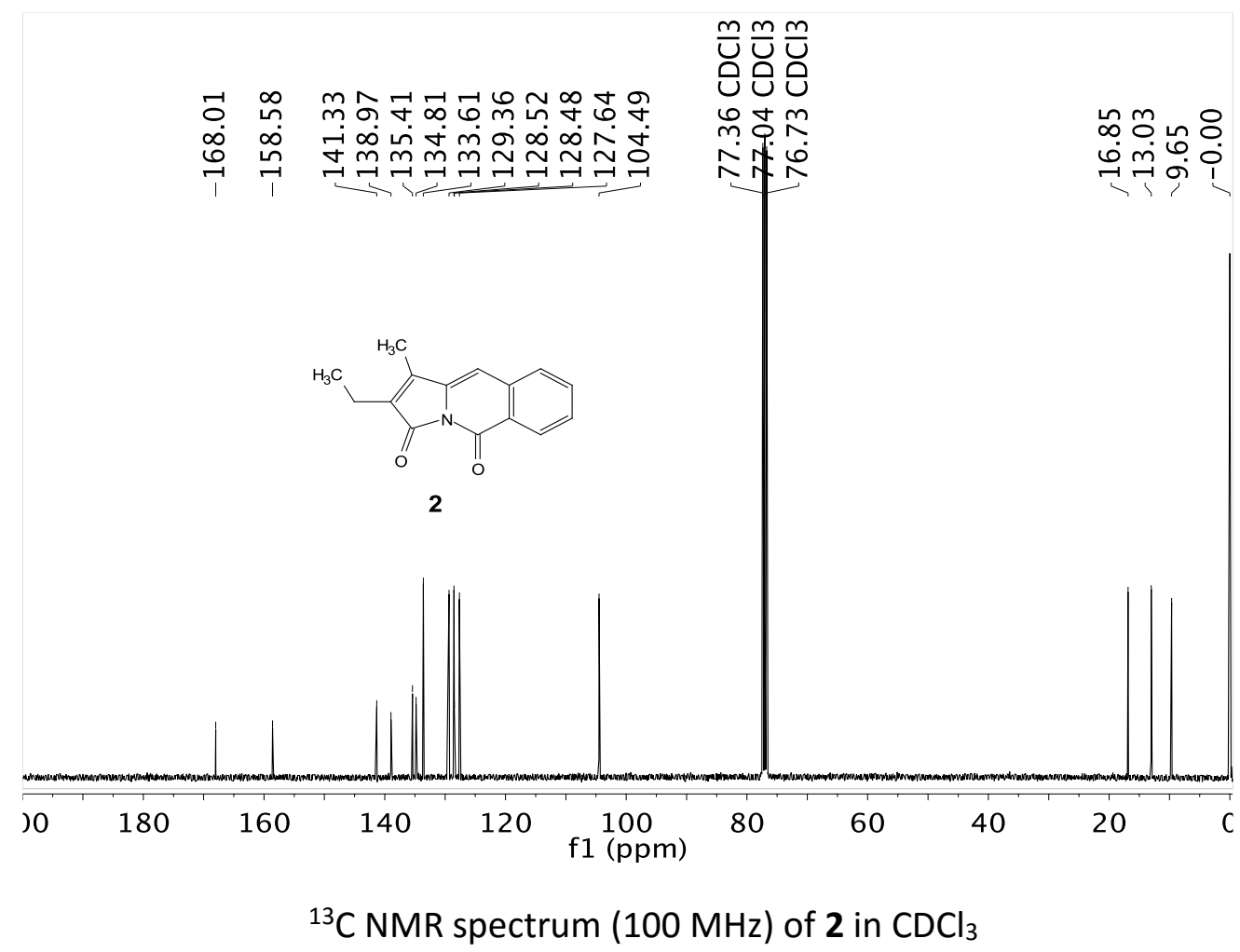




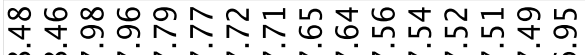

$\infty \infty N \wedge N \wedge N \wedge N \wedge N \wedge N \wedge N 6$

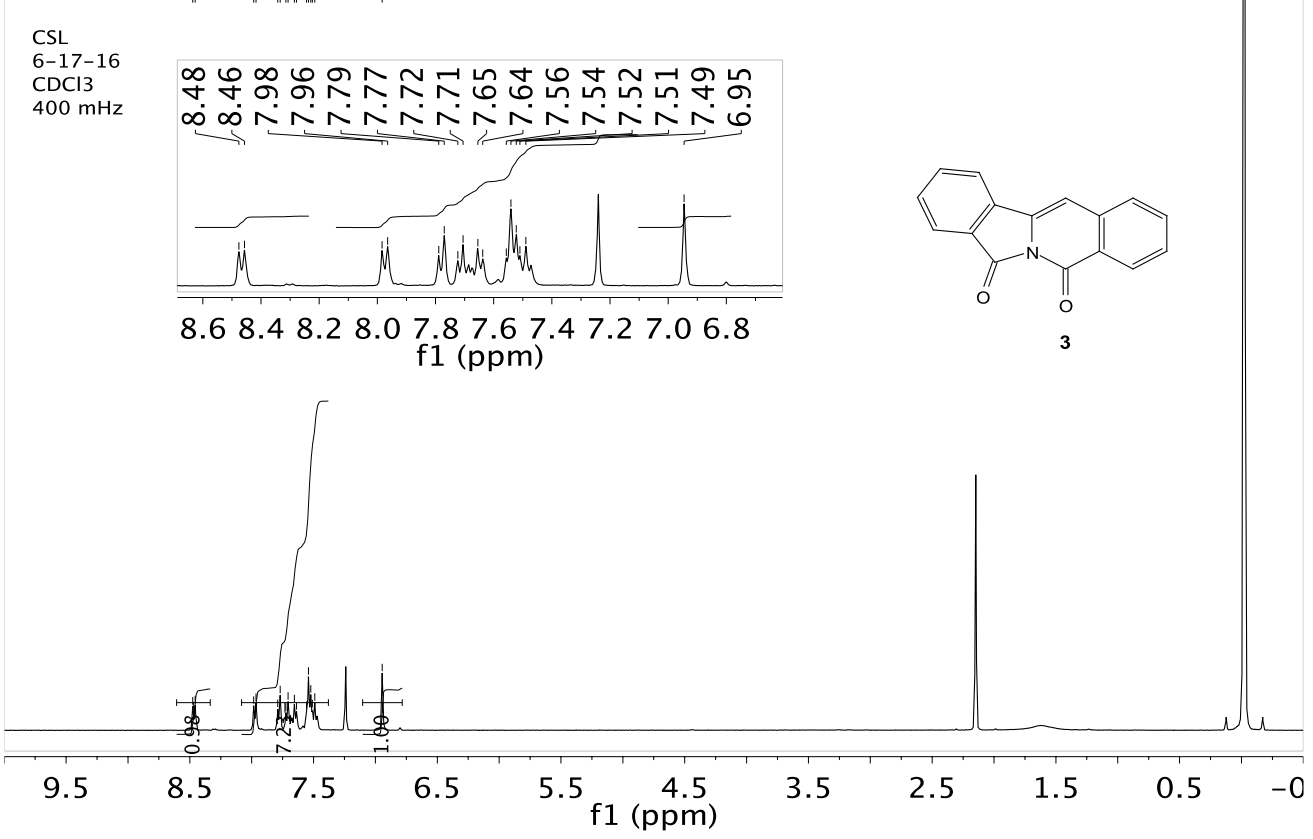

${ }^{1} \mathrm{H}$ NMR spectrum (400 MHz) of 3 in $\mathrm{CDCl}_{3}$

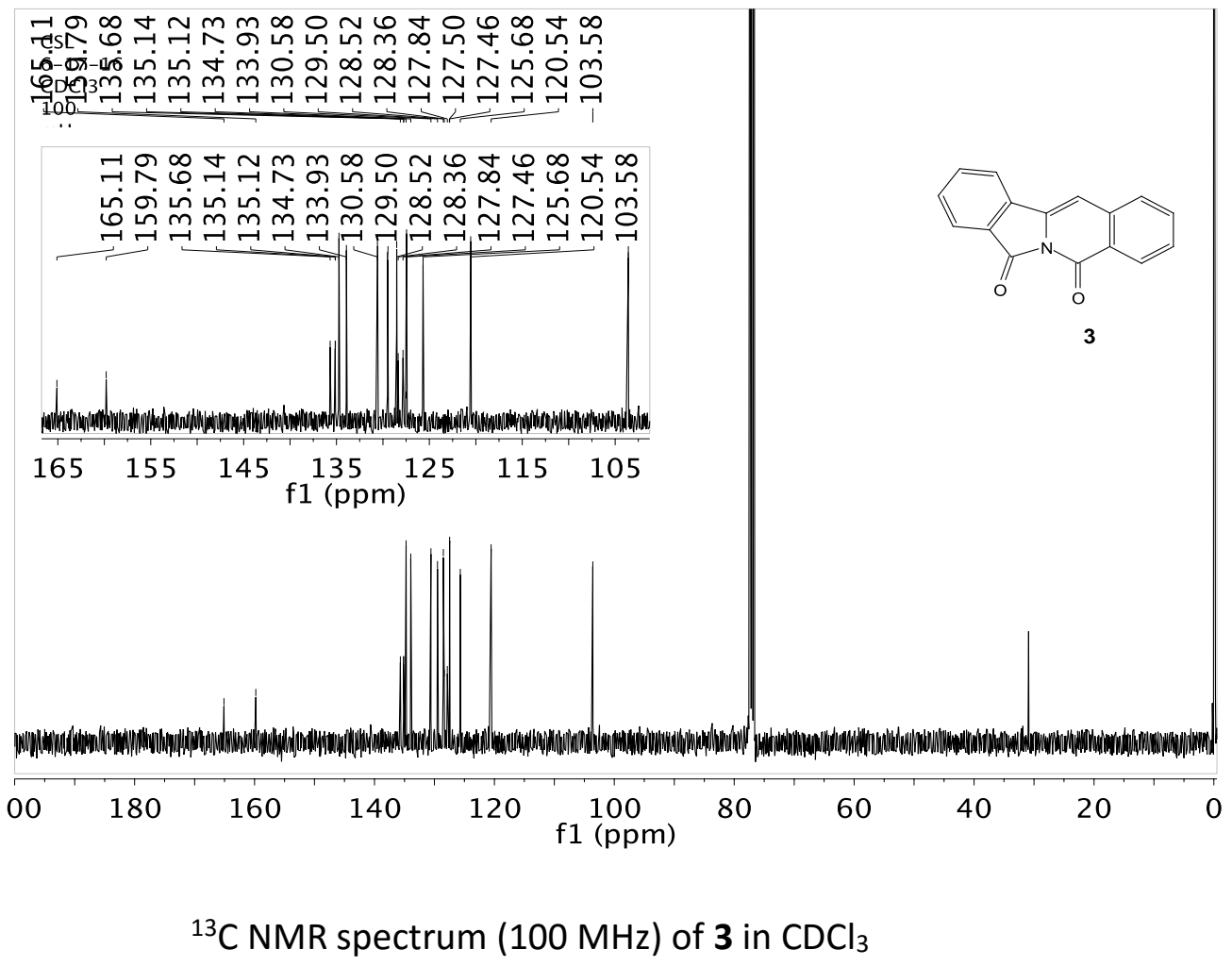




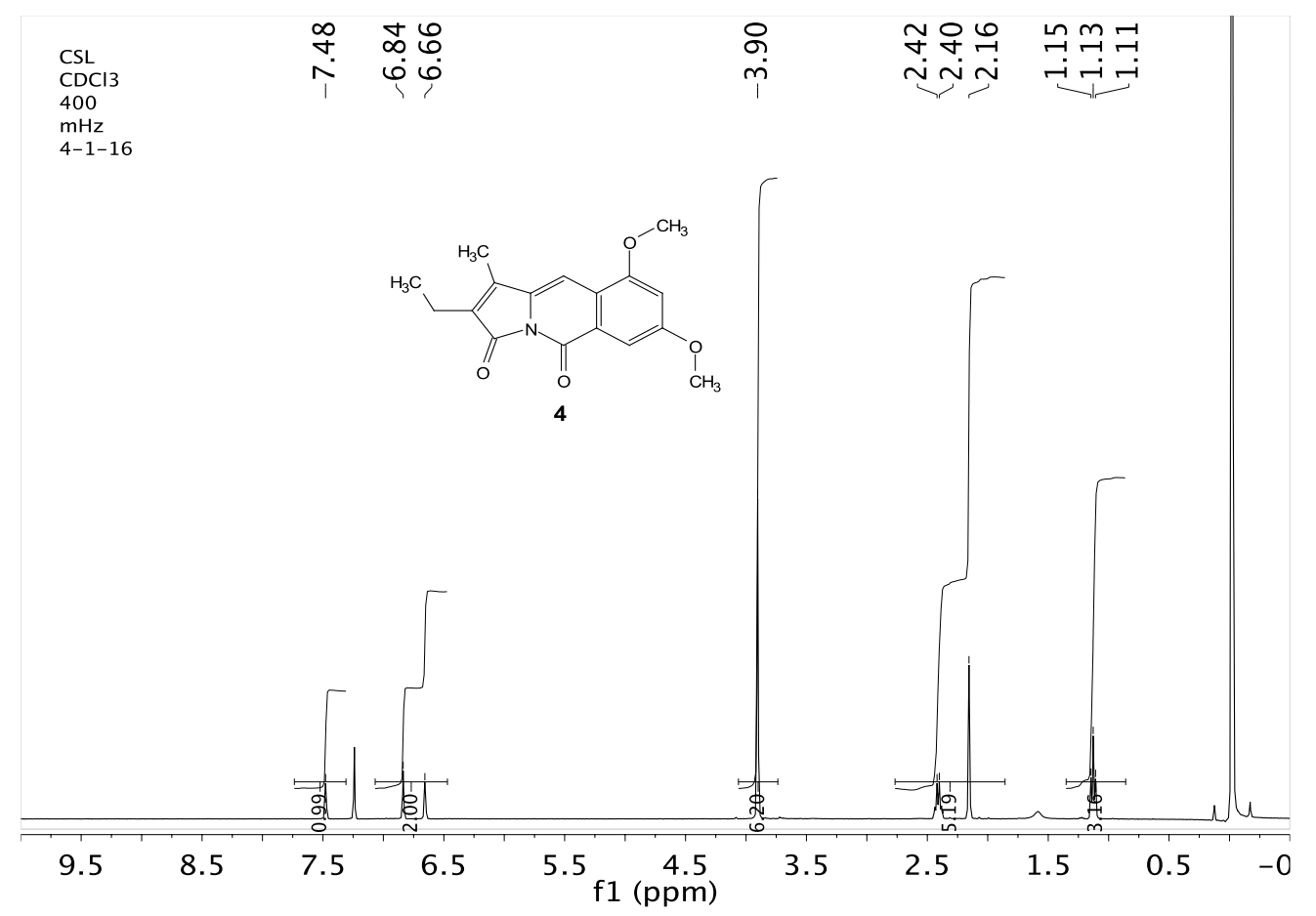

${ }^{1} \mathrm{H}$ NMR spectrum (400 MHz) of 4 in $\mathrm{CDCl}_{3}$

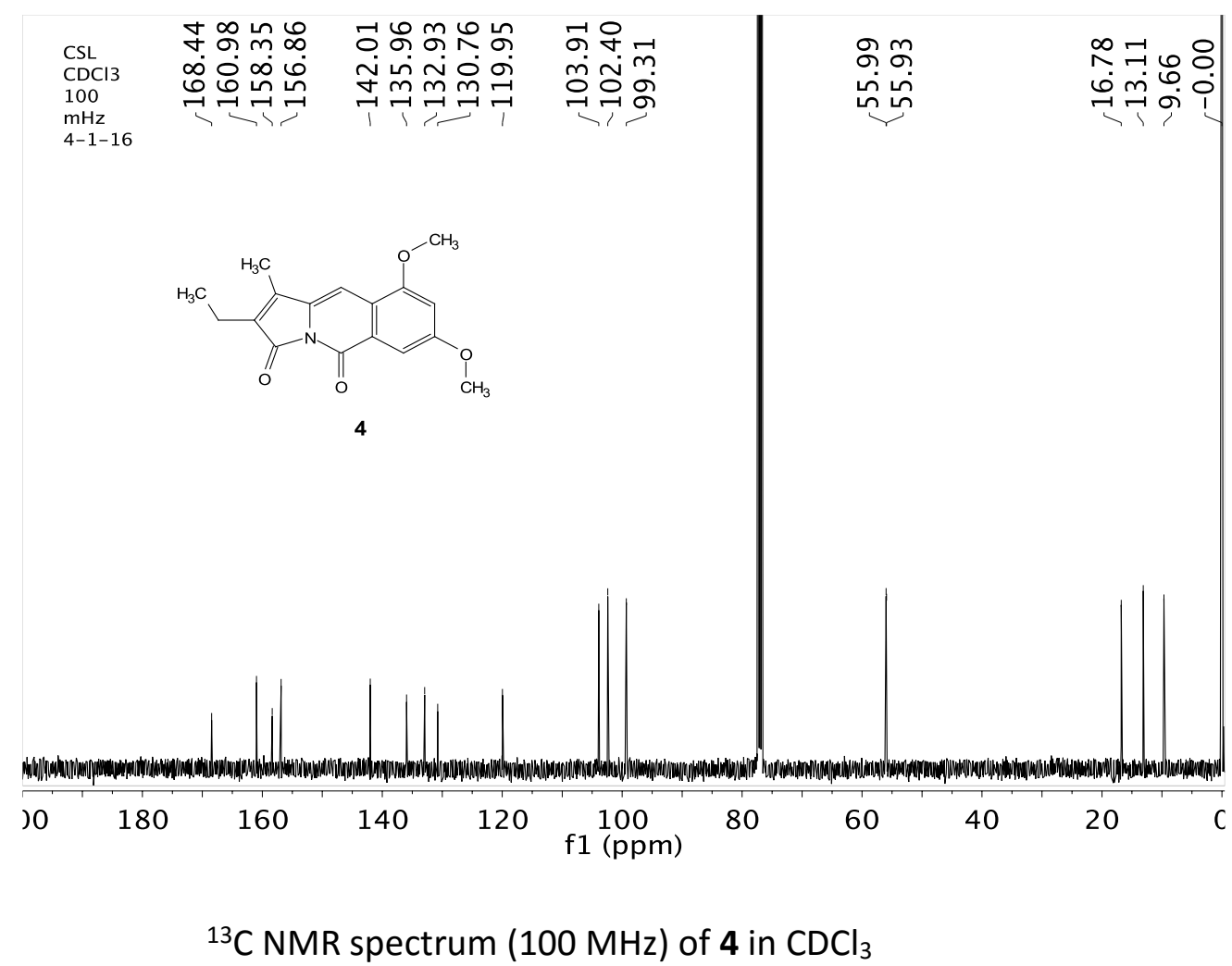




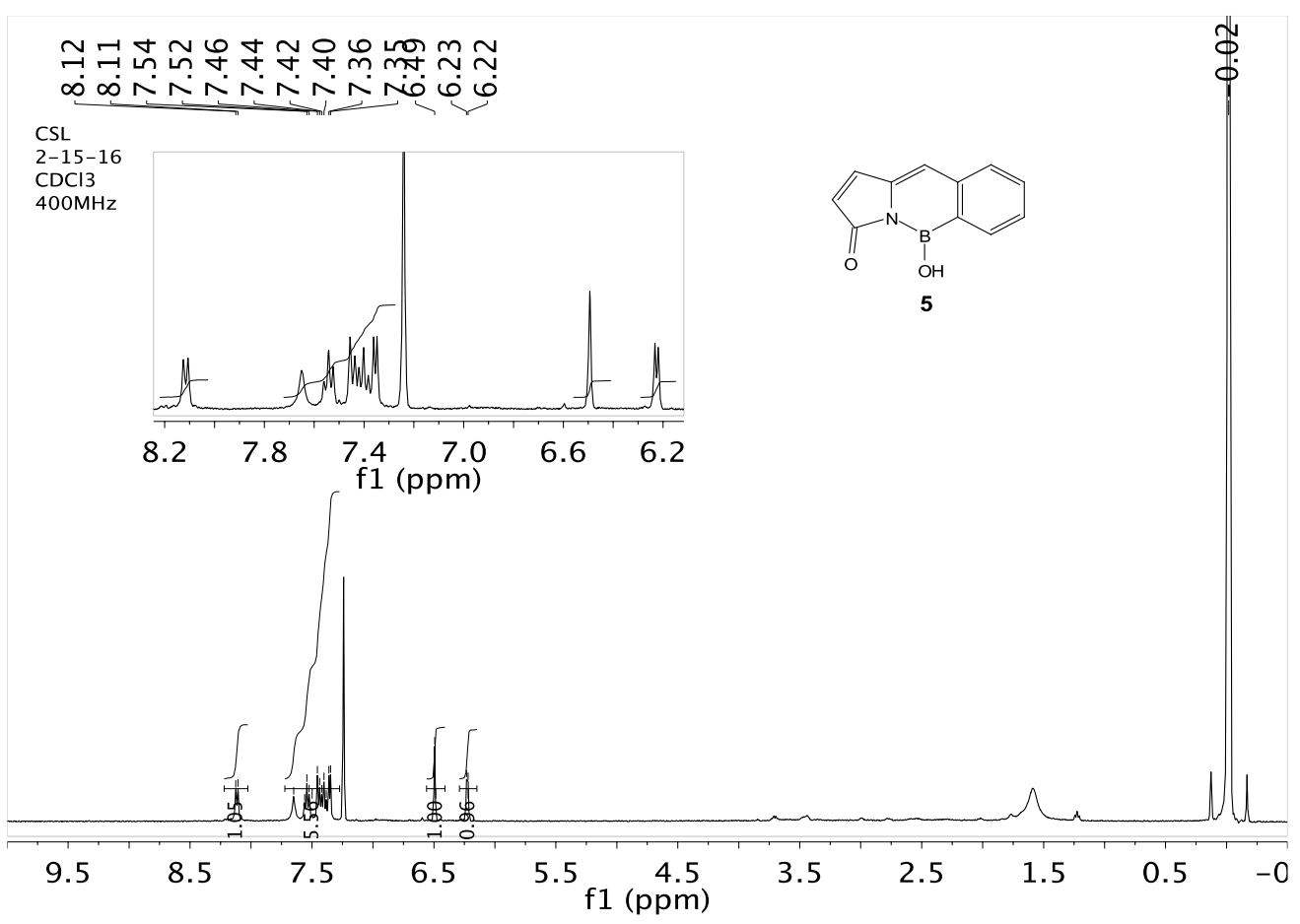

${ }^{1} \mathrm{H}$ NMR spectrum (400 MHz) of 5 in $\mathrm{CDCl}_{3}$

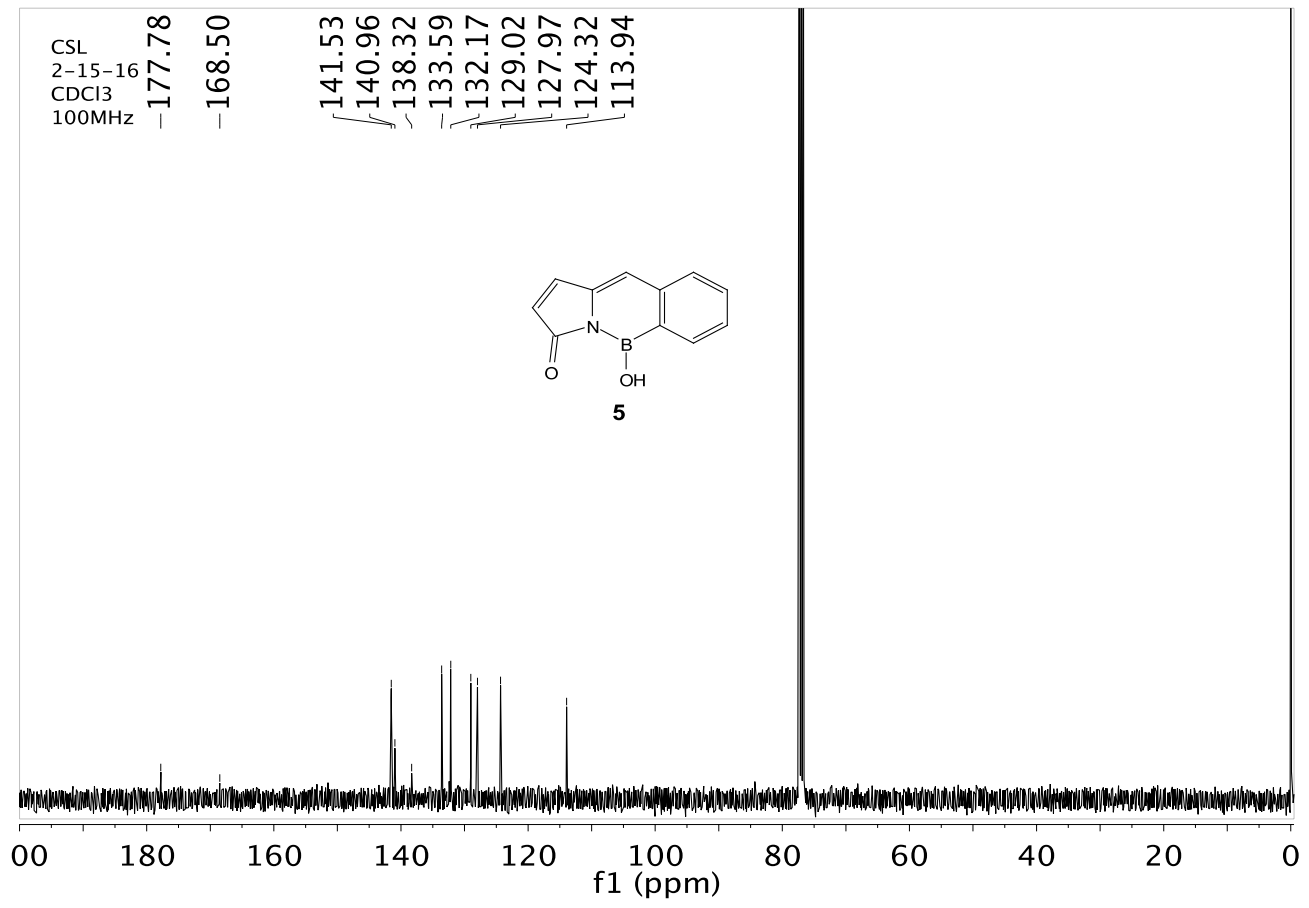

${ }^{13} \mathrm{C}$ NMR spectrum (100 MHz) of 5 in $\mathrm{CDCl}_{3}$ 


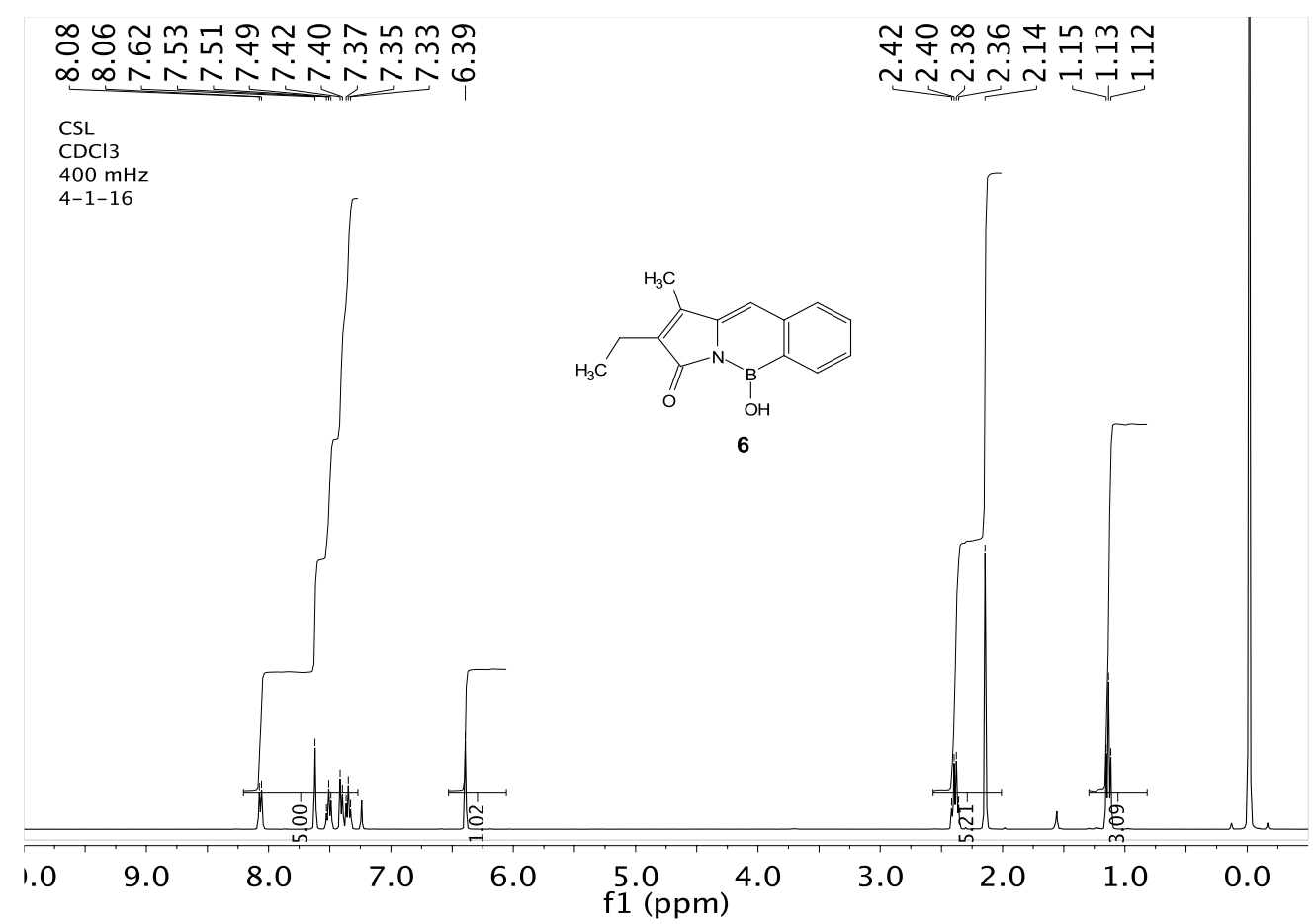

${ }^{1} \mathrm{H}$ NMR spectrum (400 MHz) of 6 in $\mathrm{CDCl}_{3}$

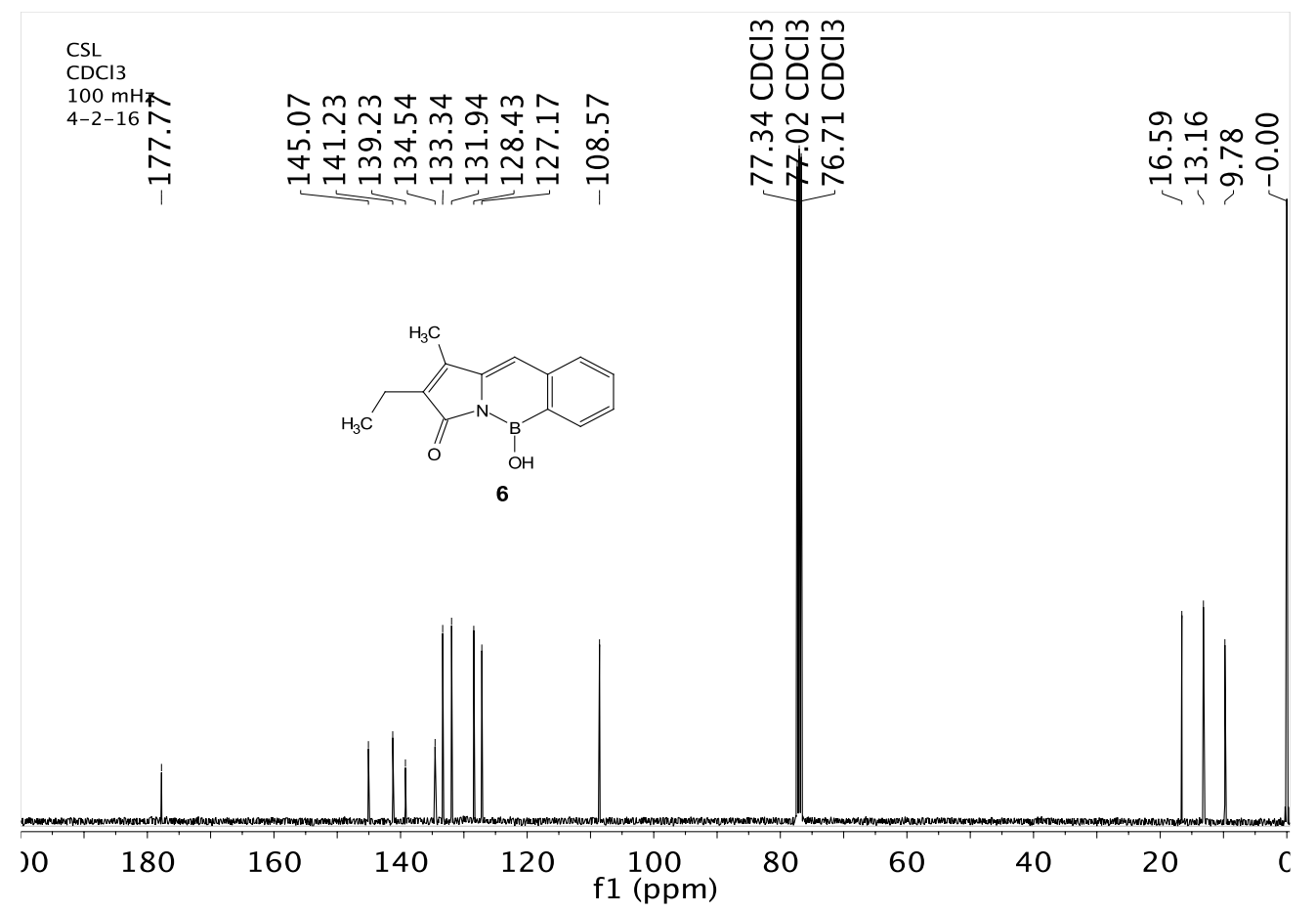

${ }^{13} \mathrm{C}$ NMR spectrum (100 MHz) of 6 in $\mathrm{CDCl}_{3}$ 


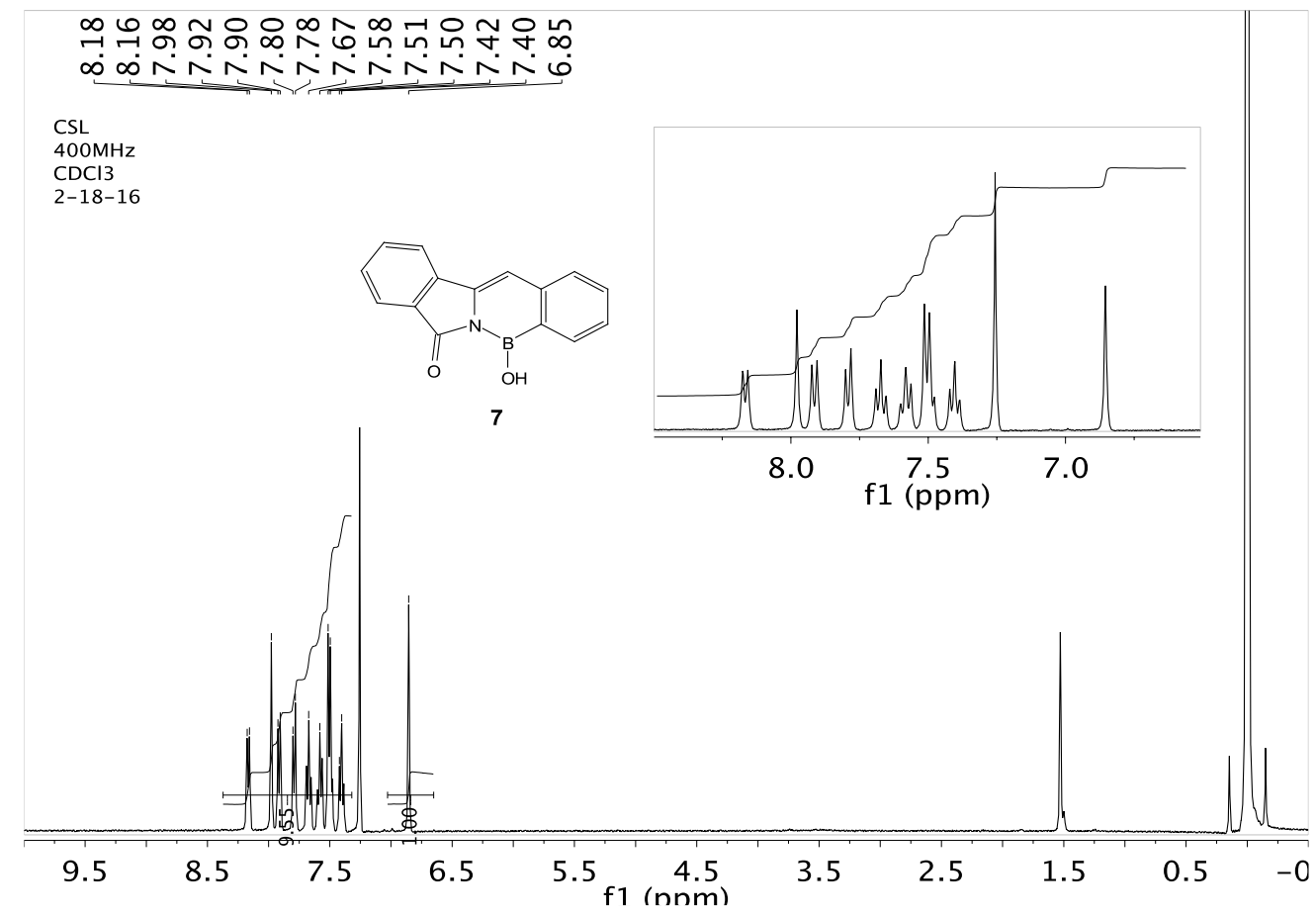

${ }^{1} \mathrm{H}$ NMR spectrum (400 MHz) of 7 in $\mathrm{CDCl}_{3}$

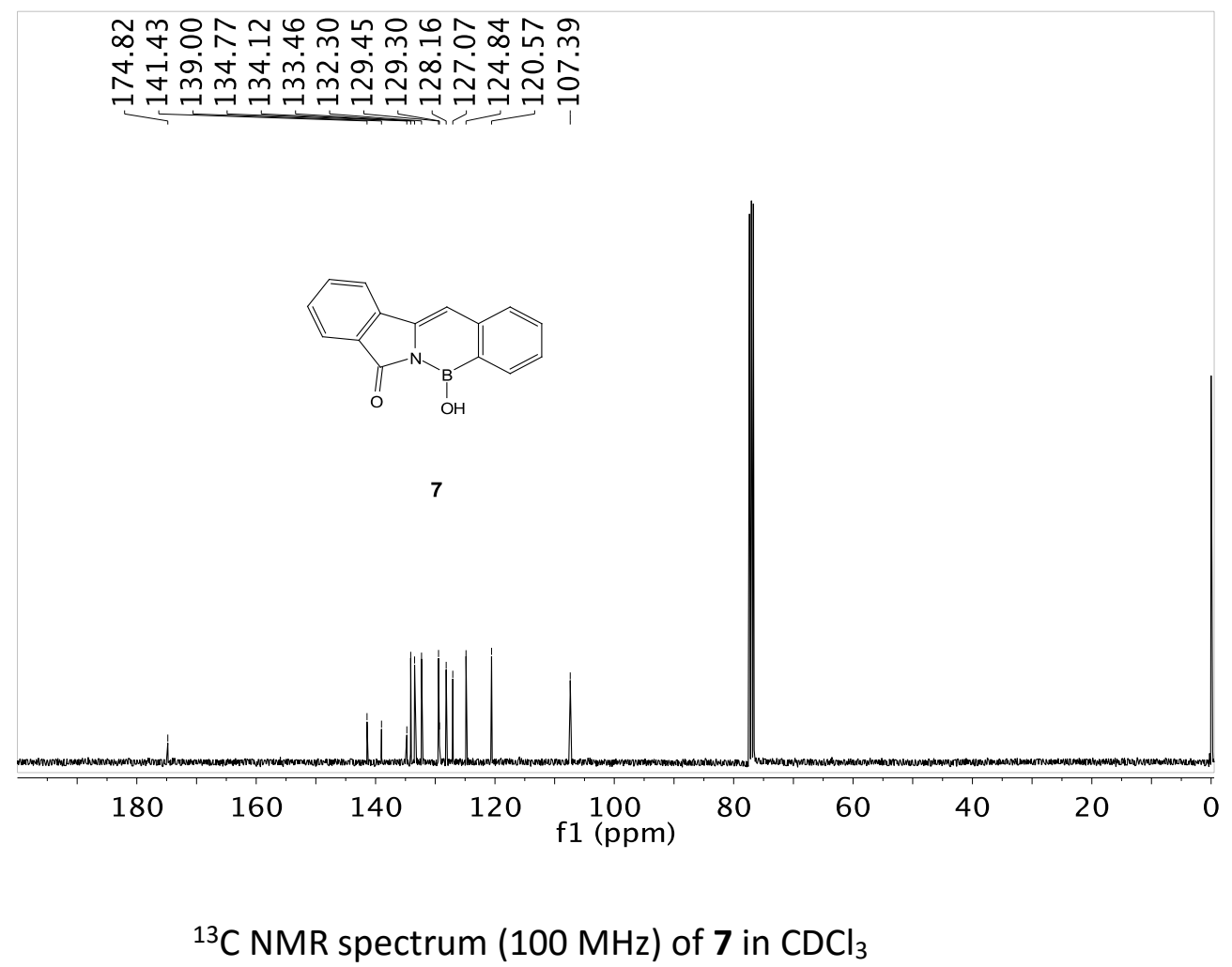




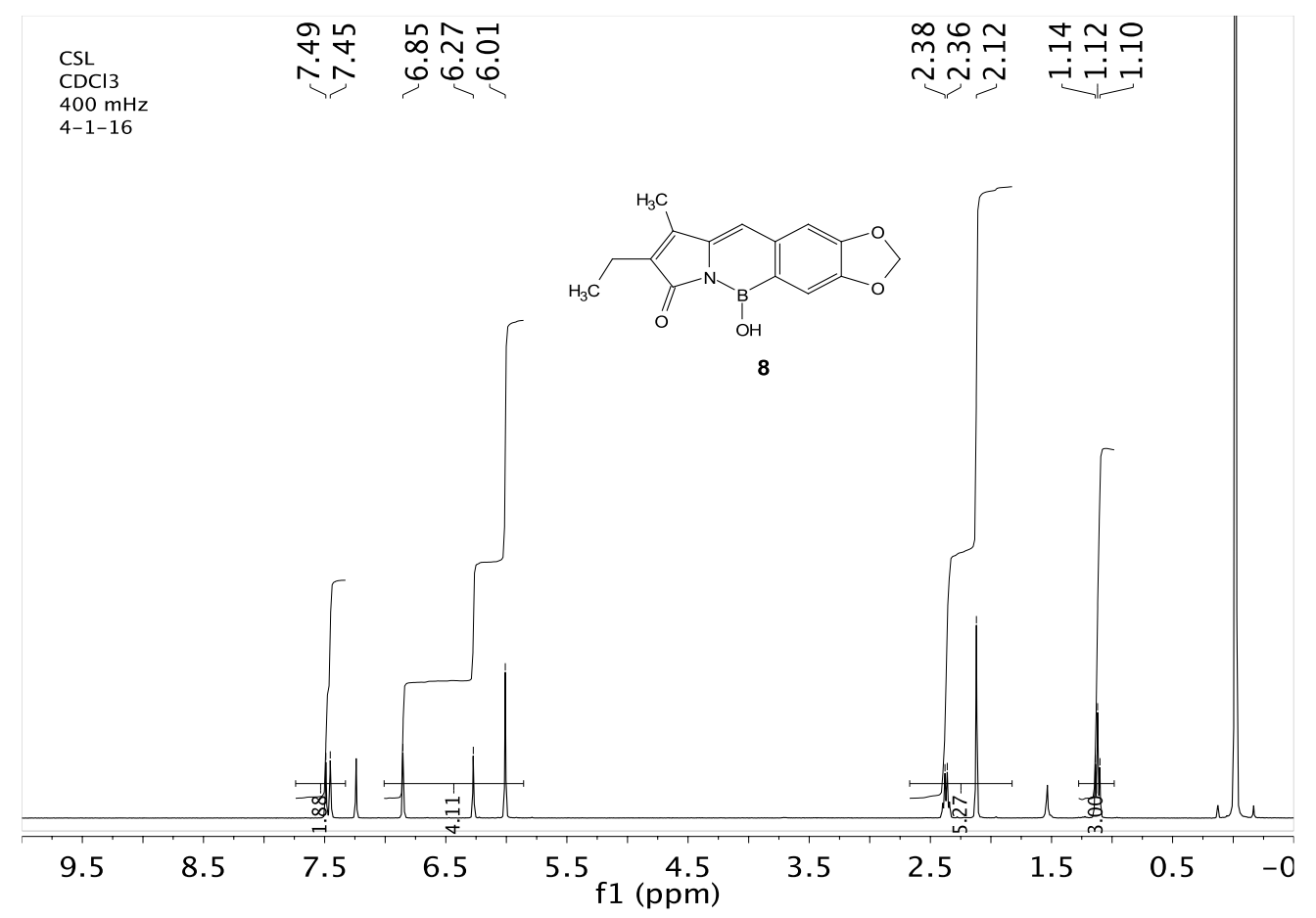

${ }^{1} \mathrm{H}$ NMR spectrum (400 MHz) of 8 in $\mathrm{CDCl}_{3}$

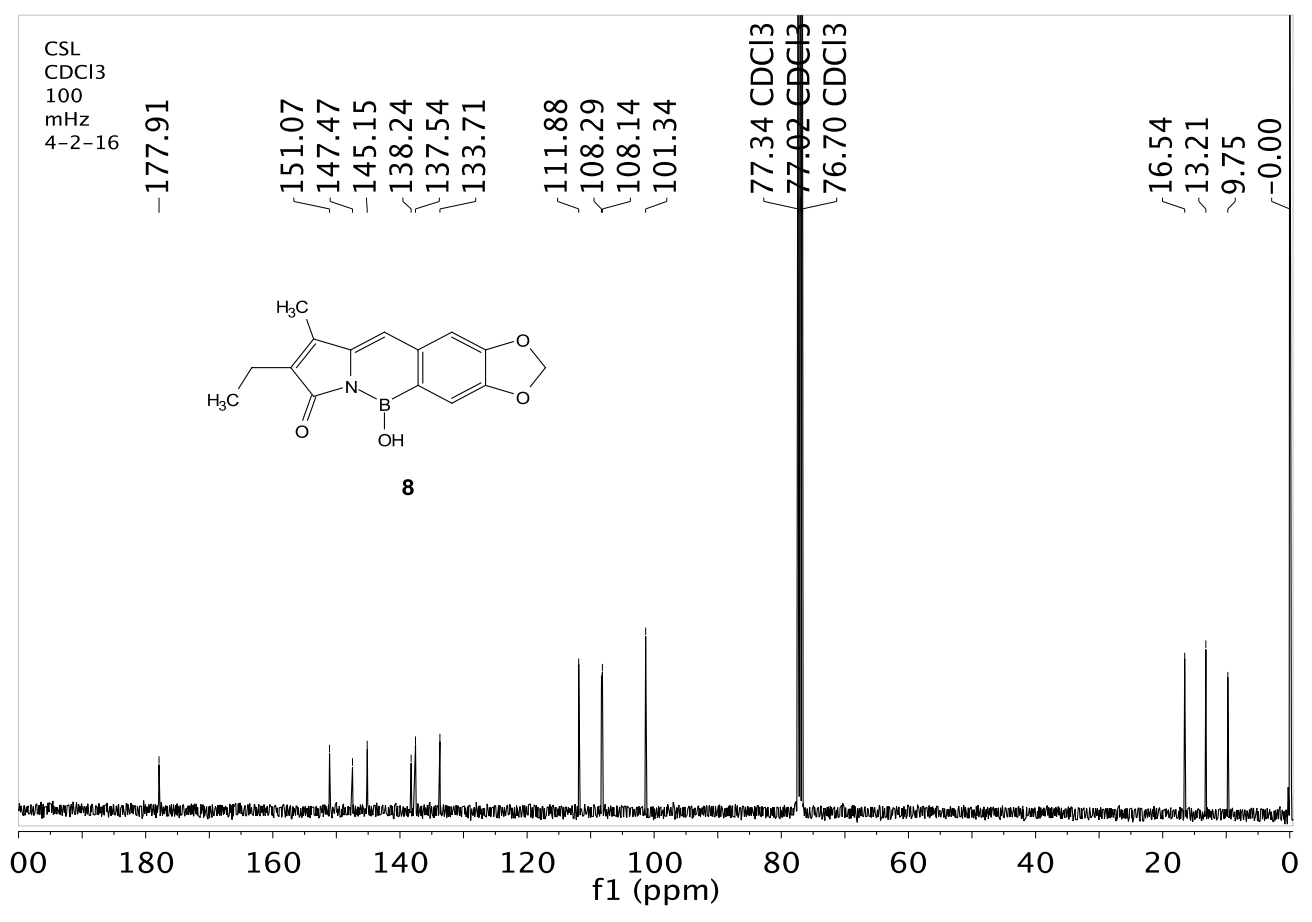

${ }^{13} \mathrm{C}$ NMR spectrum (100 MHz) of 8 in $\mathrm{CDCl}_{3}$ 


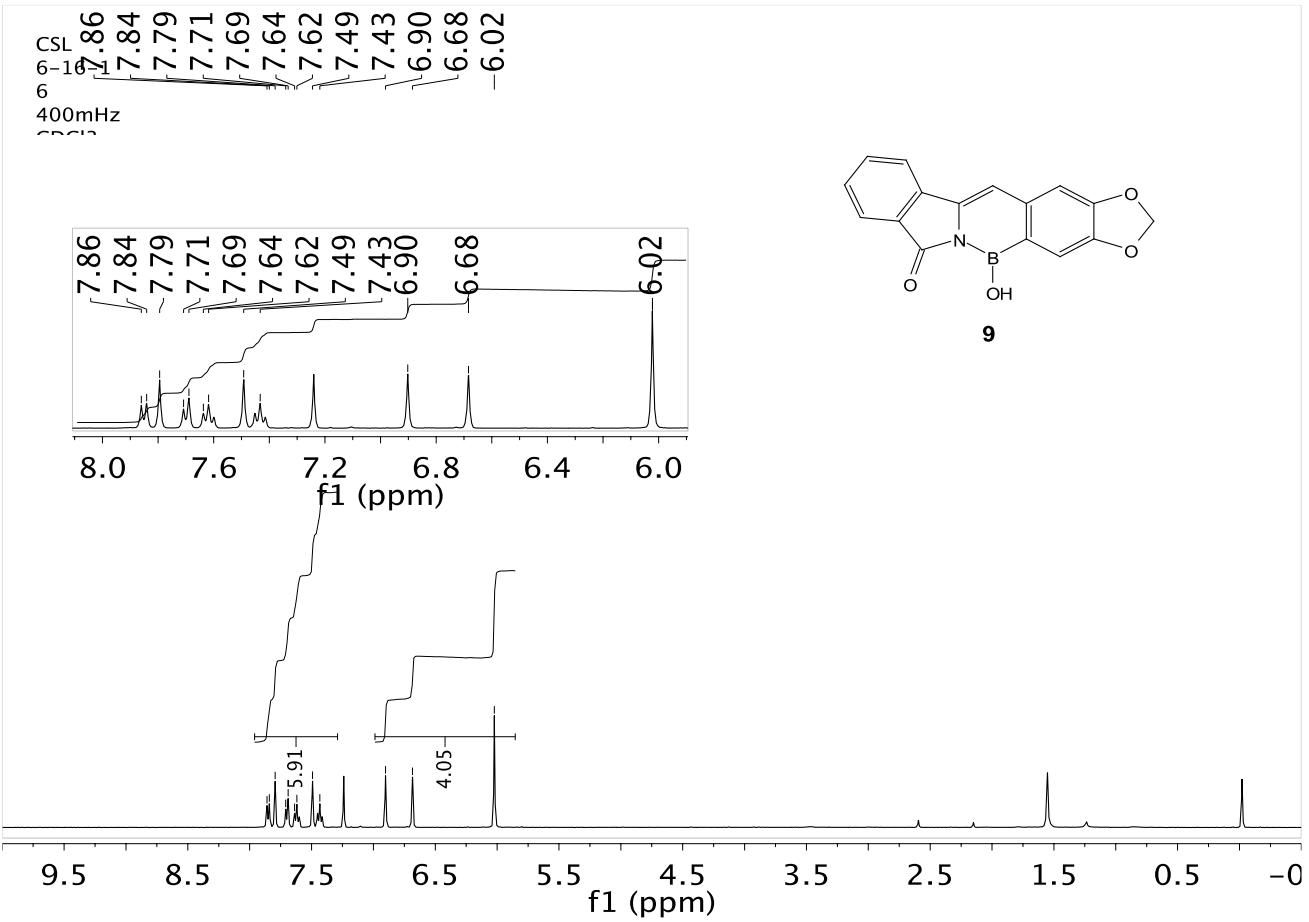

${ }^{1} \mathrm{H}$ NMR spectrum (400 MHz) of 9 in $\mathrm{CDCl}_{3}$

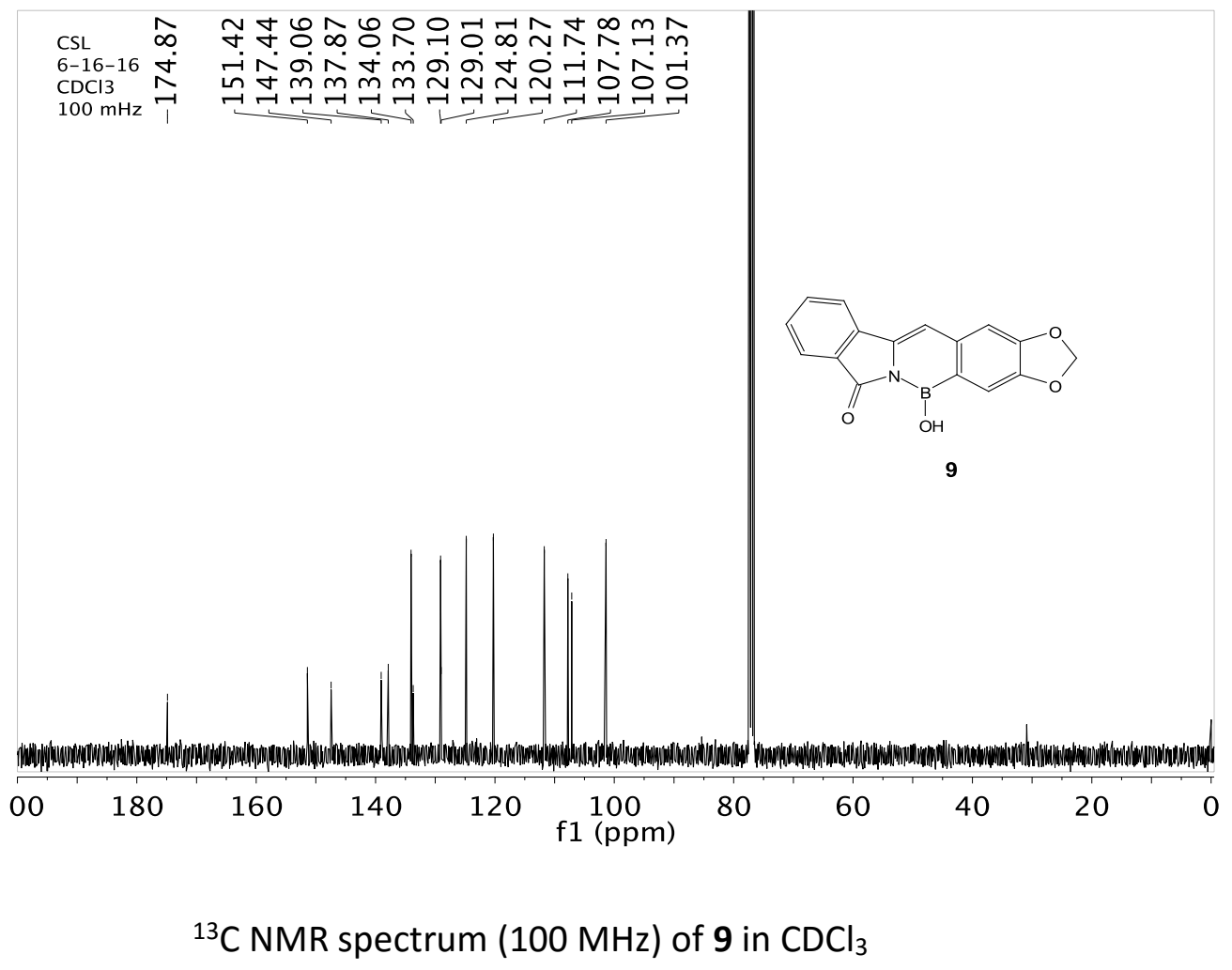




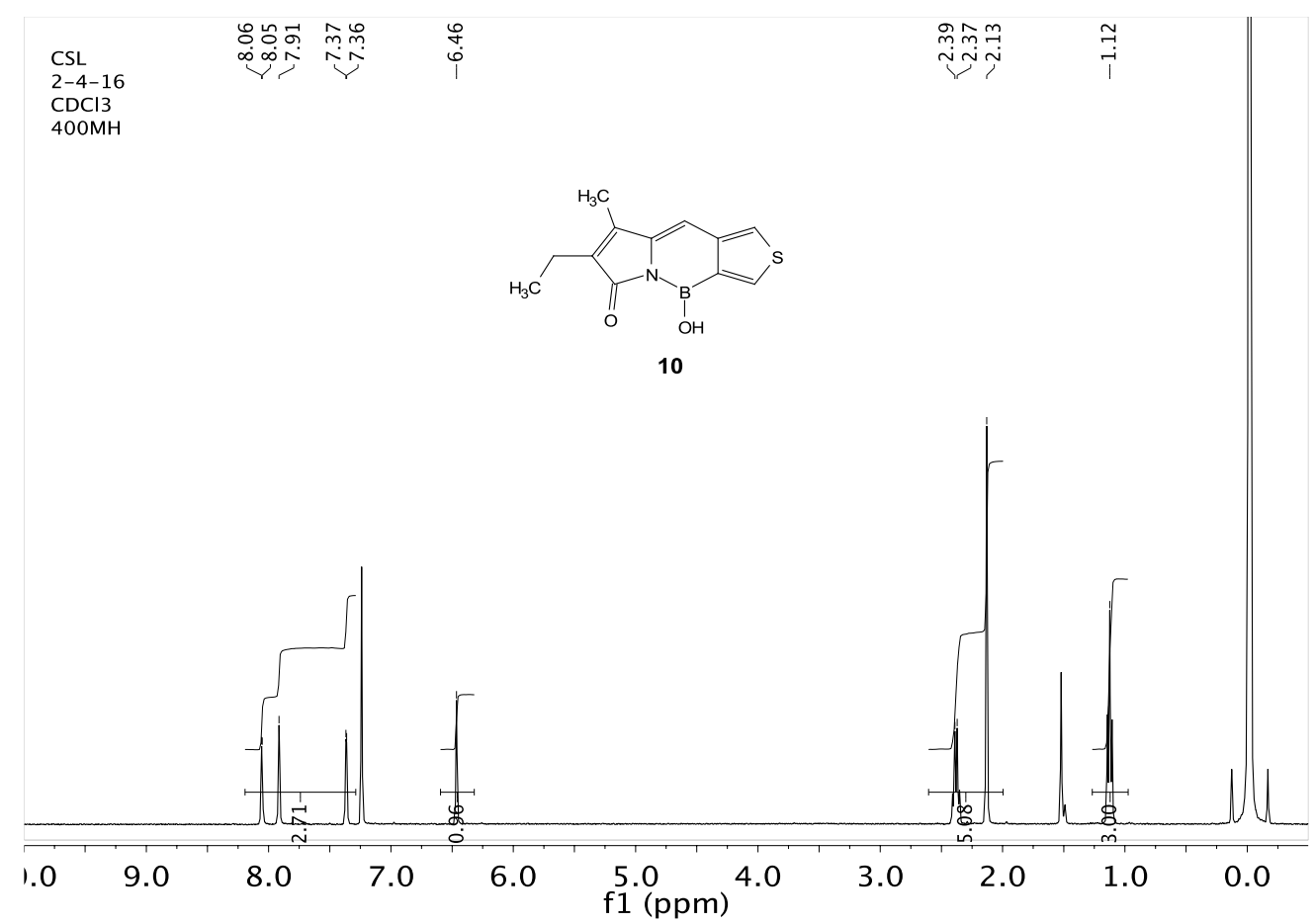

${ }^{1} \mathrm{H}$ NMR spectrum (400 MHz) of 10 in $\mathrm{CDCl}_{3}$

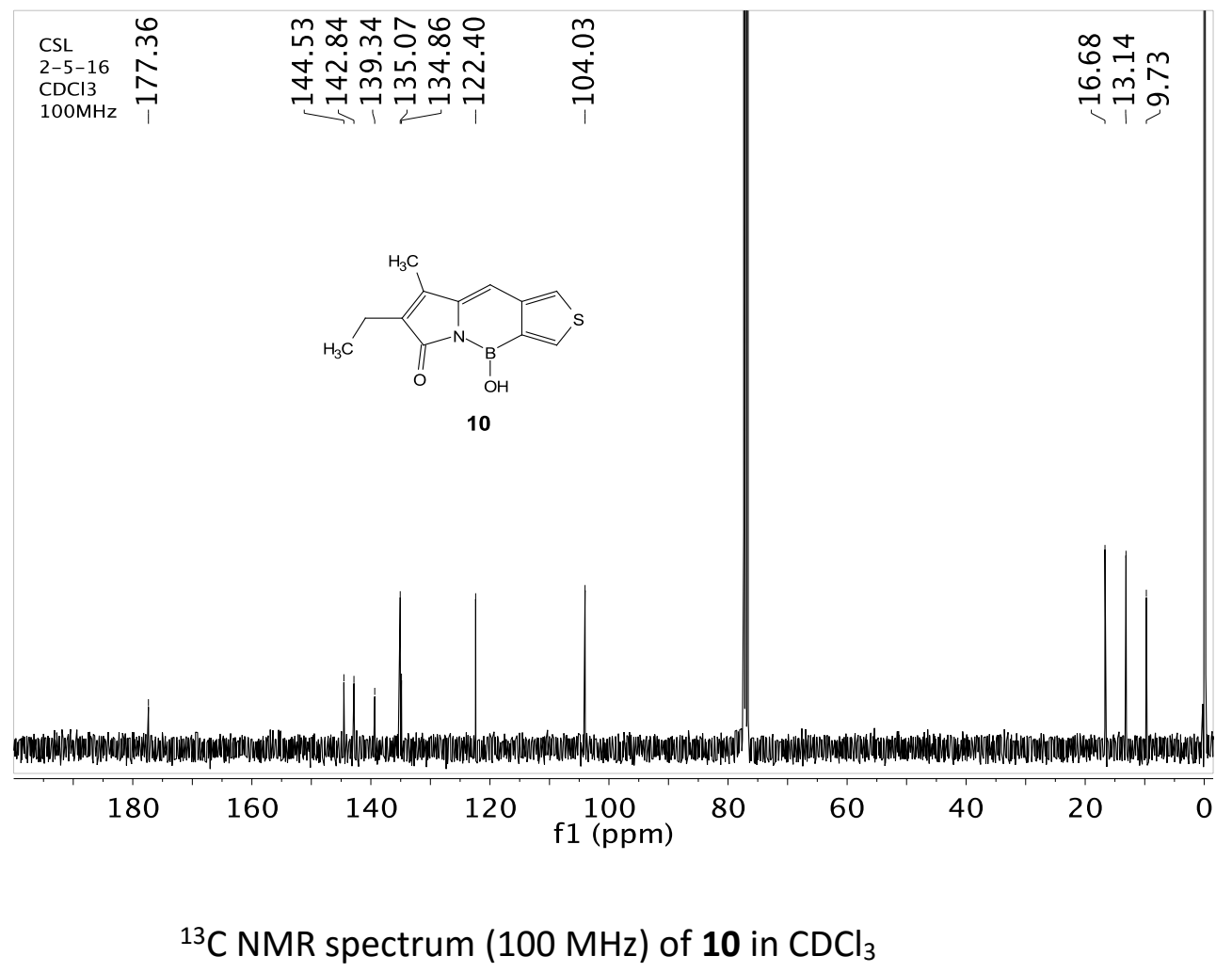




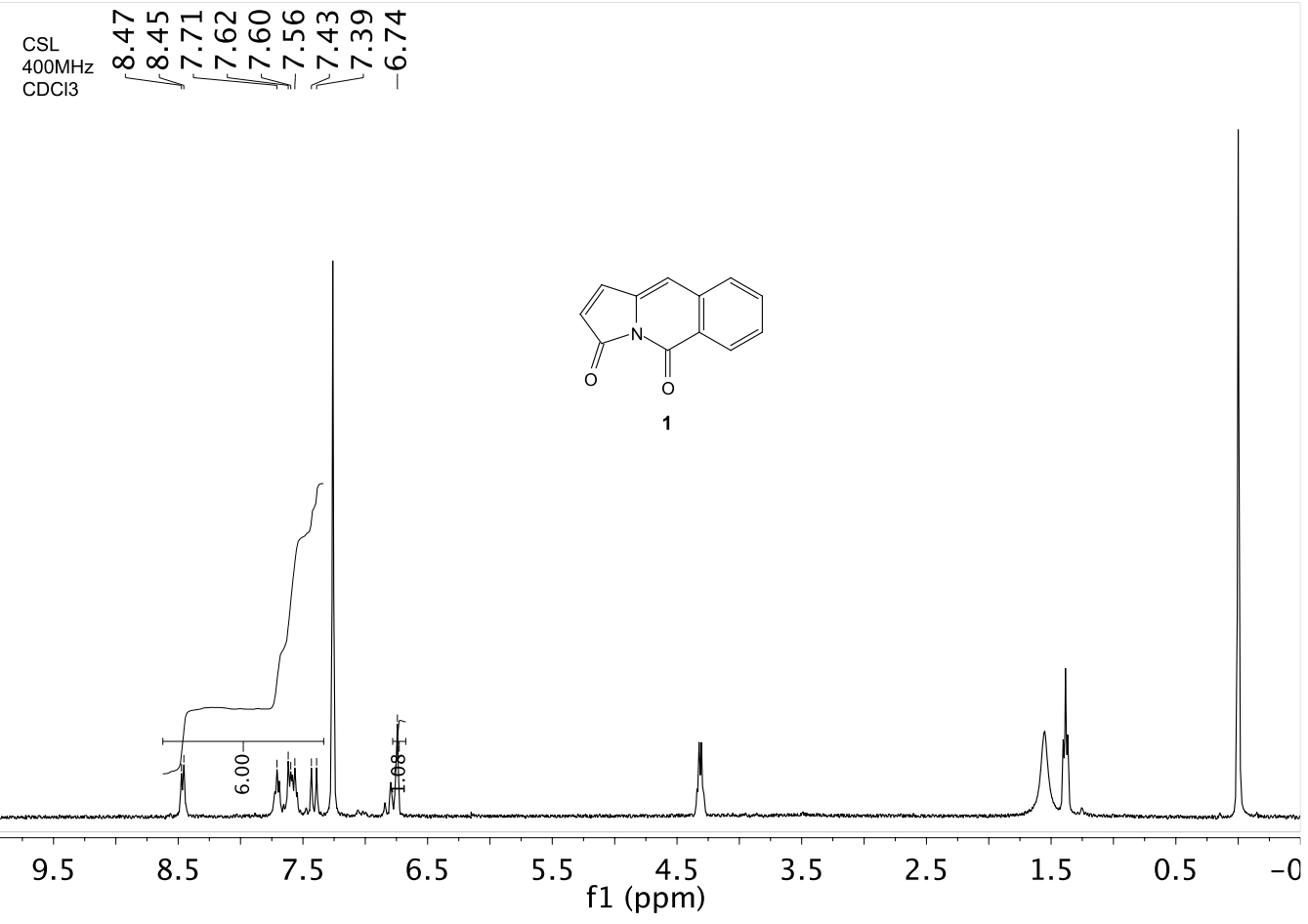

${ }^{1} \mathrm{H}$ NMR spectrum (400 MHz) of 1 in $\mathrm{CDCl}_{3}$

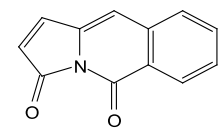

1

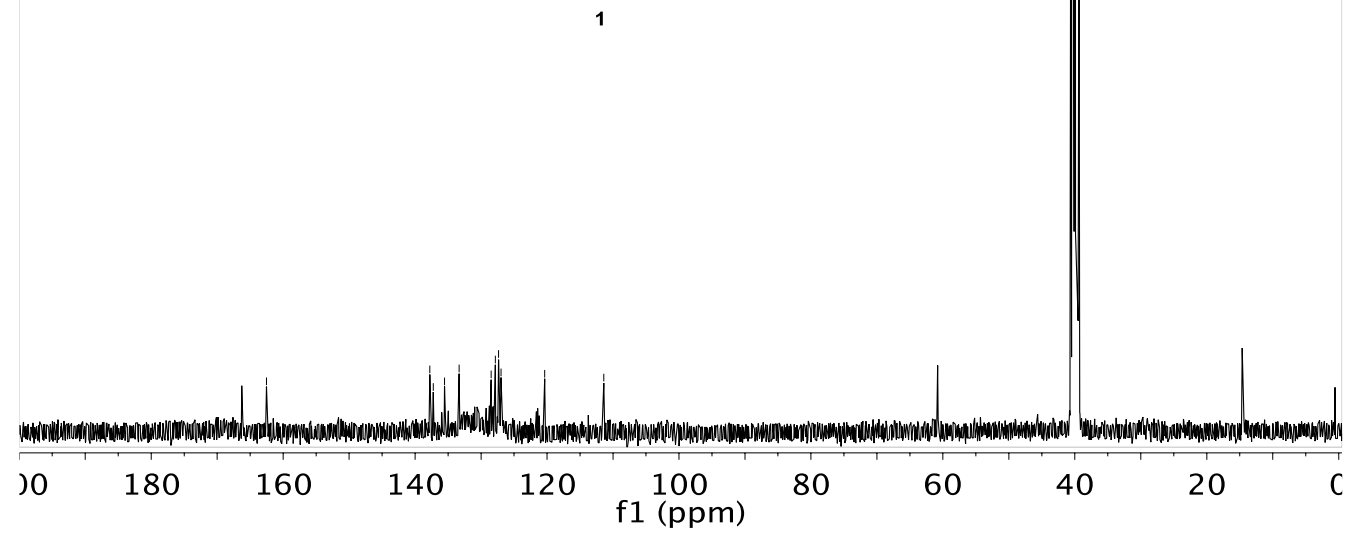

${ }^{13} \mathrm{C}$ NMR spectrum (100 MHz) of 1 in DMSO 


\section{${ }^{11}$ B NMR}

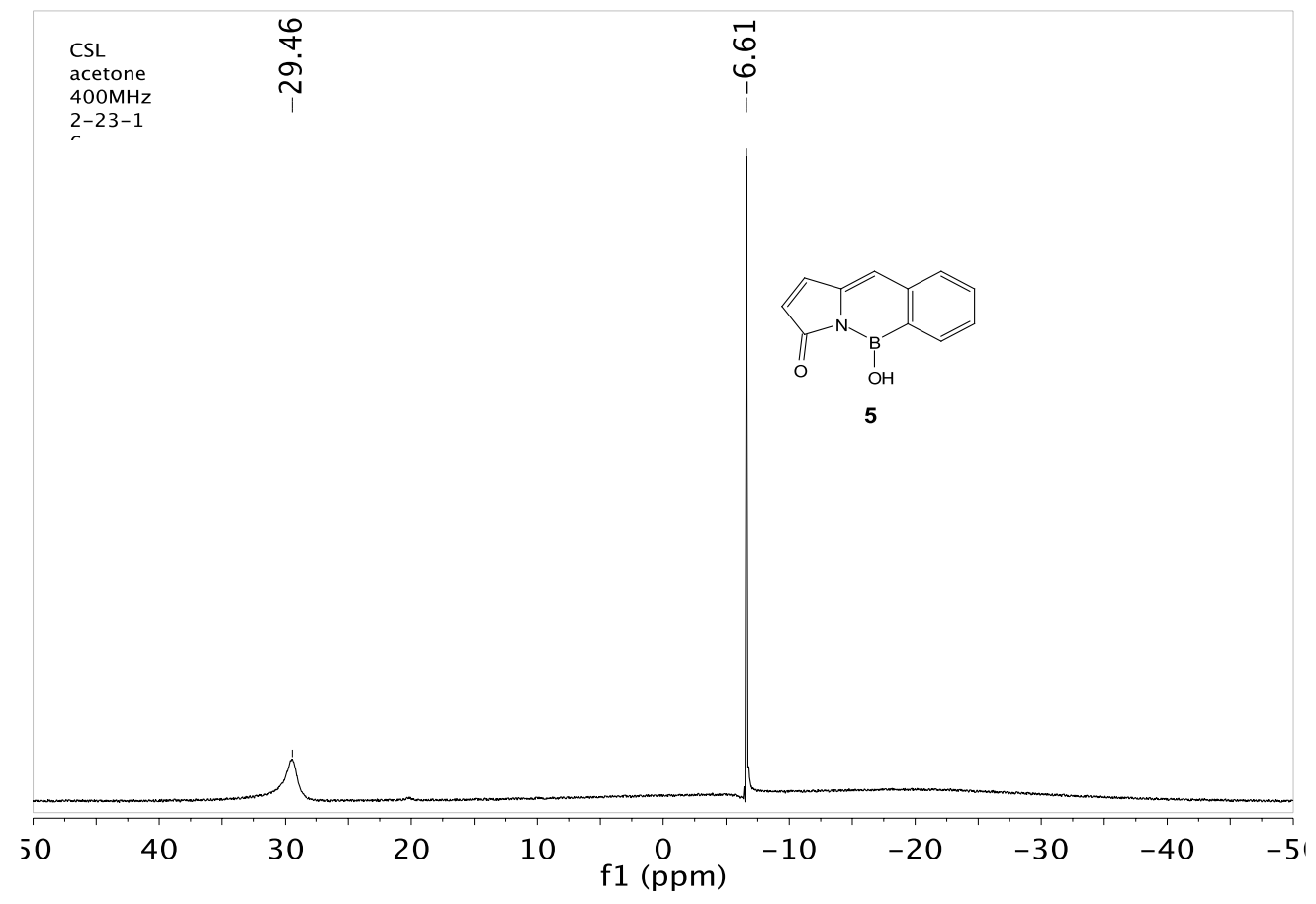

${ }^{11} \mathrm{~B}$ NMR spectrum (128 MHz) of 5 in $\mathrm{CDCl}_{3}$

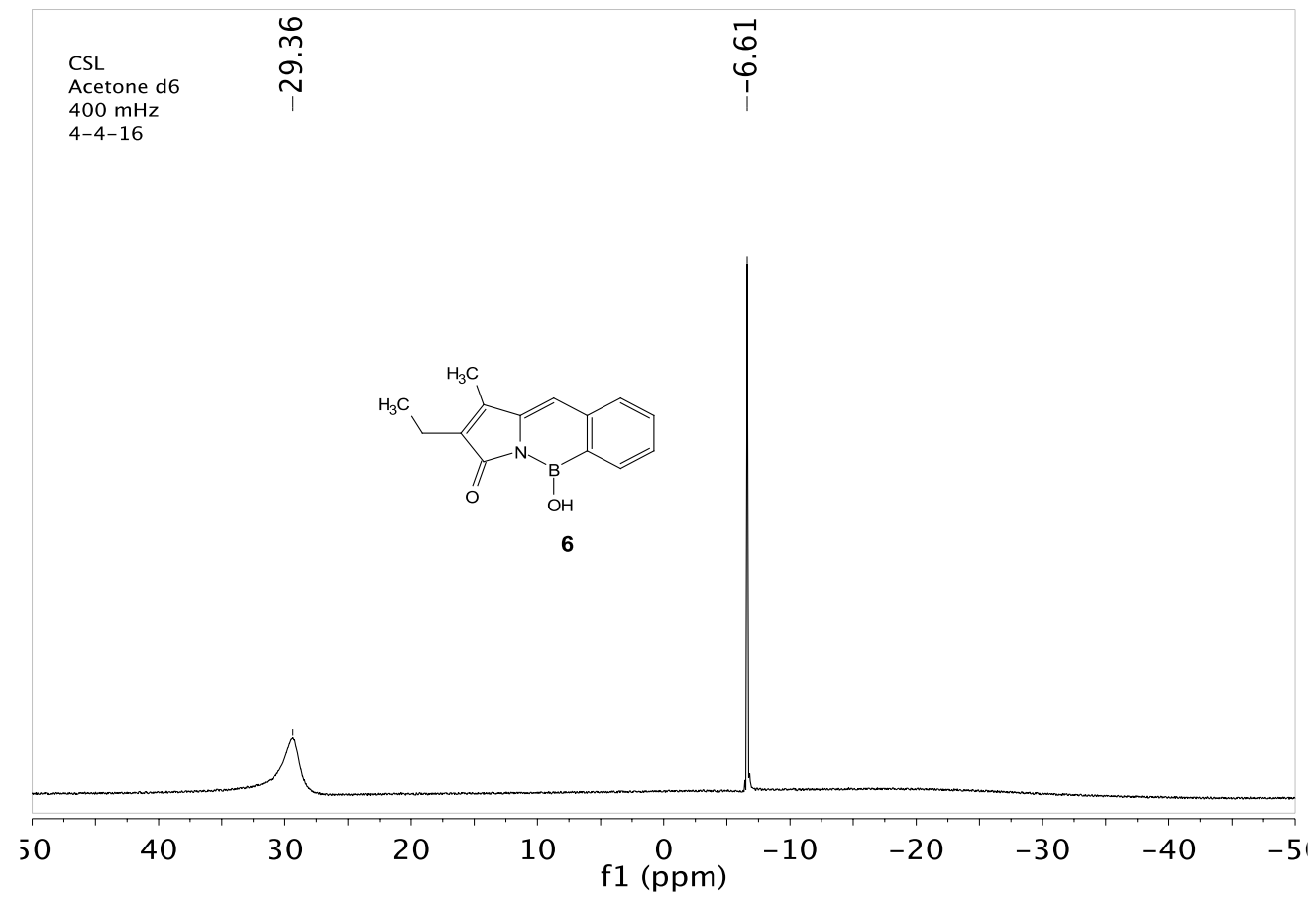

${ }^{11} \mathrm{~B} N M R$ spectrum $\left(128 \mathrm{MHz}\right.$ ) of 6 in $\mathrm{CDCl}_{3}$ 


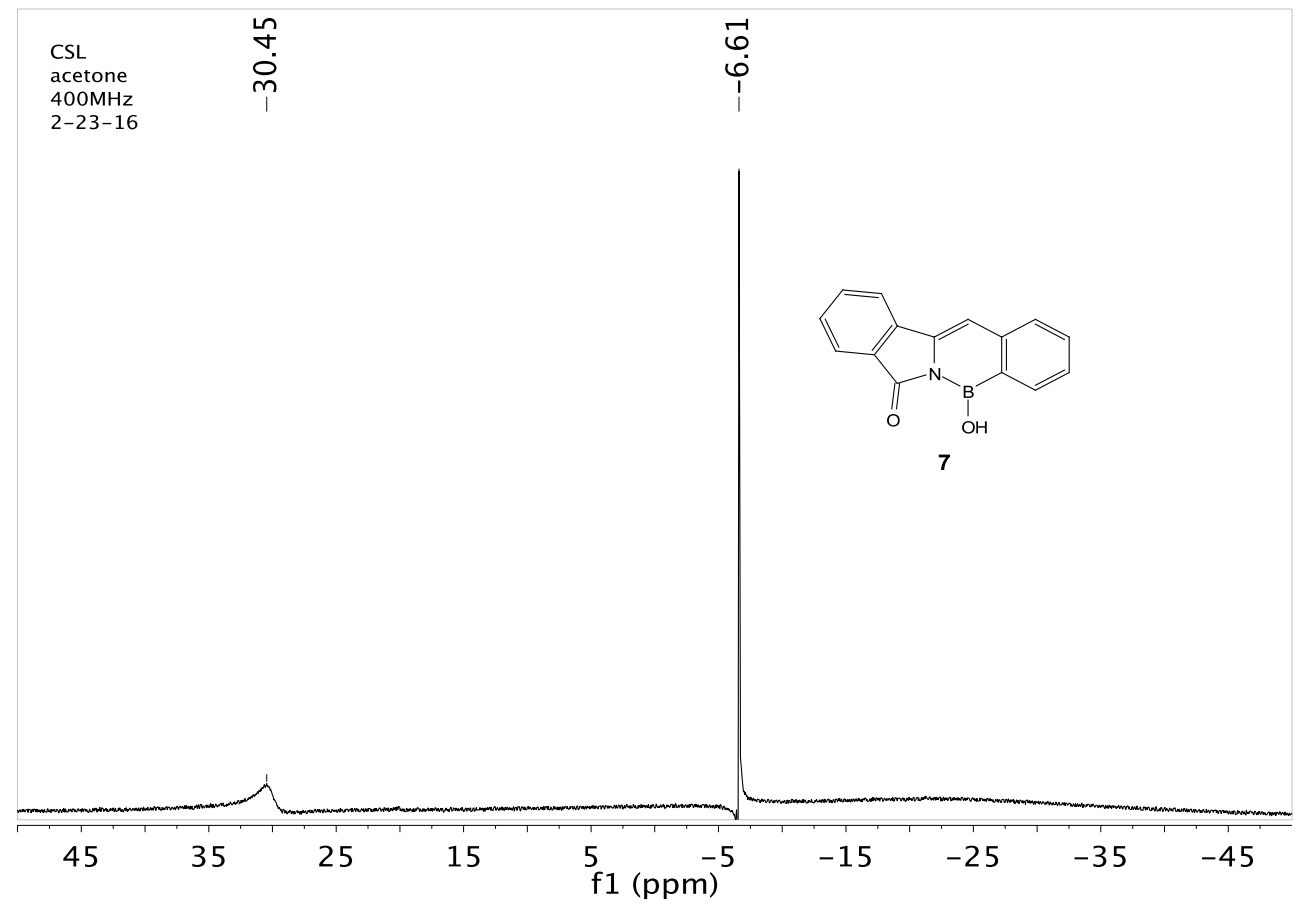

${ }^{11} \mathrm{~B}$ NMR spectrum $(128 \mathrm{MHz})$ of 7 in $\mathrm{CDCl}_{3}$

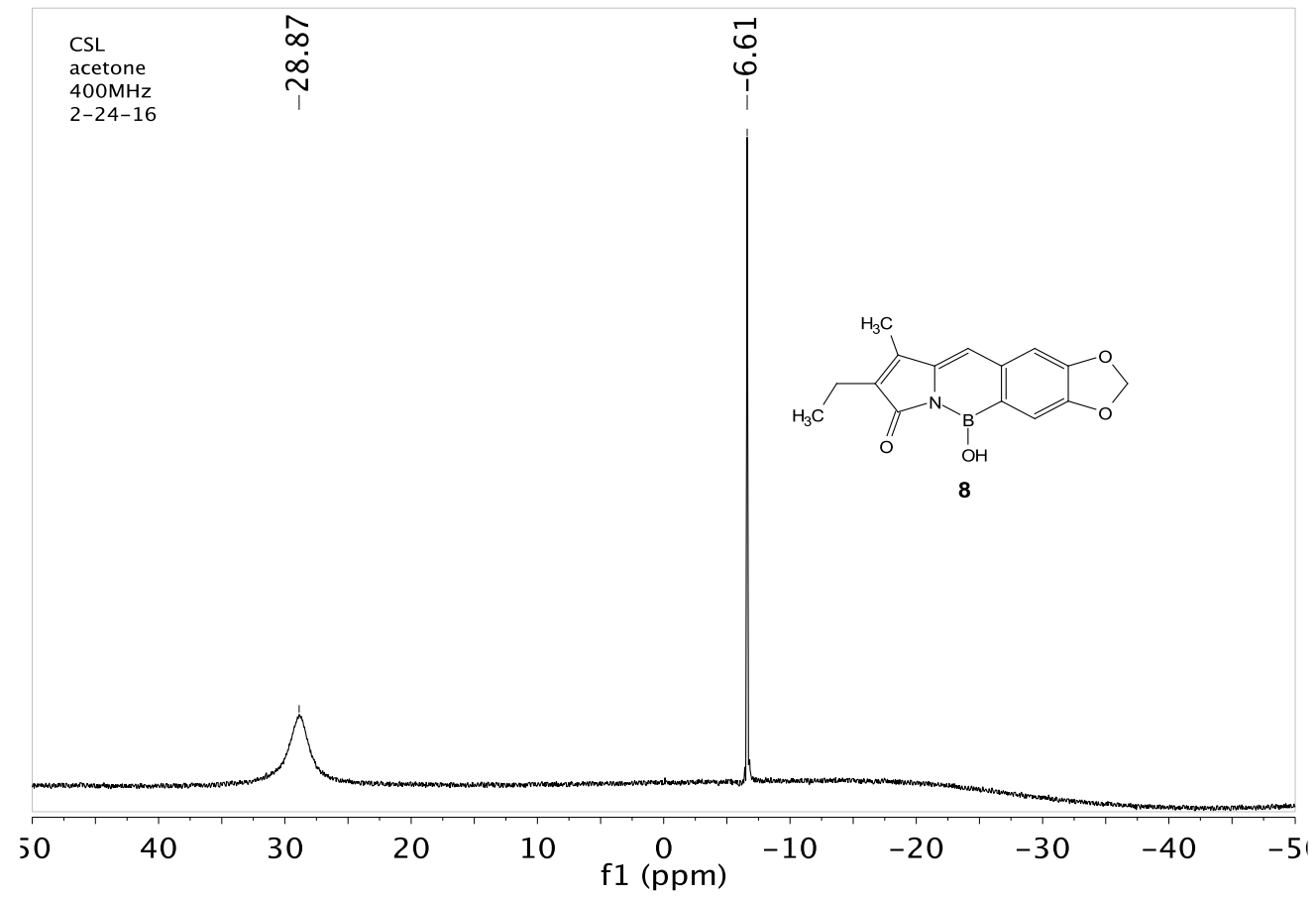

${ }^{11} \mathrm{~B}$ NMR spectrum (128 MHz) of 8 in $\mathrm{CDCl}_{3}$ 


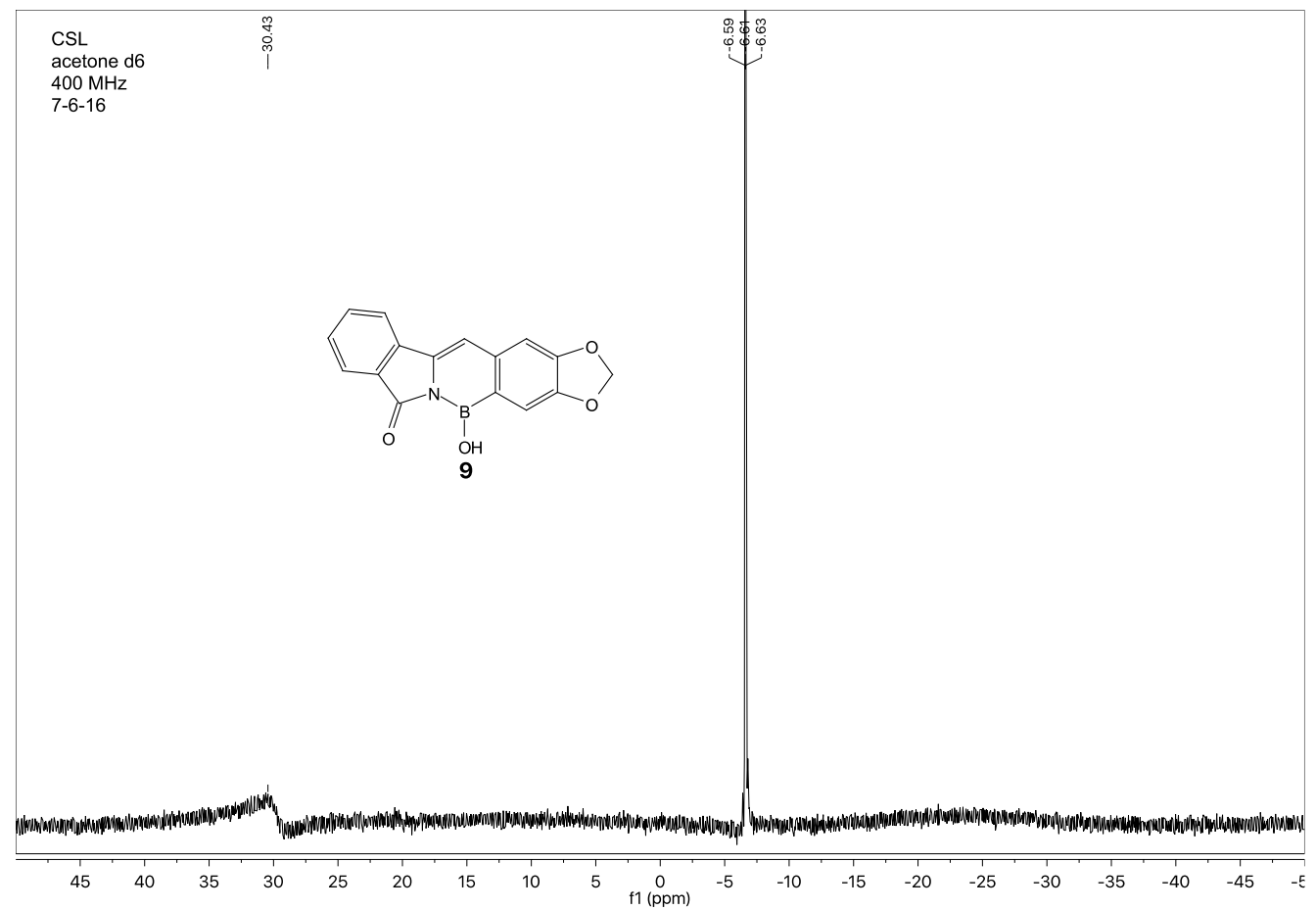

${ }^{11} \mathrm{~B}$ NMR spectrum (128 MHz) of 9 in $\mathrm{CDCl}_{3}$

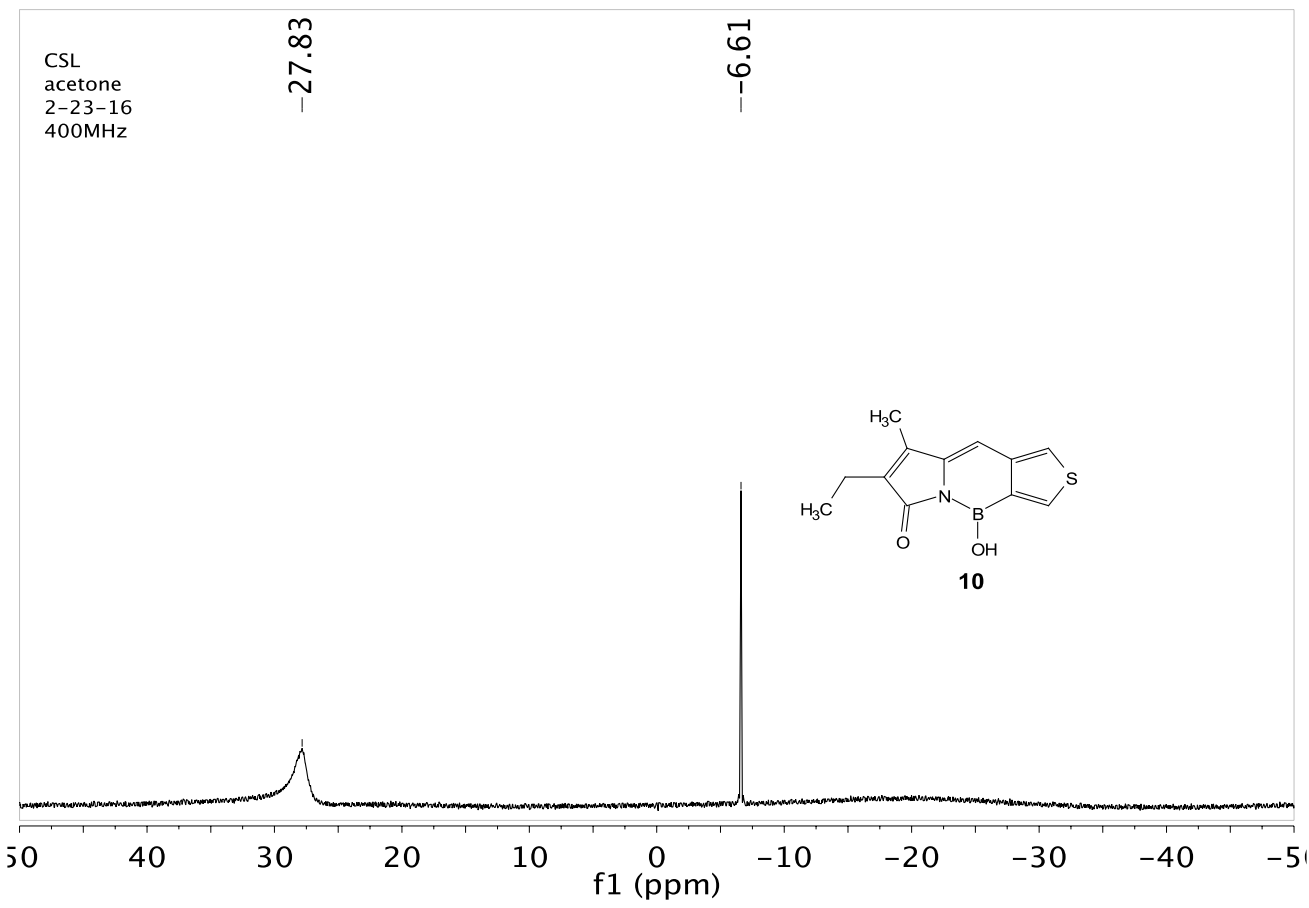

${ }^{11} \mathrm{~B}$ NMR spectrum $\left(128 \mathrm{MHz}\right.$ ) of 10 in $\mathrm{CDCl}_{3}$ 
FT-IR
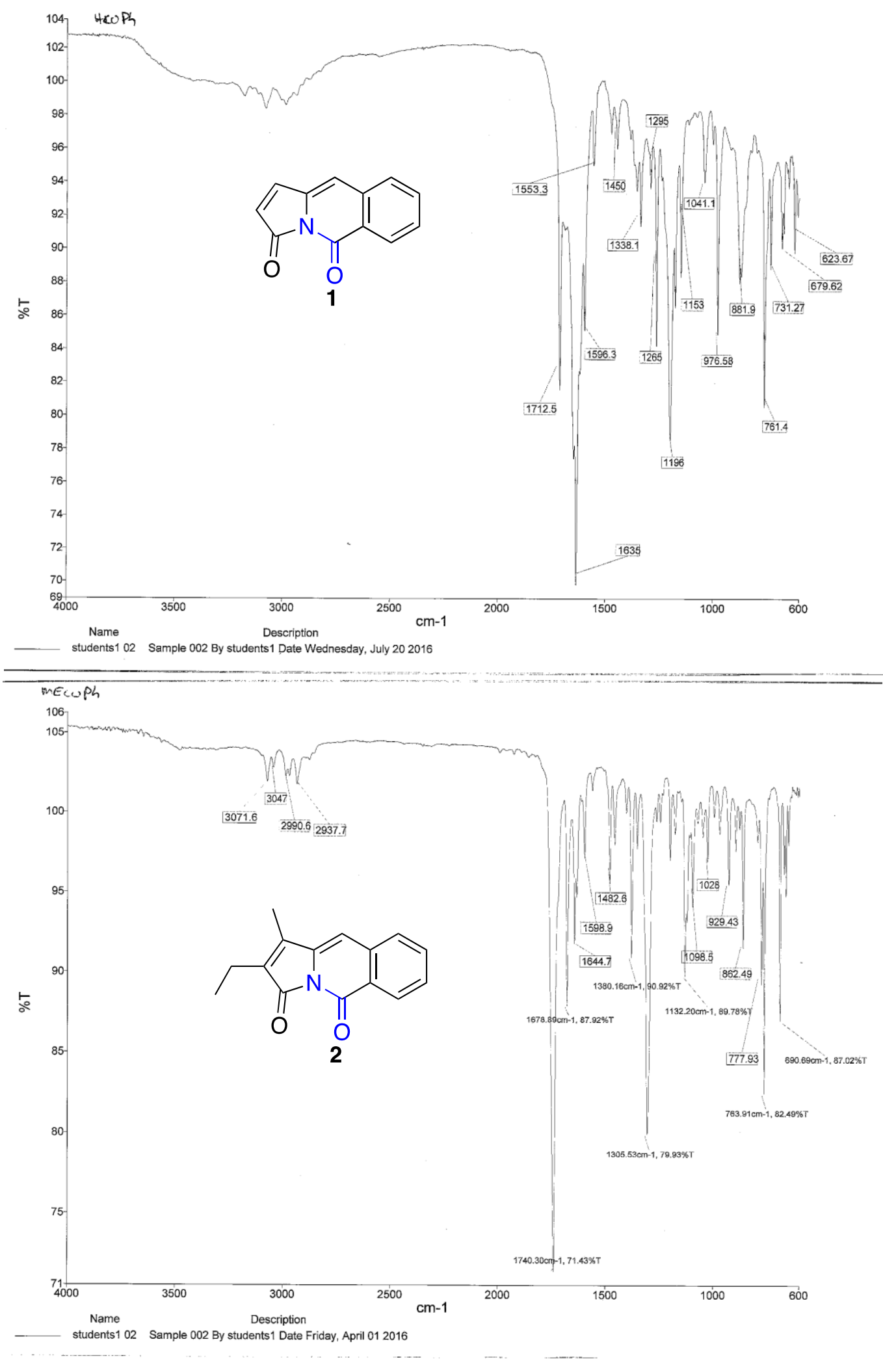


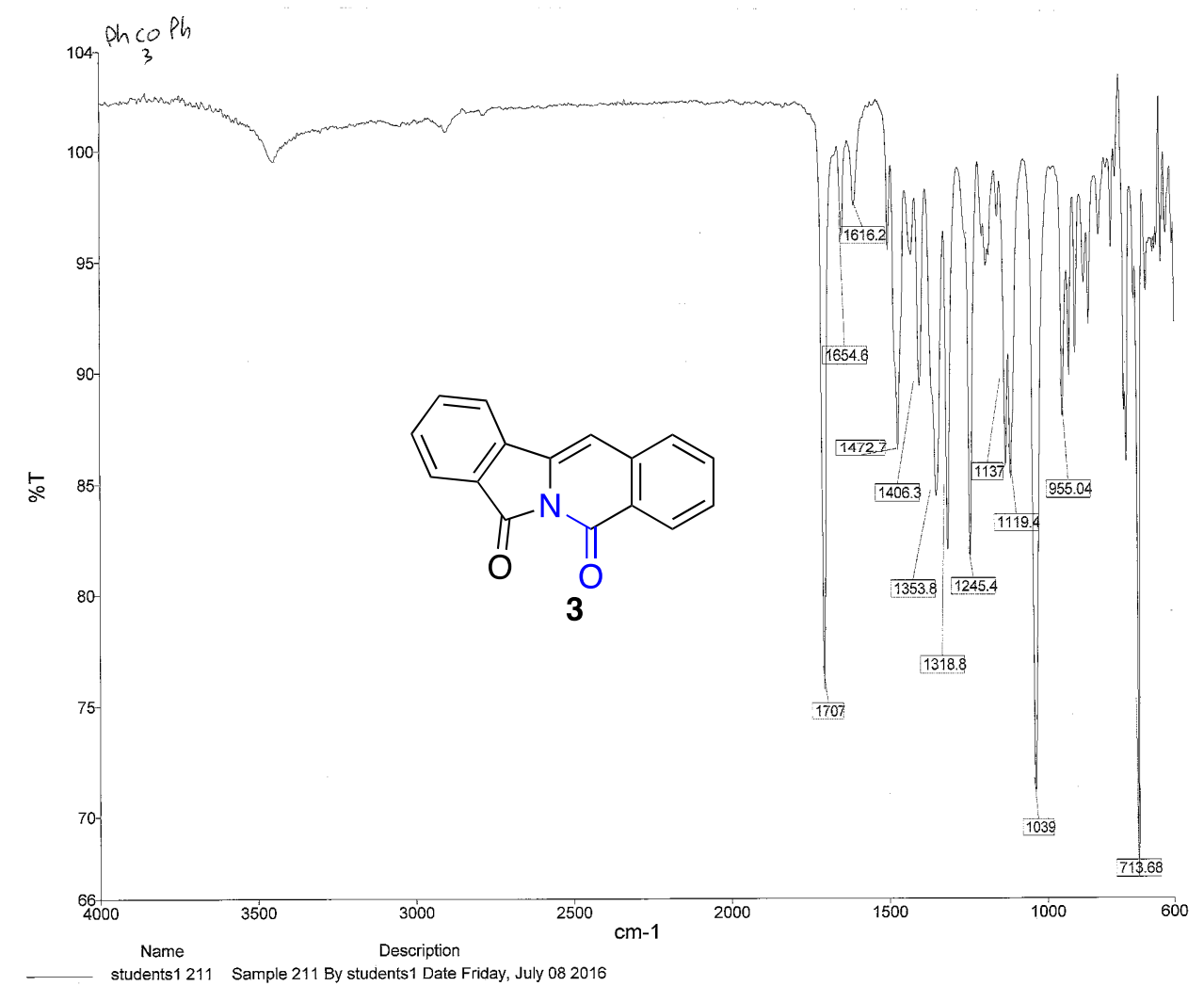

___ students1 211 Sample 211 By students1 Date Friday, July 082016

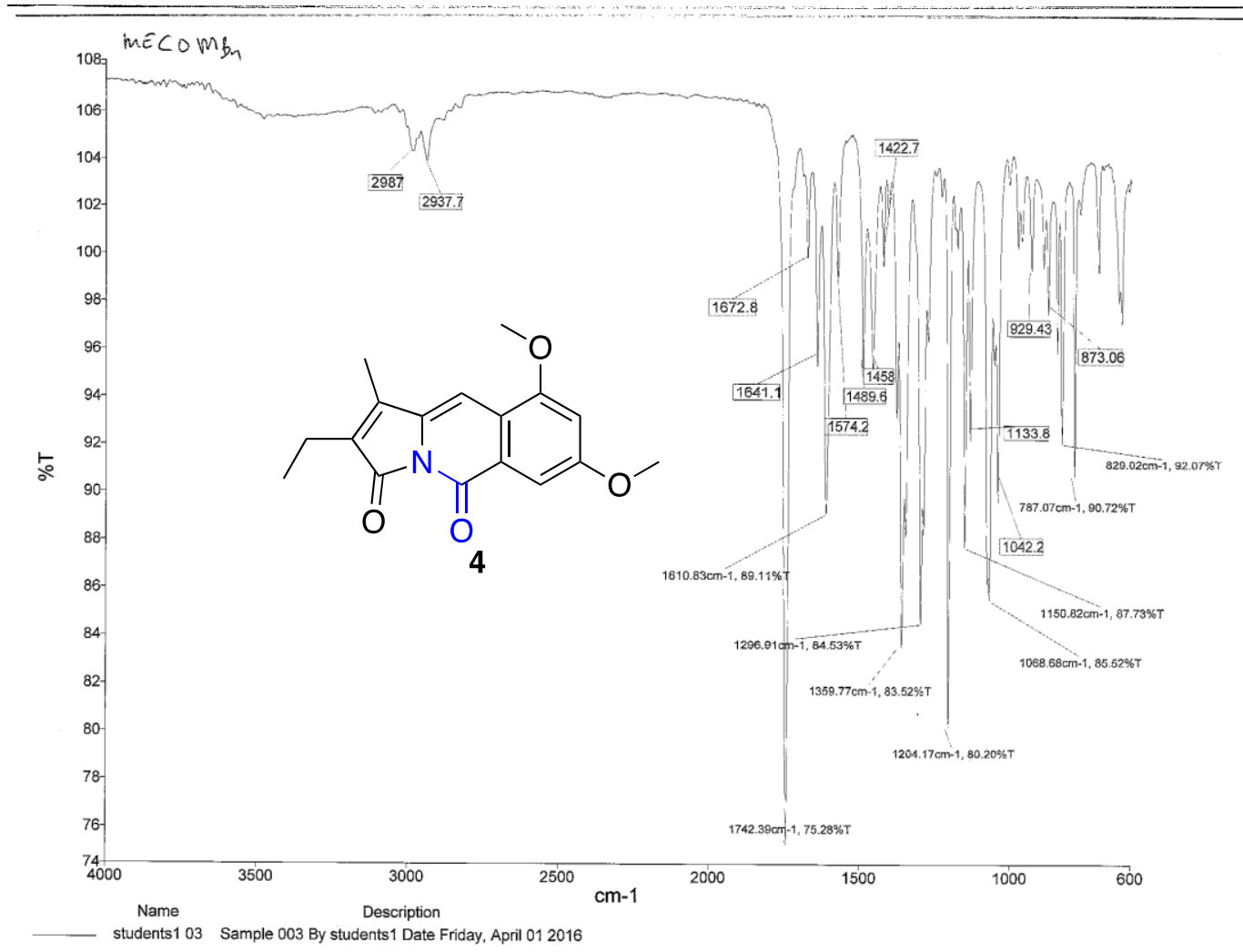



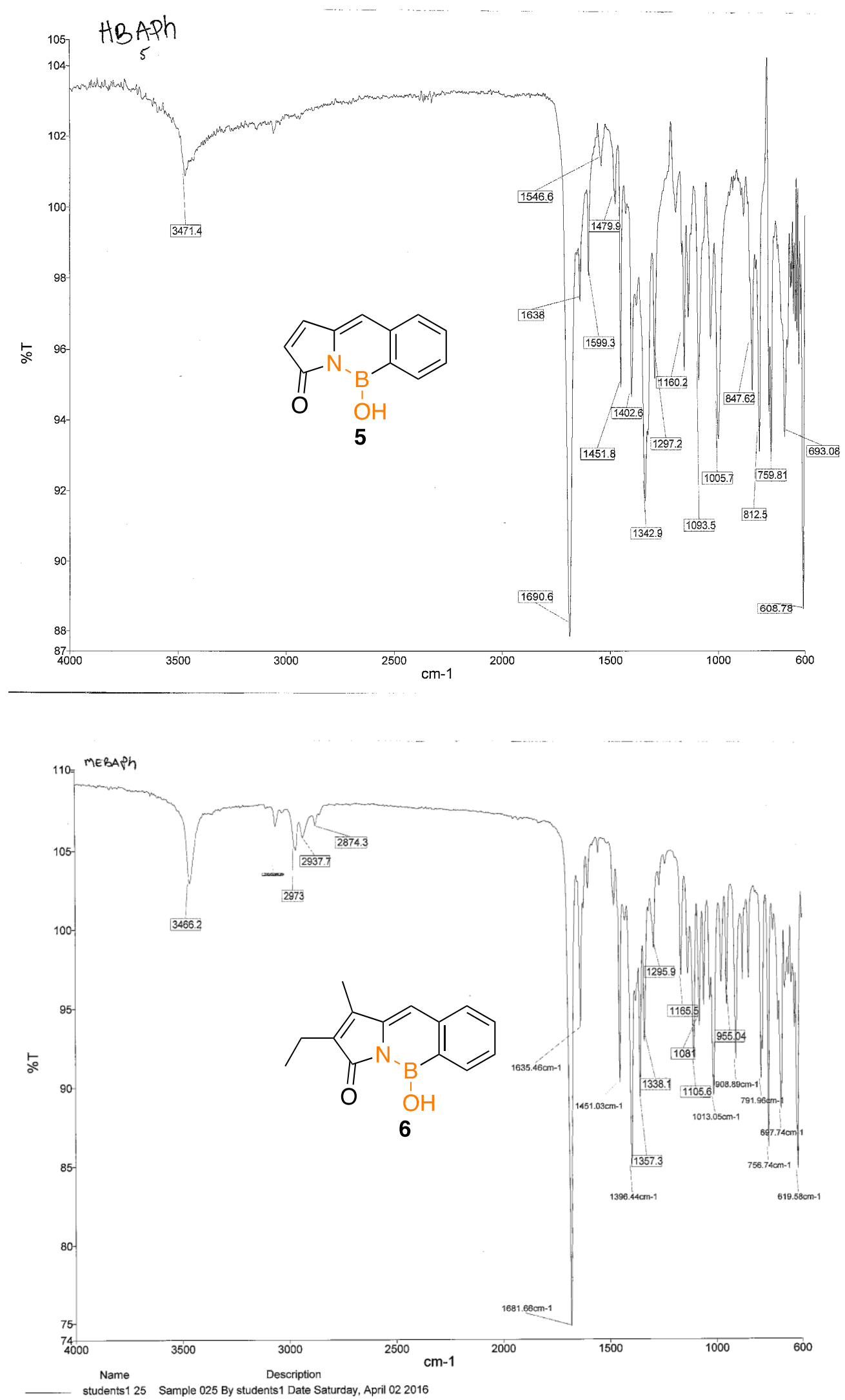

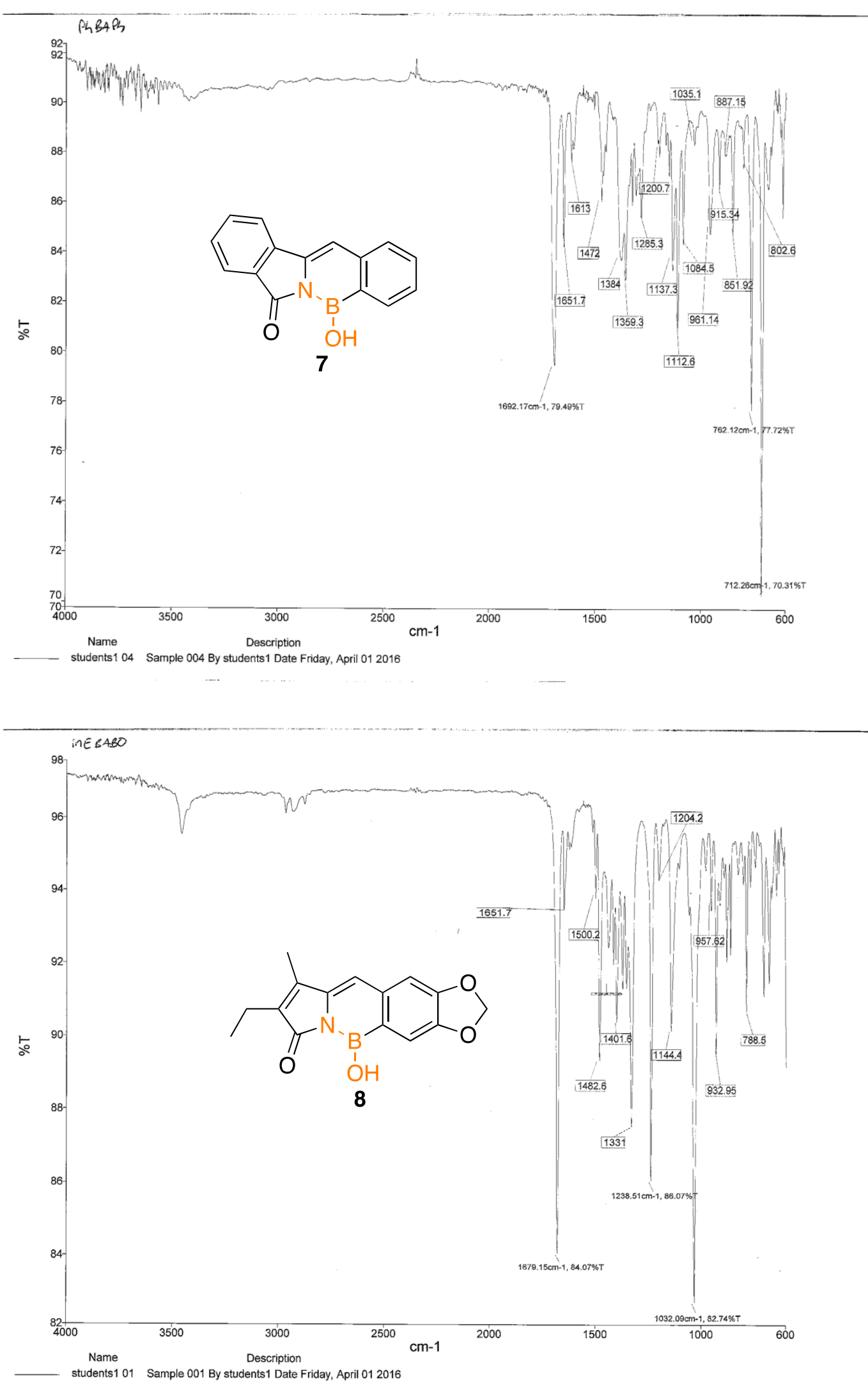

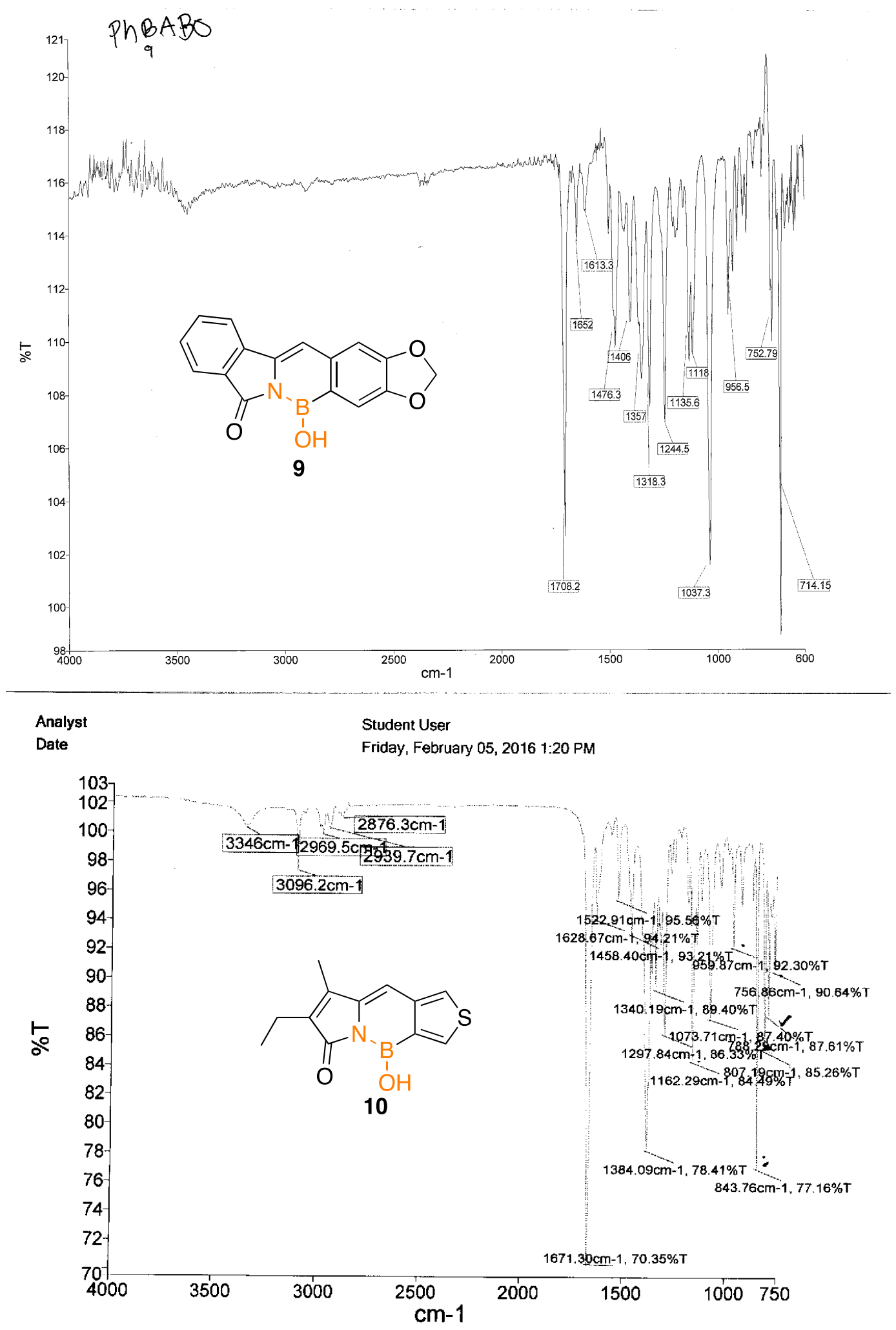
Normalized UV-visible spectra of compounds 1-10 in chloroform

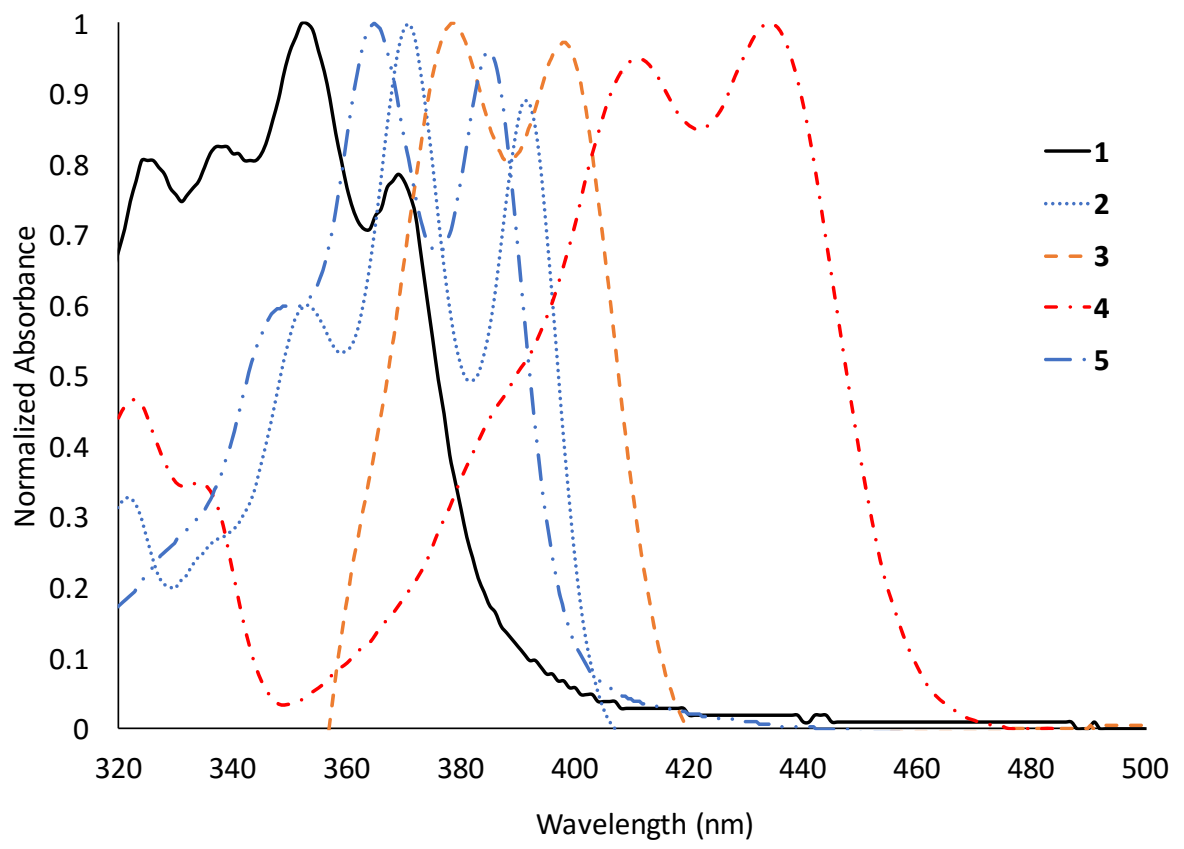

Normalized UV-visible spectra of compounds 1-5 in chloroform

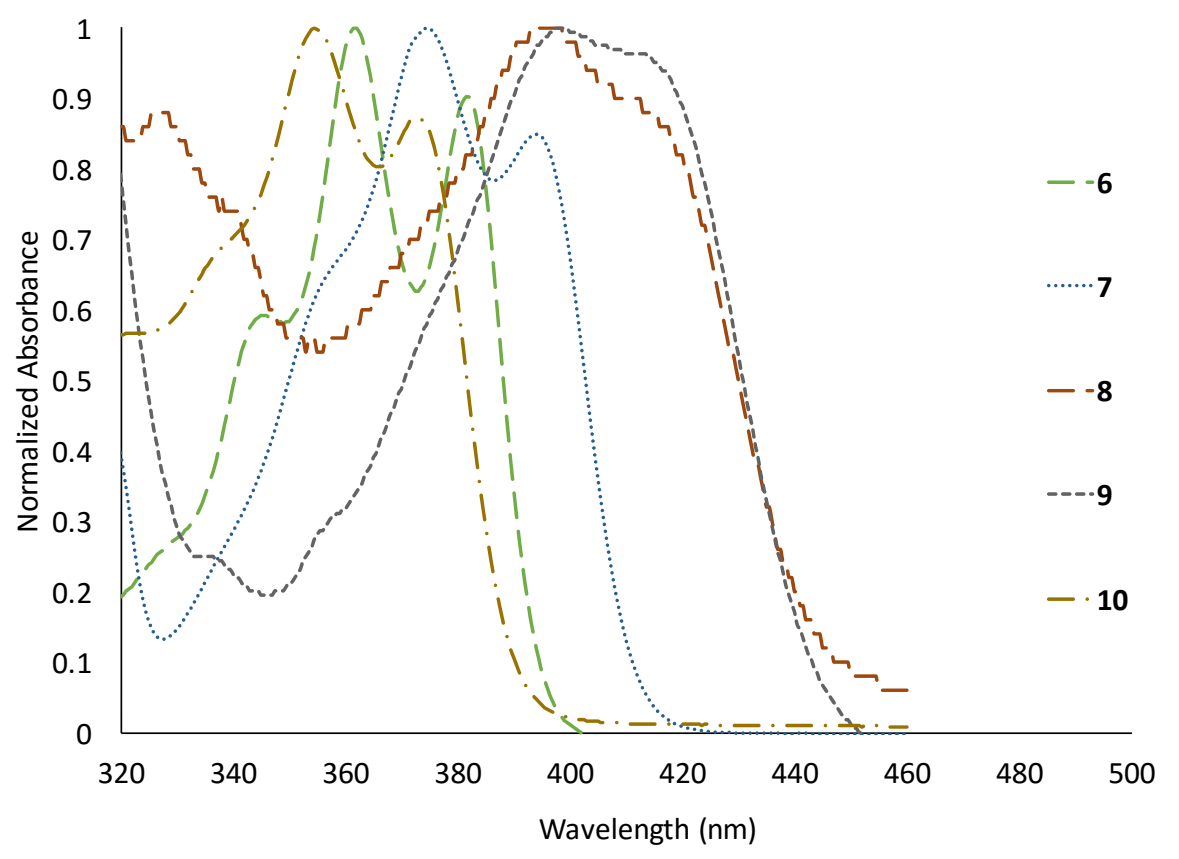

Normalized UV-visible spectra of compounds 6-10 in chloroform 
Normalized UV-visible spectra of compounds 1-10 in methanol

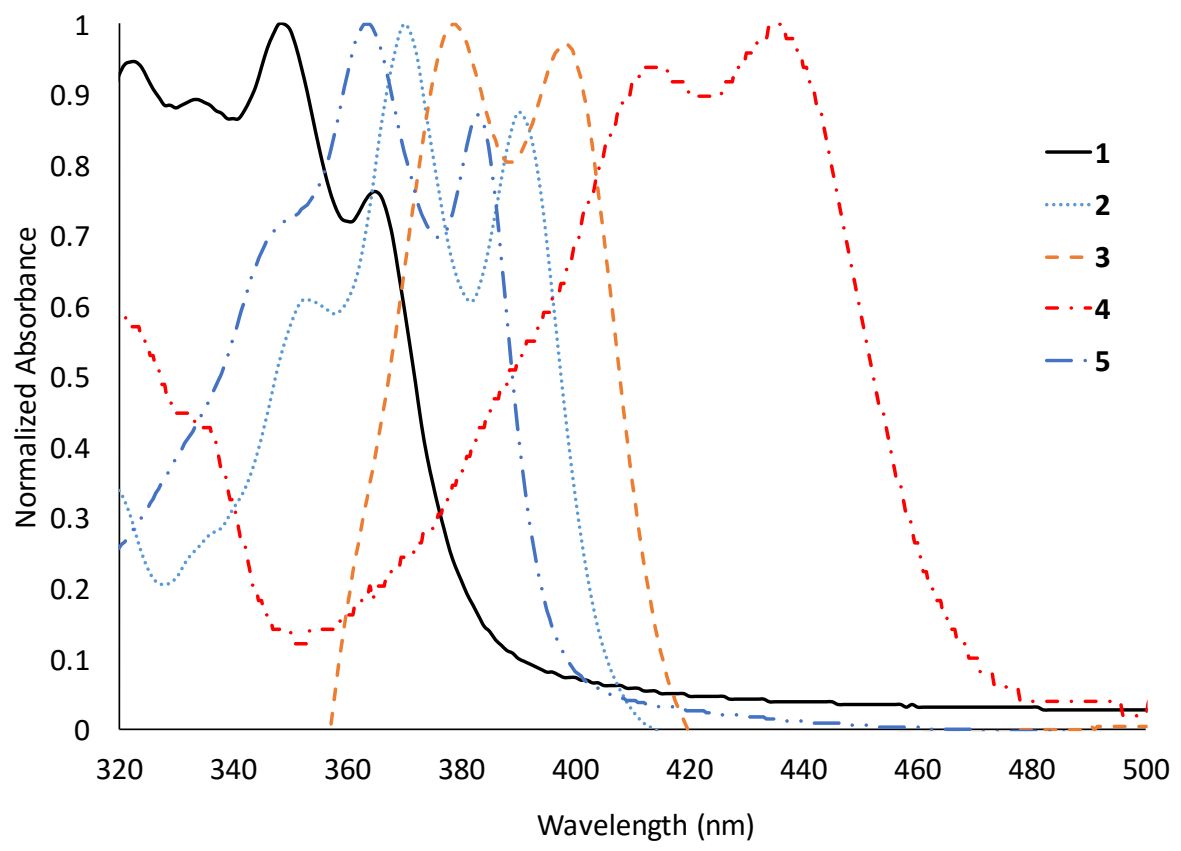

Normalized UV-visible spectra of compounds 1-5 in methanol

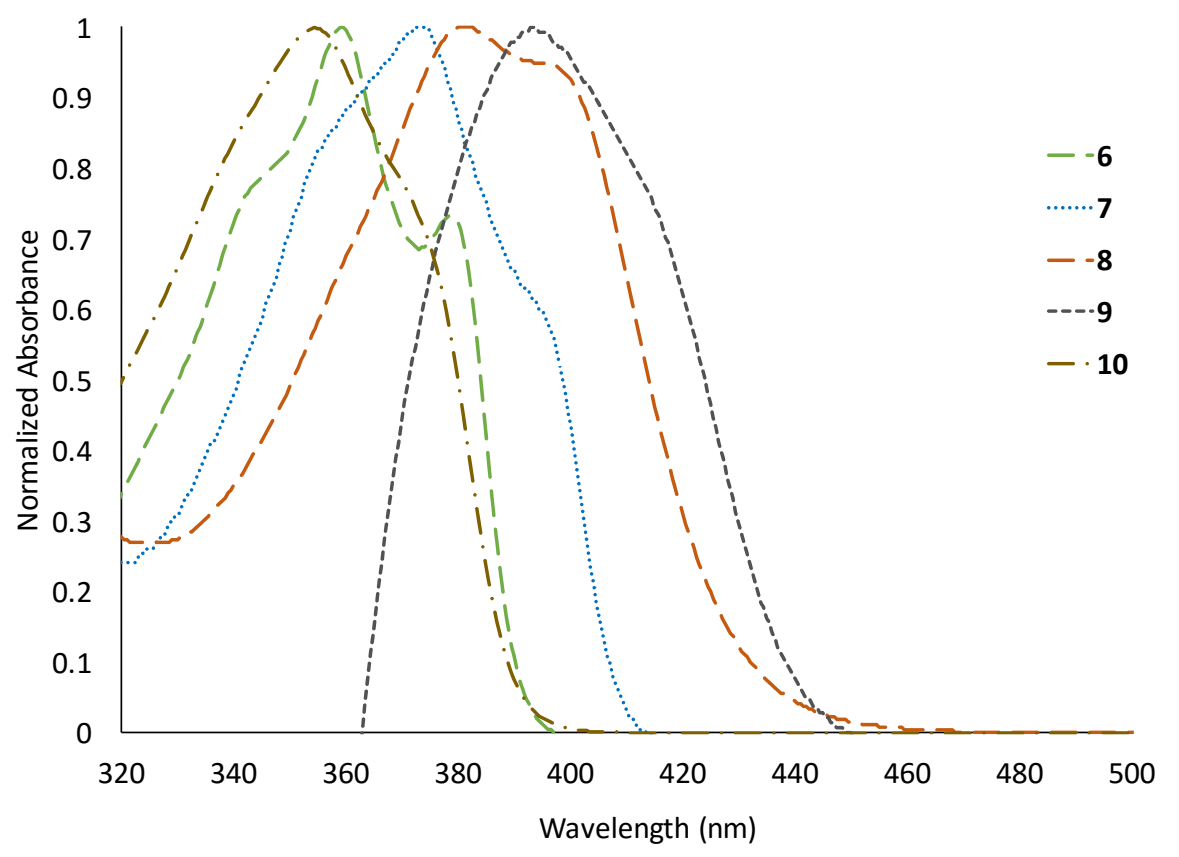

Normalized UV-visible spectra of compounds 6-10 in methanol 


\section{Normalized UV-visible spectra of compounds 1-10 in DMSO}

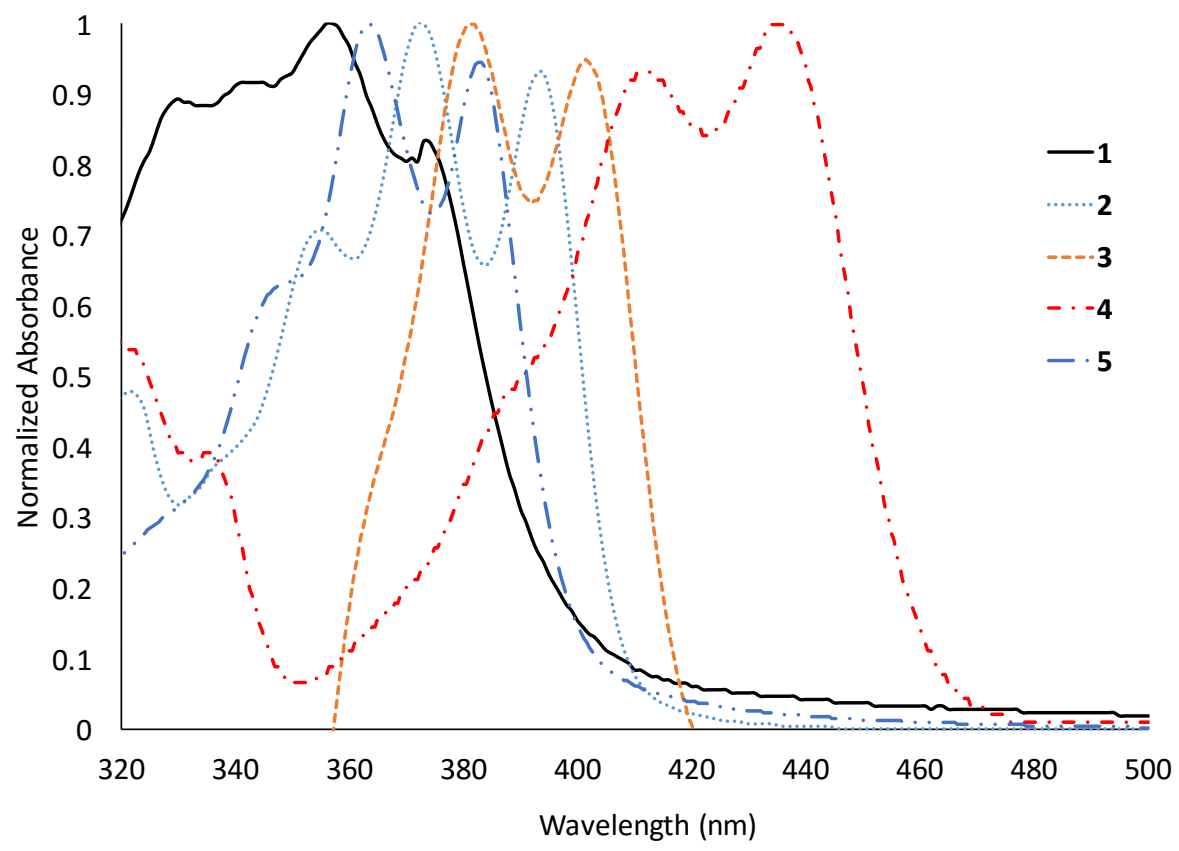

Normalized UV-visible spectra of compounds 1-5 in DMSO

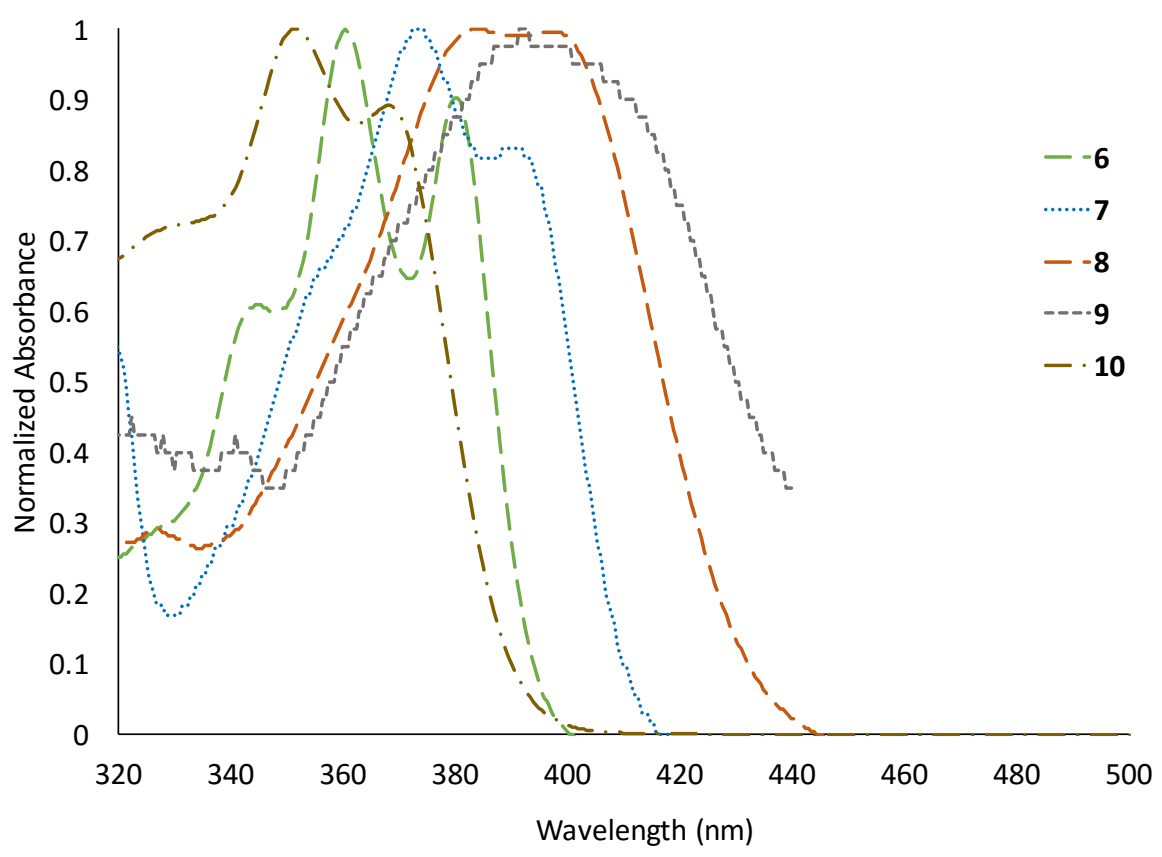

Normalized UV-visible spectra of compounds 6-10 in DMSO 
Normalized UV-visible spectra of compounds 1-10 in acetonitrile

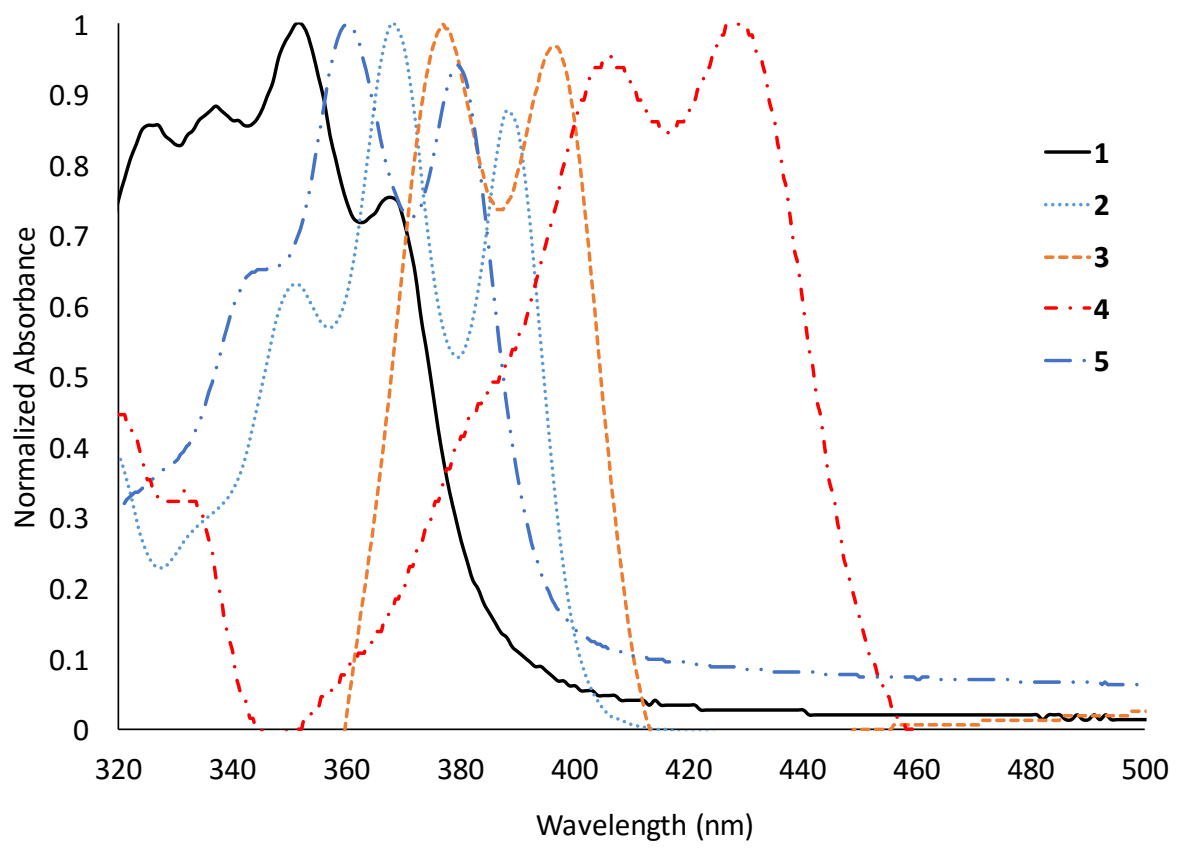

Normalized UV-visible spectra of compounds 1-5 in acetonitrile

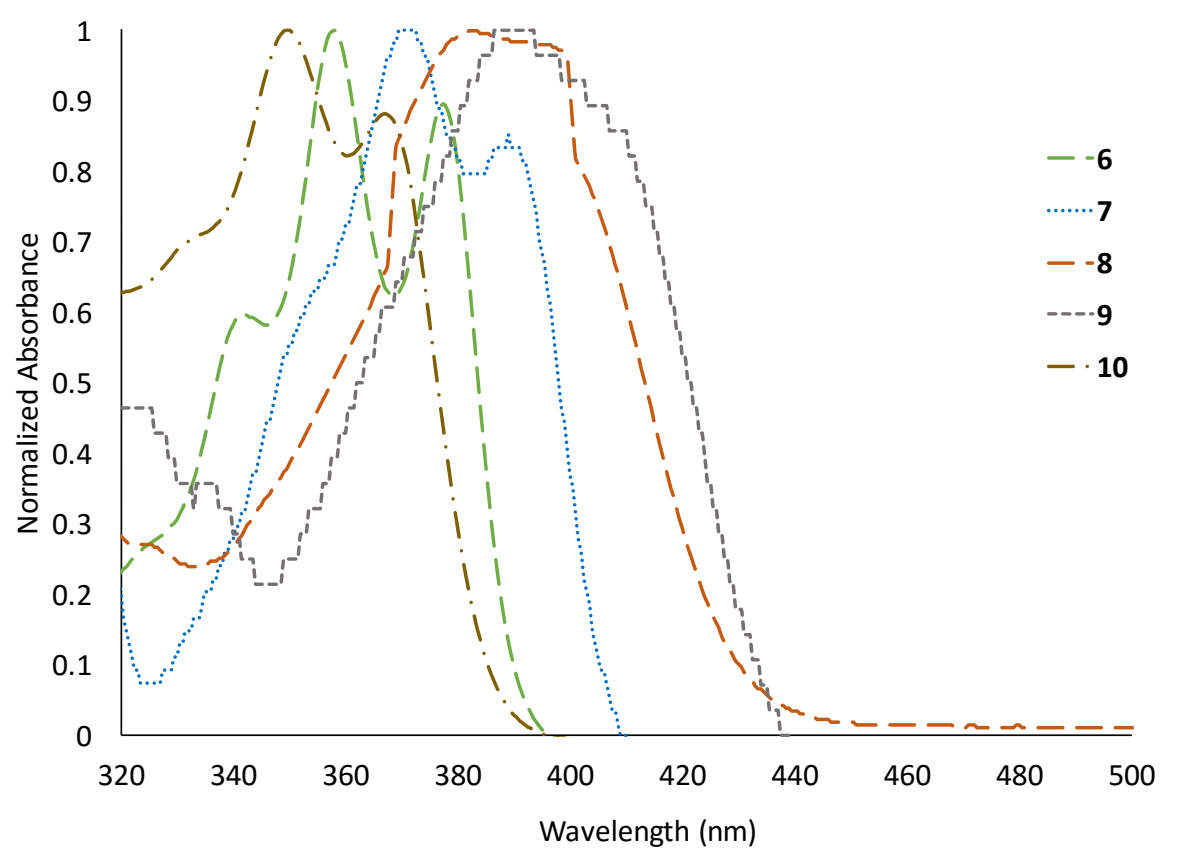

Normalized UV-visible spectra of compounds 6-10 in acetonitrile 
Normalized UV-visible spectra of compounds 6 and 10 in hexanes

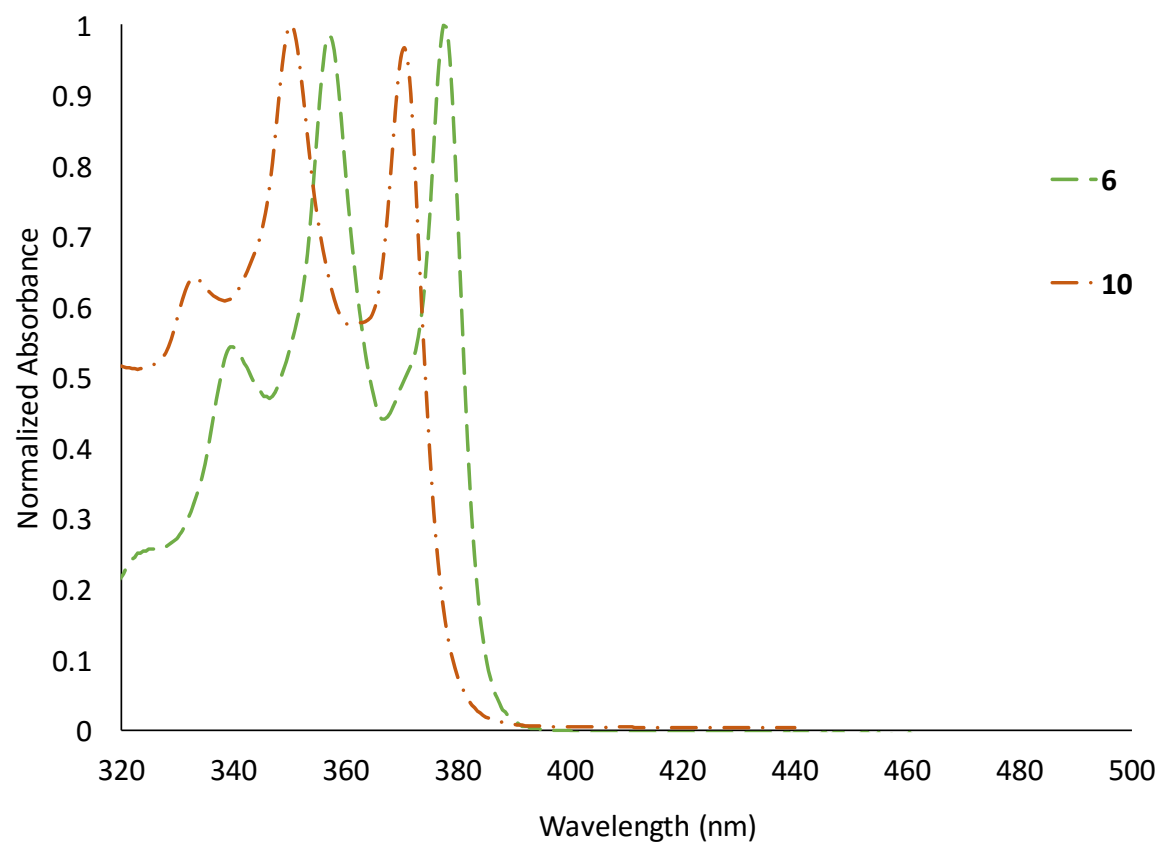

Normalized UV-visible spectra of compounds $\mathbf{6}$ and $\mathbf{1 0}$ in hexanes 


\section{Normalized emission spectra of compounds 5-10 in chloroform.}

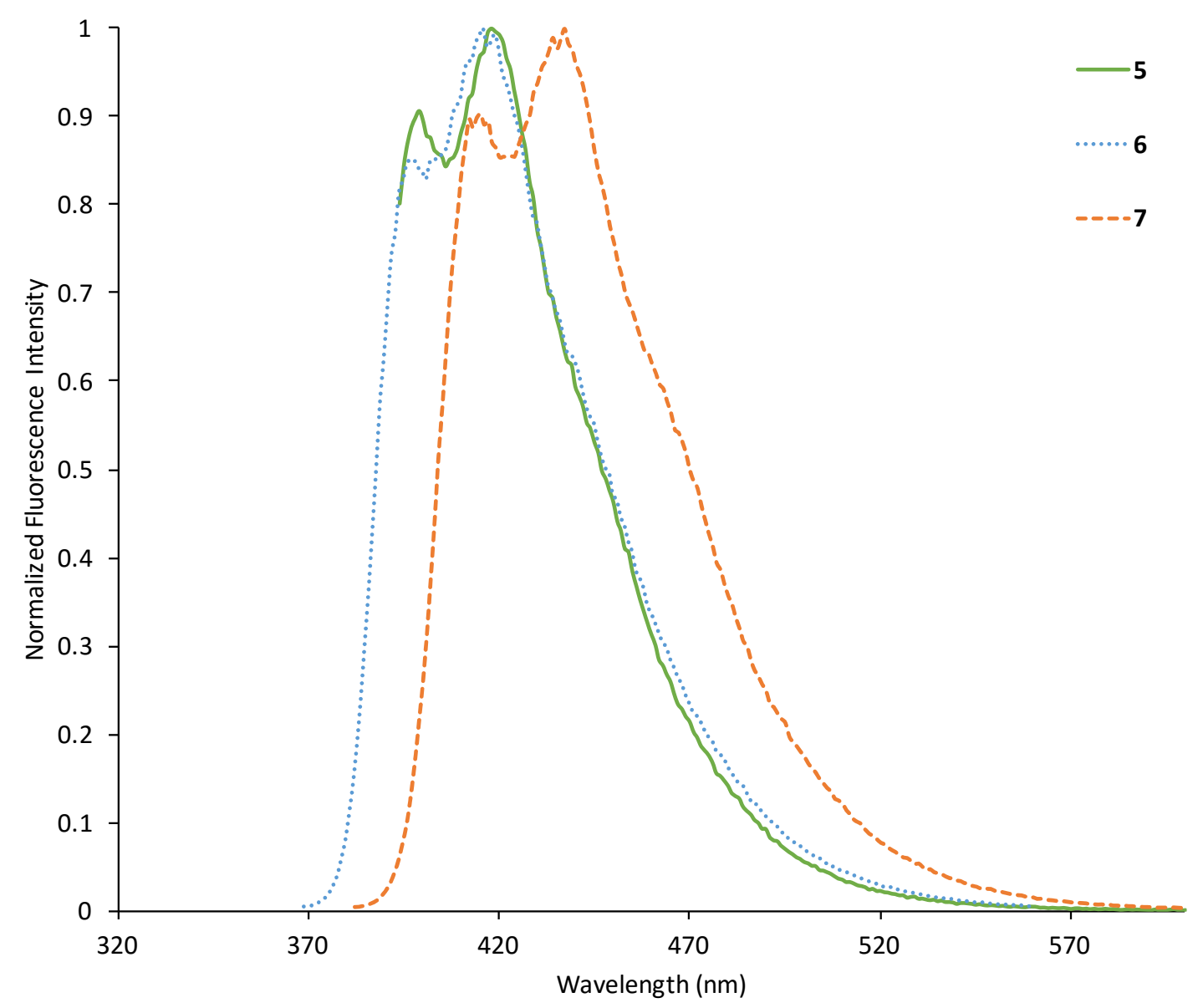

Normalized emission spectra of compounds 5, $\mathbf{6}$ and $\mathbf{7}$ in chloroform 


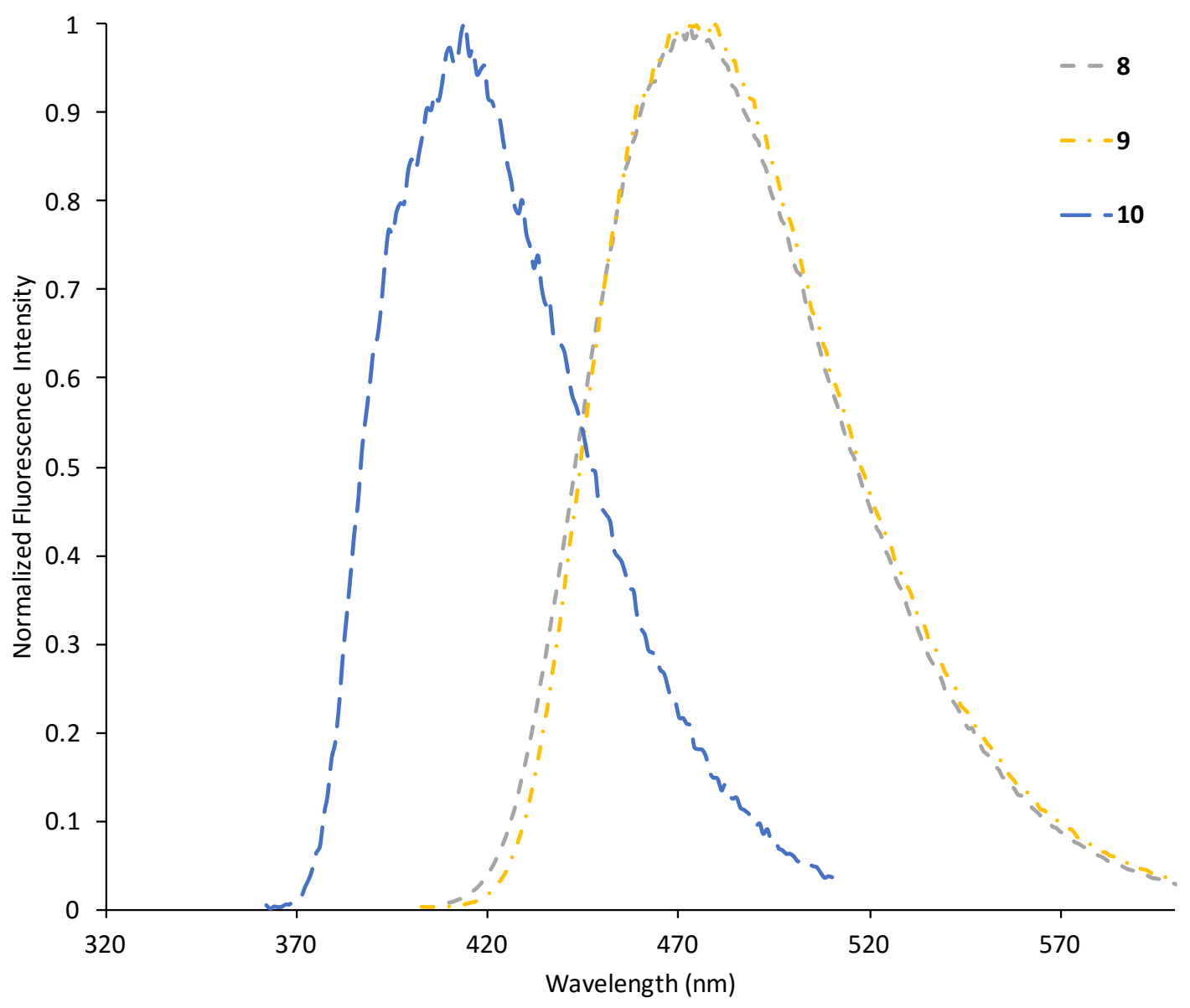

Normalized emission spectra of compounds $\mathbf{8 , 9}$ and $\mathbf{1 0}$ in chloroform 


\section{Normalized emission spectra of compounds 1-10 in the solid state.}

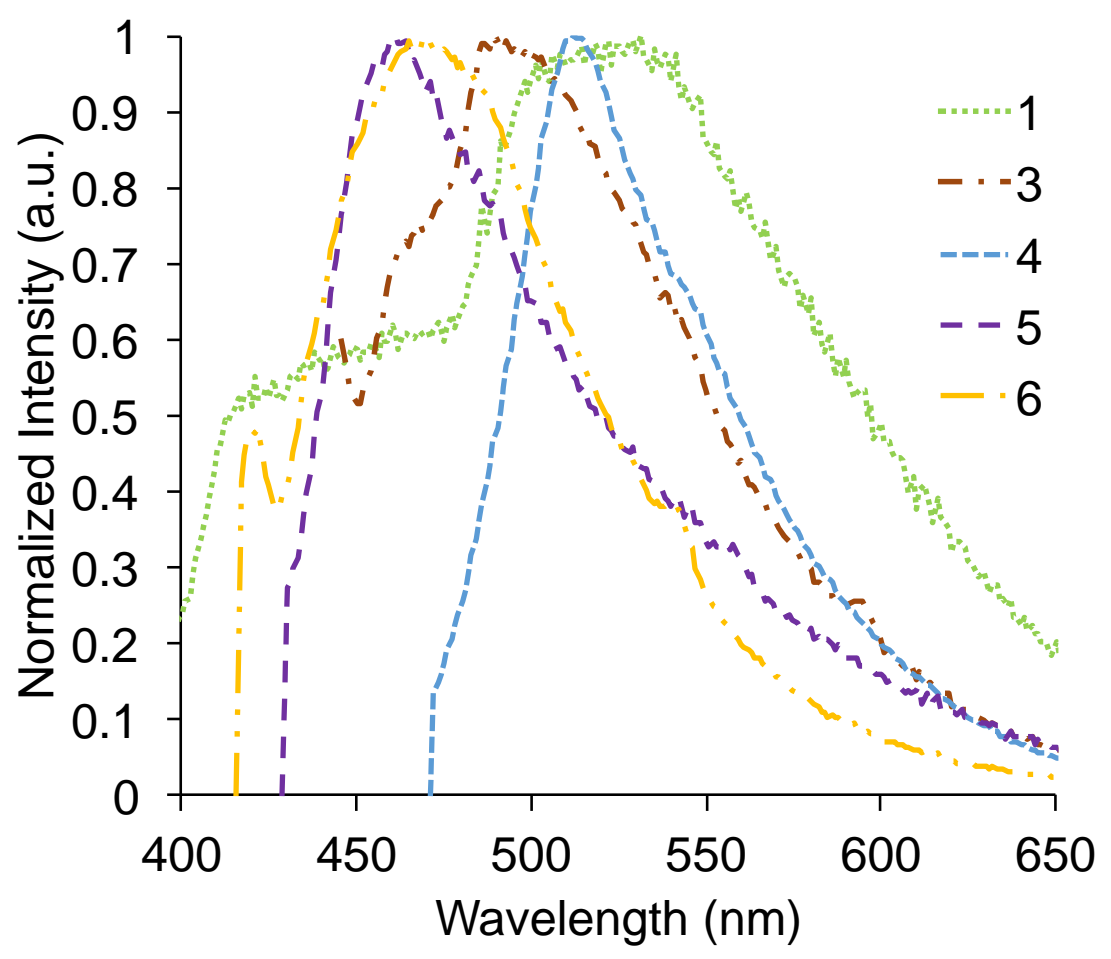

Normalized emission spectra of compounds $\mathbf{1 - 6}$ in the solid state

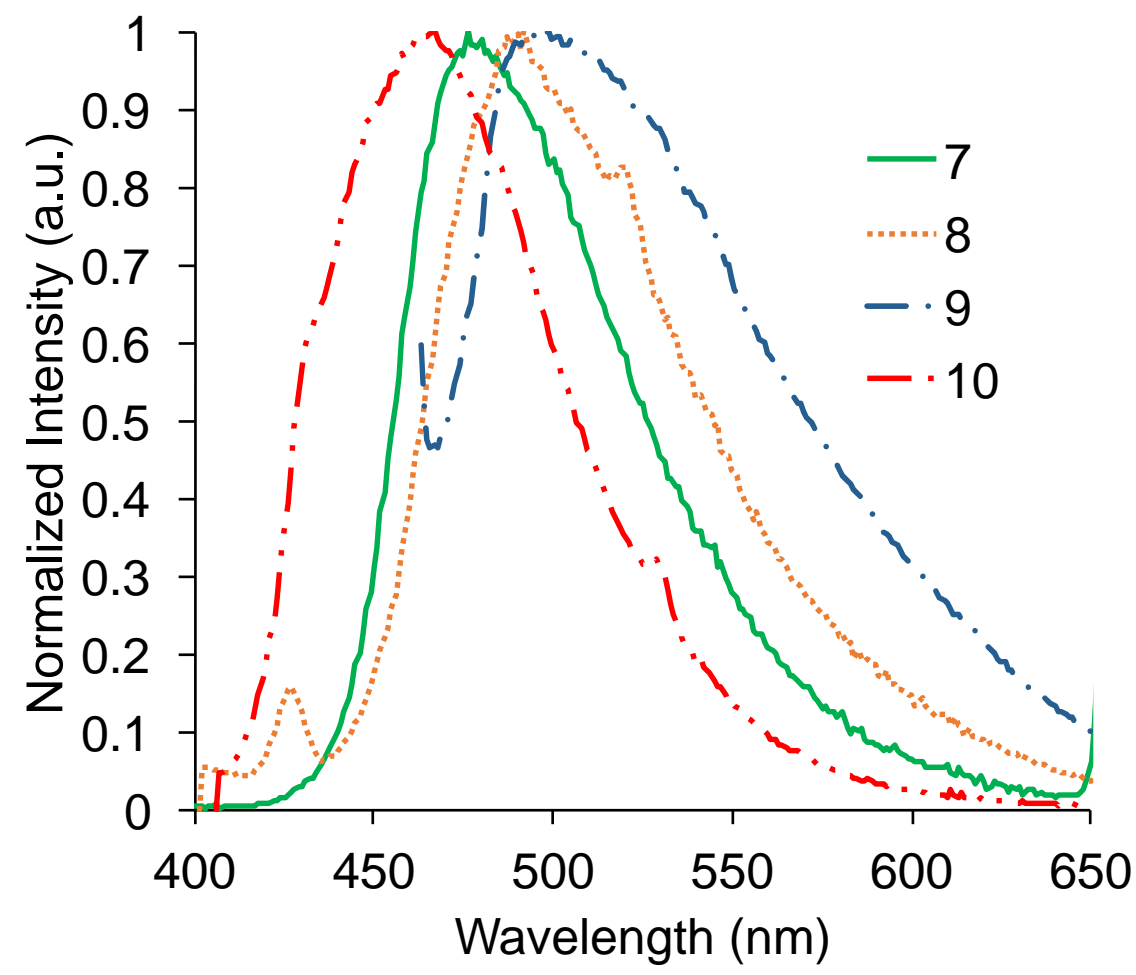

Normalized emission spectra of compounds $\mathbf{7 - 1 0}$ in the solid state 
Normalized UV-visible and emission spectra of compounds 5-10 in chloroform.

A)

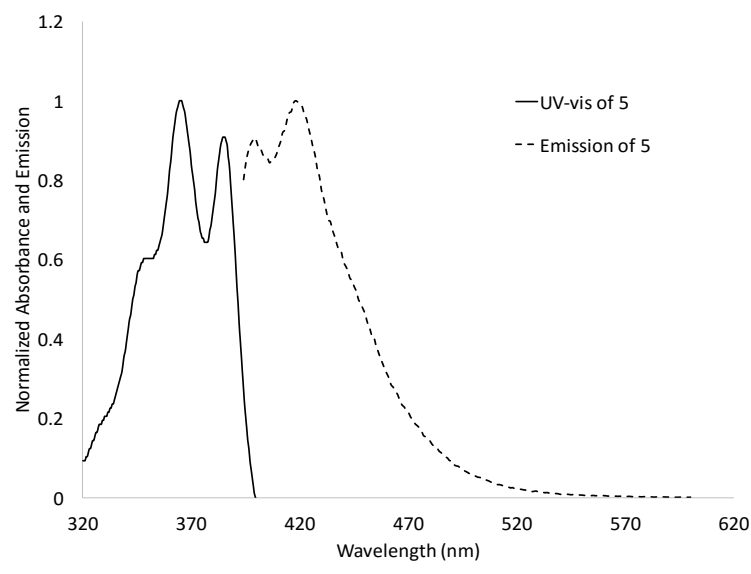

C)

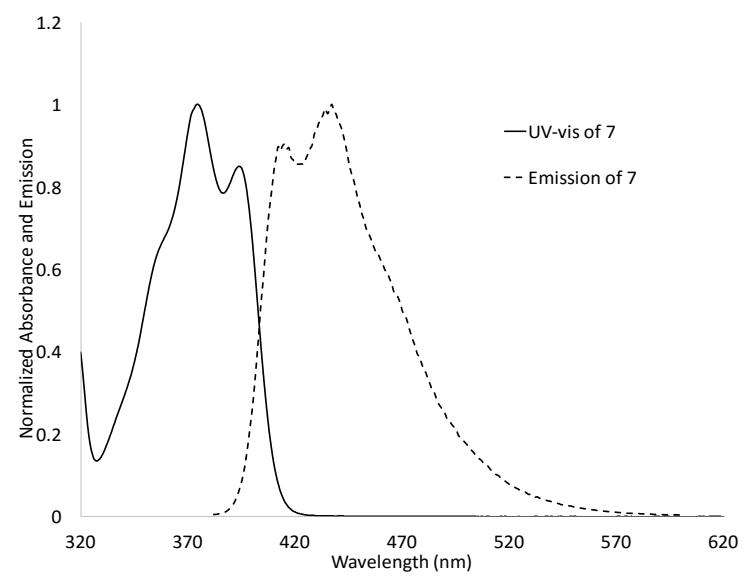

E)

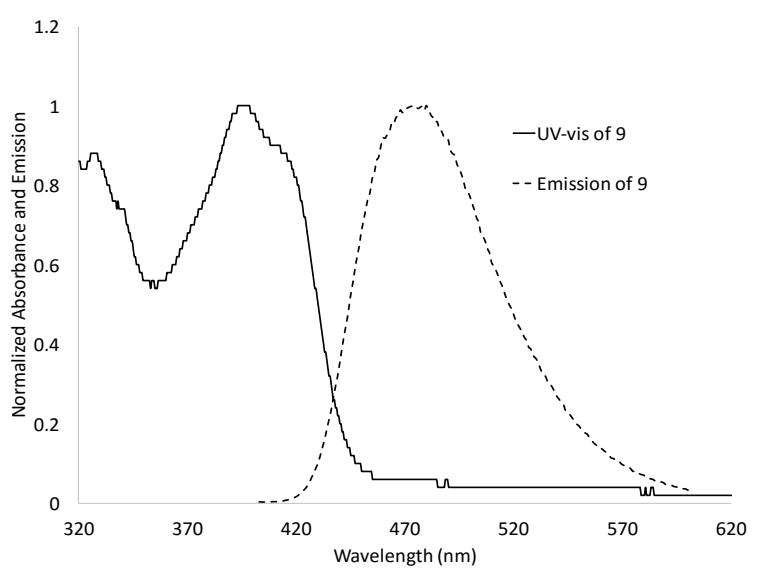

B)

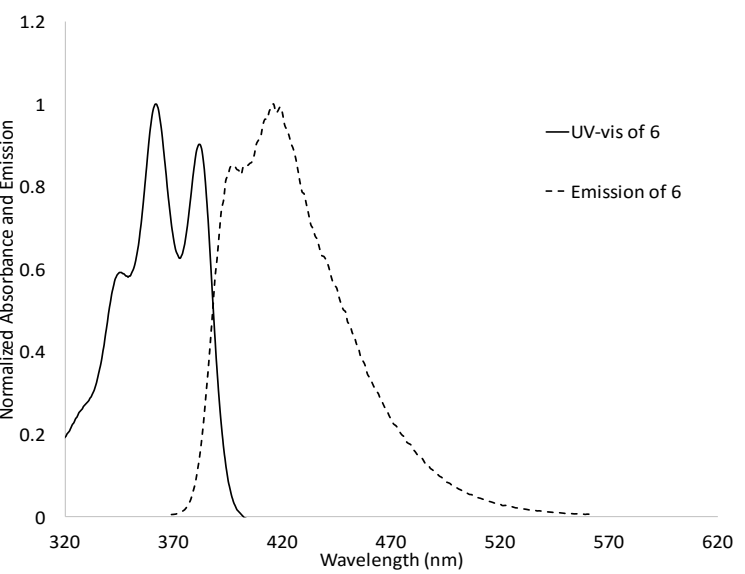

D)

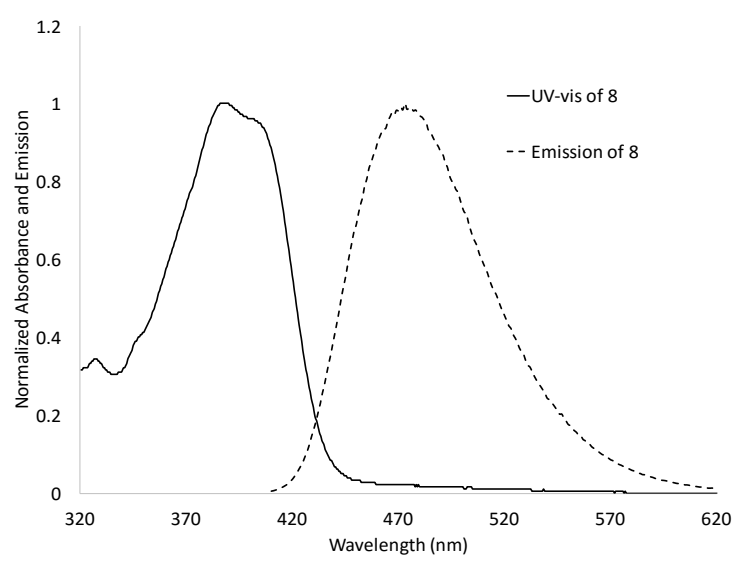

F)

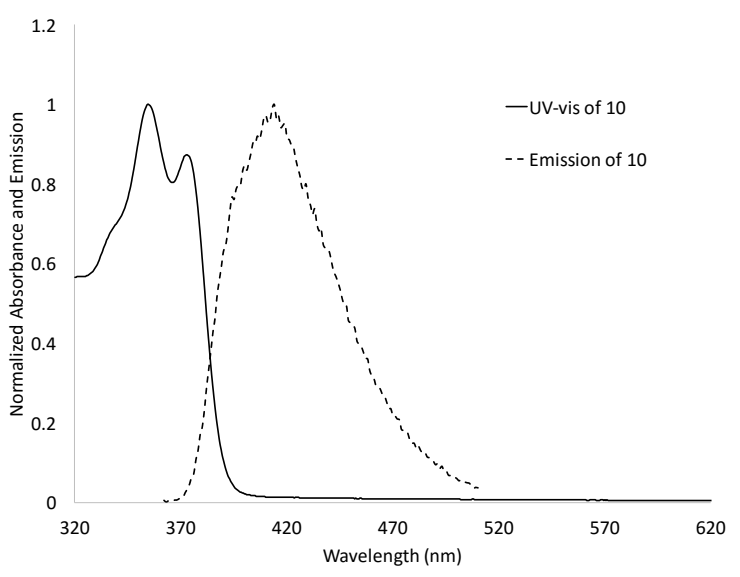


Normalized excitation spectra of compounds 3-10 in the solid state.

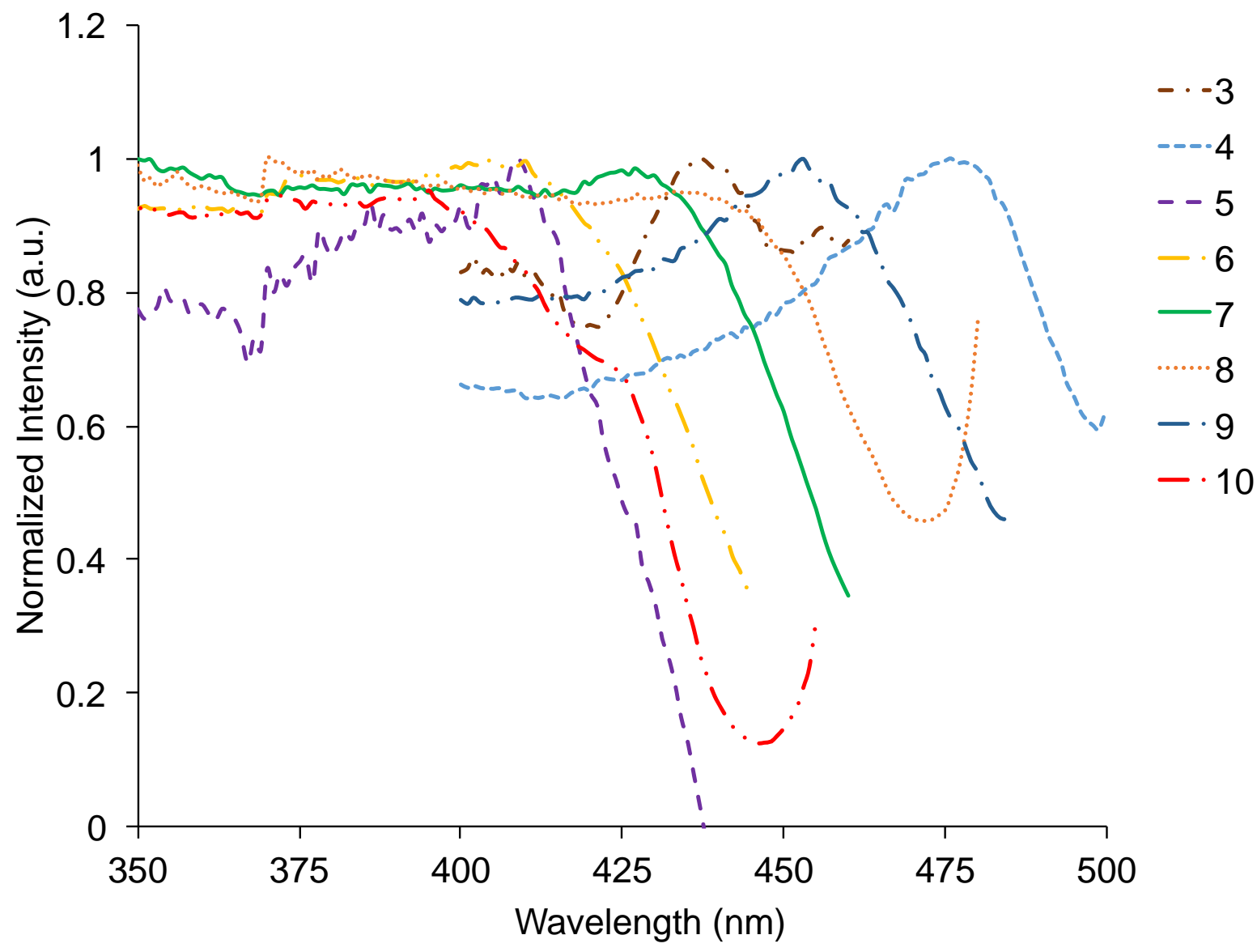

Normalized excitation spectra of compounds 3-10 in the solid state 
Table S1. B3LYP/6-311 ${ }^{++} \mathrm{G}^{* *}$ molecular orbital calculations of 1-12

\begin{tabular}{|ccccc|}
\hline Compound & HOMO $(\mathrm{eV})$ & LUMO $(\mathrm{eV})$ & $\Delta(\mathrm{eV})$ & nm (est abs) \\
\hline $\mathbf{1}$ & -6.56 & -2.97 & -3.59 & 345 \\
\hline $\mathbf{2}$ & -6.35 & -2.67 & -3.68 & 337 \\
\hline $\mathbf{3}$ & -6.36 & -2.68 & -3.68 & 337 \\
\hline $\mathbf{4}$ & -5.78 & -2.46 & -3.32 & 373 \\
\hline $\mathbf{5}$ & -6.35 & -2.81 & -3.54 & 350 \\
\hline $\mathbf{6}$ & -6.15 & -2.52 & -3.63 & 342 \\
\hline $\mathbf{7}$ & -6.15 & -2.54 & -3.61 & 343 \\
\hline $\mathbf{8}$ & -5.81 & -2.46 & -3.35 & 370 \\
\hline $\mathbf{9}$ & -5.79 & -2.47 & -3.32 & 373 \\
\hline $\mathbf{1 0}$ & -6.04 & -2.38 & -3.66 & 339 \\
\hline $\mathbf{2 0}$ & -6.60 & -3.04 & -3.56 & 348 \\
\hline $\mathbf{2 1}$ & -5.74 & -0.8 & -4.94 & 251 \\
\hline
\end{tabular}

Table S2. Stokes shift (nm) of compounds 3-10 in chloroform, methanol, DMSO, and acetonitrile

\begin{tabular}{|ccccc|}
\hline Compounds & $\mathbf{C H C l}_{\mathbf{3}}$ & $\mathrm{CH}_{\mathbf{3}} \mathrm{OH}$ & DMSO & $\mathbf{C H}_{3} \mathbf{C N}$ \\
\hline $\mathbf{3}$ & 66 & 66 & 73 & 66 \\
\hline $\mathbf{4}$ & 35 & 77 & 51 & 43 \\
\hline $\mathbf{5}$ & 55 & 52 & 66 & 52 \\
\hline $\mathbf{6}$ & 56 & 52 & 55 & 53 \\
\hline $\mathbf{7}$ & 62 & 58 & 66 & 61 \\
\hline $\mathbf{8}$ & 85 & 103 & 114 & 105 \\
\hline $\mathbf{9}$ & 77 & 96 & 104 & 98 \\
\hline $\mathbf{1 0}$ & 59 & 63 & 73 & 62 \\
\hline
\end{tabular}

Table S3. Emission of 3-10 in the solid state.

\begin{tabular}{|c|c|}
\hline Compounds & $\begin{array}{c}\text { Solid State } \\
\text { Emission } \\
\text { (nm) }\end{array}$ \\
\hline $\mathbf{1}$ & 520 \\
\hline $\mathbf{3}$ & 489 \\
\hline $\mathbf{4}$ & 510 \\
\hline $\mathbf{5}$ & 459 \\
\hline $\mathbf{6}$ & 468 \\
\hline $\mathbf{7}$ & 474 \\
\hline $\mathbf{8}$ & 490 \\
\hline $\mathbf{9}$ & 502 \\
\hline $\mathbf{1 0}$ & 465 \\
\hline
\end{tabular}


A)

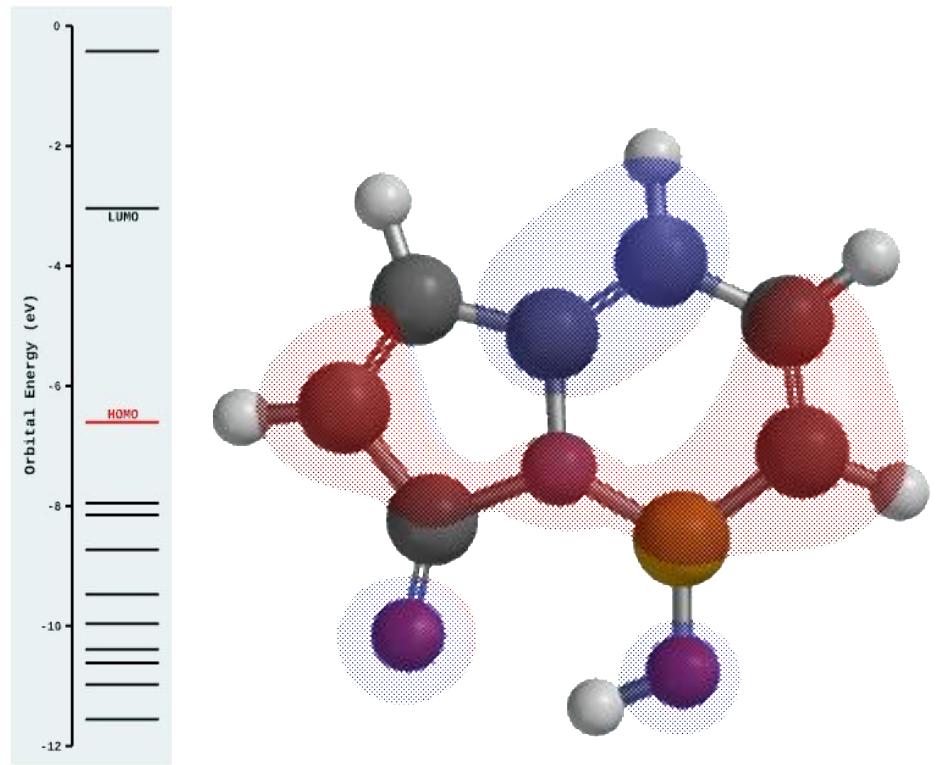

B)

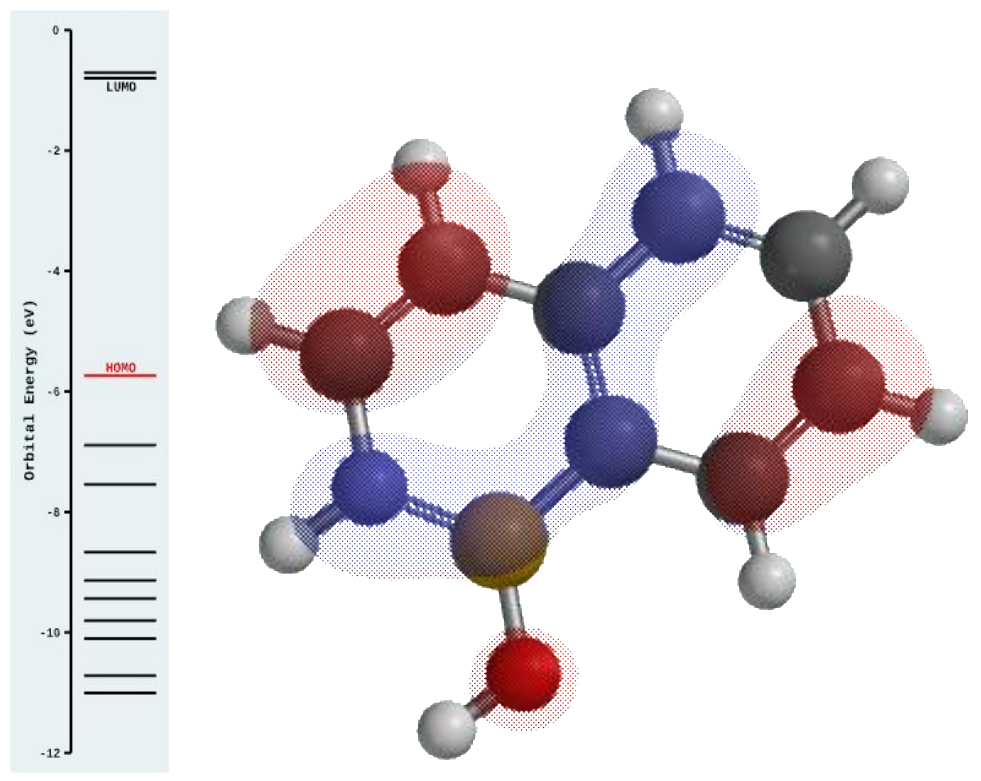

B3LYP/6-311 ${ }^{++} \mathrm{G}^{* *}$ molecular orbital calculations of A) Left hemisphere of 20 responsible for the absorbance at $365 \mathrm{~nm} \mathrm{~B}$ ) Right hemisphere of 21. 


\section{A) Modeling Results for 20}

MacSPARTAN '14 Quantum Mechanics Program: (x86/Darwin) build 14.119

\section{$\mathrm{C}_{7} \mathrm{H}_{6} \mathrm{BNO} 2$}

Method: RB3LYP

Basis set: $6-311++\mathrm{G}^{* *}$

Number of shells: 96

Number of basis functions: 284

Multiplicity: 1

\section{Energy: -500.646063 au}

Coordinates (Angstroms)

\begin{tabular}{llll} 
ATOM & \multicolumn{1}{c}{$\mathrm{X}$} & \multicolumn{1}{c}{$\mathrm{Y}$} & \multicolumn{1}{c}{$\mathrm{Z}$} \\
$1 \mathrm{C}$ & -1.382059 & -1.656536 & 0.000000 \\
2 C & -2.260159 & 0.637471 & 0.000000 \\
3 N & 0.204049 & 0.136638 & 0.000000 \\
4 B & -0.818299 & 1.179754 & 0.000000 \\
5 C & -0.094944 & -1.220832 & 0.000000 \\
6 C & -2.469720 & -0.708172 & 0.000000 \\
7 C & 1.170904 & -1.946411 & 0.000000 \\
8 C & 2.186320 & -1.059616 & 0.000000 \\
9 C & 1.609753 & 0.303396 & 0.000000 \\
$10 \mathrm{O}$ & 2.164430 & 1.387339 & 0.000000 \\
$11 \mathrm{O}$ & -0.476265 & 2.494371 & 0.000000 \\
$12 \mathrm{H}$ & -1.585278 & -2.720387 & 0.000000 \\
$13 \mathrm{H}$ & -3.119400 & 1.301031 & 0.000000 \\
$14 \mathrm{H}$ & -3.478193 & -1.112810 & 0.000000 \\
$15 \mathrm{H}$ & 1.240760 & -3.025096 & 0.000000 \\
$16 \mathrm{H}$ & 3.248770 & -1.246876 & 0.000000 \\
$17 \mathrm{H}$ & 0.486513 & 2.613628 & 0.000000
\end{tabular}

Point Group: cs Number of degrees of freedom: 31 


\section{B) Modeling Results for 21}

MacSPARTAN '14 Quantum Mechanics Program: (x86/Darwin) build 14.119

\section{$\mathrm{C}_{8} \mathrm{H}_{8} \mathrm{BNO}$}

Method: RB3LYP

Basis set: $6-311++\mathrm{G}^{* *}$

Number of shells: 106

Number of basis functions: 298

Multiplicity: 1

Energy: -464.736387 au

Coordinates (Angstroms)

\begin{tabular}{llll} 
ATOM & \multicolumn{1}{c}{$\mathrm{X}$} & \multicolumn{1}{c}{$\mathrm{Y}$} & \multicolumn{1}{c}{$\mathrm{Z}$} \\
1 C & 1.822124 & -1.241143 & 0.000549 \\
2 C & 2.431008 & 1.099782 & -0.000209 \\
3 C & 0.079613 & 0.471240 & -0.001468 \\
4 C & 1.089765 & 1.450350 & -0.001299 \\
5 C & 0.453175 & -0.900819 & -0.000472 \\
6 C & 2.793848 & -0.255446 & 0.000759 \\
7 C & -0.570807 & -1.922299 & -0.000288 \\
8 C & -1.885692 & -1.608402 & -0.000243 \\
9 N & -2.326119 & -0.293975 & -0.000155 \\
$10 \mathrm{~B}$ & -1.419813 & 0.821003 & -0.001015 \\
$11 \mathrm{O}$ & -1.854514 & 2.130771 & -0.000607 \\
$12 \mathrm{H}$ & 2.111065 & -2.287585 & 0.001344 \\
$13 \mathrm{H}$ & 3.198373 & 1.866107 & 0.000016 \\
$14 \mathrm{H}$ & 0.802174 & 2.496292 & -0.001998 \\
$15 \mathrm{H}$ & 3.842637 & -0.533349 & 0.001795 \\
$16 \mathrm{H}$ & -0.280205 & -2.965859 & 0.000064 \\
$17 \mathrm{H}$ & -2.654195 & -2.372262 & -0.000130 \\
$18 \mathrm{H}$ & -3.328825 & -0.173422 & -0.000609 \\
$19 \mathrm{H}$ & -2.807210 & 2.252519 & 0.005141
\end{tabular}

Point Group: c1 Number of degrees of freedom: 51 


\section{Modeling Results for 1}
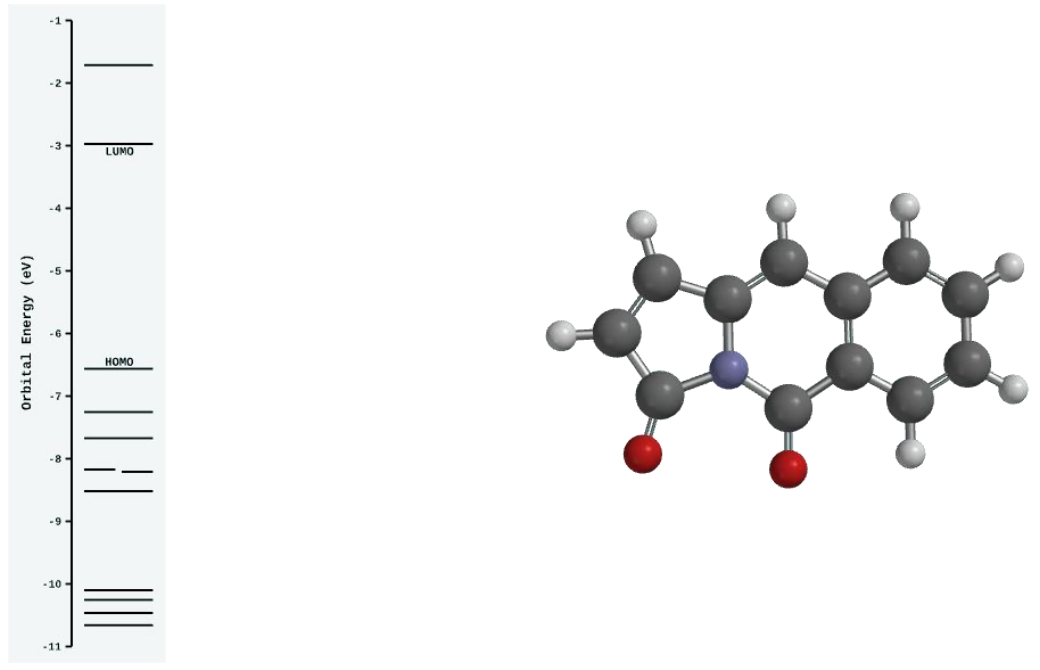

1

MacSPARTAN '14 Quantum Mechanics Program: (x86/Darwin) build 14.119

\section{$\mathrm{C}_{12} \mathrm{H}_{7} \mathrm{NO}_{2}$}

Method: RB3LYP

Basis set: $6-311++\mathrm{G}^{* *}$

Number of shells: 125

Number of basis functions: 379

Multiplicity: 1

Energy: -666.881816 au

Coordinates (Angstroms)

\begin{tabular}{llll} 
ATOM X & \multicolumn{1}{c}{ Y } & \multicolumn{1}{l}{$\mathrm{Z}$} \\
1 C & 2.628709 & -1.416227 & 0.000000 \\
2 C & 3.533941 & 0.824924 & 0.000000 \\
3 C & 1.139042 & 0.503539 & 0.000000 \\
4 C & 2.249867 & 1.352196 & 0.000000 \\
5 C & 1.320600 & -0.899840 & 0.000000 \\
6 C & 3.721215 & -0.562707 & 0.000000 \\
7 C & 0.160476 & -1.755897 & 0.000000 \\
8 C & -1.073693 & -1.206136 & 0.000000 \\
9 N & -1.271186 & 0.179755 & 0.000000 \\
10 C & -0.217234 & 1.120557 & 0.000000 \\
11 C & -2.691188 & 0.459064 & 0.000000 \\
12 C & -3.333115 & -0.878380 & 0.000000
\end{tabular}




$\begin{array}{llll}13 \mathrm{C} & -2.385865 & -1.832025 & 0.000000 \\ 14 \mathrm{O} & -3.203896 & 1.545152 & 0.000000 \\ 15 \mathrm{O} & -0.411030 & 2.315255 & 0.000000 \\ 16 \mathrm{H} & 2.773725 & -2.491075 & 0.000000 \\ 17 \mathrm{H} & 4.391709 & 1.487313 & 0.000000 \\ 18 \mathrm{H} & 2.078744 & 2.421583 & 0.000000 \\ 19 \mathrm{H} & 4.725134 & -0.972262 & 0.000000 \\ 20 \mathrm{H} & 0.273479 & -2.832883 & 0.000000 \\ 21 \mathrm{H} & -4.406281 & -0.991340 & 0.000000 \\ 22 \mathrm{H} & -2.525813 & -2.903661 & 0.000000\end{array}$

Point Group: cs Number of degrees of freedom: 41

\section{Modeling Results for 2}
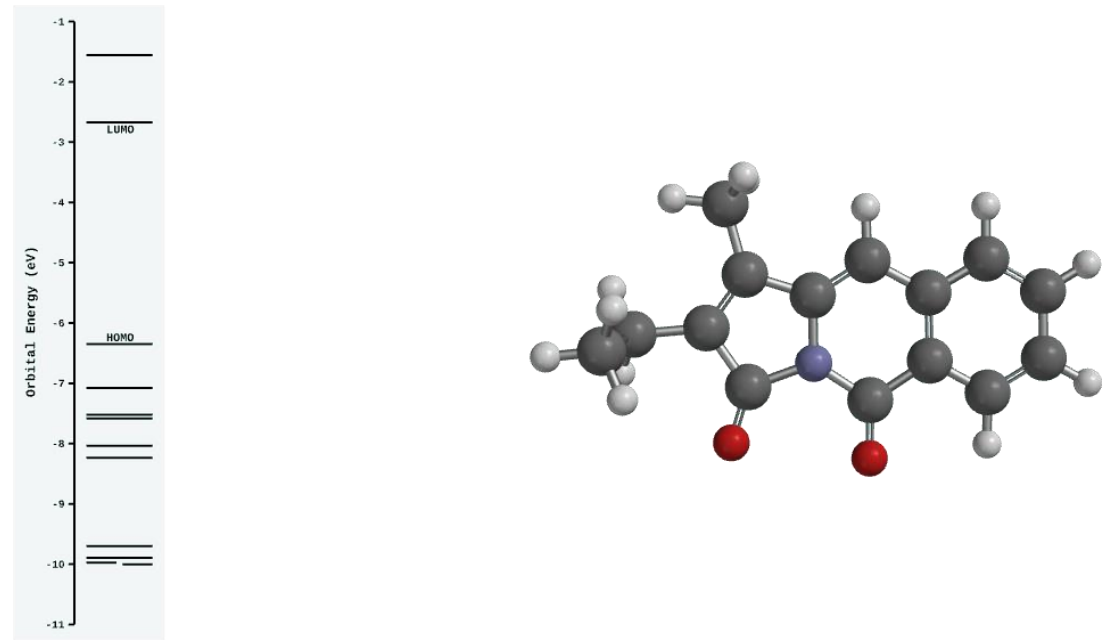

2

MacSPARTAN '14 Quantum Mechanics Program: (x86/Darwin) build 14.119

\section{$\mathrm{C}_{15} \mathrm{H}_{13} \mathrm{NO}_{2}$}

Method: RB3LYP

Basis set: 6-311++G**

Number of shells: 173

Number of basis functions: 487

Multiplicity: 1

Energy: $-784.869021 \mathrm{au}$ 
Coordinates (Angstroms)

\begin{tabular}{llll} 
ATOM & \multicolumn{1}{c}{$\mathrm{X}$} & \multicolumn{1}{c}{$\mathrm{Y}$} & \multicolumn{1}{c}{$\mathrm{Z}$} \\
$1 \mathrm{C}$ & 2.092438 & -0.542879 & -0.009235 \\
2 C & 2.055459 & 0.869320 & 0.055790 \\
$3 \mathrm{C}$ & 0.779410 & 1.541536 & 0.012653 \\
$4 \mathrm{C}$ & -0.356282 & 0.817419 & -0.087016 \\
$5 \mathrm{~N}$ & -0.332533 & -0.581147 & -0.147497 \\
$6 \mathrm{C}$ & 0.848370 & -1.353643 & -0.115276 \\
$7 \mathrm{C}$ & -1.684284 & -1.063203 & -0.259378 \\
8 C & -2.542545 & 0.153760 & -0.256795 \\
$9 \mathrm{C}$ & -1.754003 & 1.249161 & -0.156704 \\
$10 \mathrm{O}$ & 0.837660 & -2.563740 & -0.169327 \\
$11 \mathrm{O}$ & -2.027173 & -2.213009 & -0.345967 \\
$12 \mathrm{C}$ & 3.268144 & 1.574804 & 0.157109 \\
$13 \mathrm{C}$ & 4.477043 & 0.896663 & 0.191026 \\
$14 \mathrm{C}$ & 4.505773 & -0.501644 & 0.124278 \\
$15 \mathrm{C}$ & 3.318683 & -1.214090 & 0.025802 \\
$16 \mathrm{C}$ & -4.030876 & 0.029804 & -0.342409 \\
$17 \mathrm{C}$ & -4.687396 & -0.296566 & 1.012805 \\
$18 \mathrm{C}$ & -2.155295 & 2.689326 & -0.118541 \\
$19 \mathrm{H}$ & 0.735420 & 2.622188 & 0.058085 \\
$20 \mathrm{H}$ & 3.248362 & 2.658193 & 0.208400 \\
$21 \mathrm{H}$ & 5.404292 & 1.453290 & 0.269328 \\
$22 \mathrm{H}$ & 5.453476 & -1.026650 & 0.150111 \\
$23 \mathrm{H}$ & 3.312303 & -2.295868 & -0.026559 \\
$24 \mathrm{H}$ & -4.267223 & -0.770343 & -1.050276 \\
$25 \mathrm{H}$ & -4.456624 & 0.952382 & -0.747156 \\
$26 \mathrm{H}$ & -4.298958 & -1.233705 & 1.417499 \\
$27 \mathrm{H}$ & -5.769103 & -0.402381 & 0.895801 \\
$28 \mathrm{H}$ & -4.499830 & 0.494705 & 1.743495 \\
$29 \mathrm{H}$ & -3.231026 & 2.803046 & -0.251752 \\
$30 \mathrm{H}$ & -1.652545 & 3.259101 & -0.906300 \\
$31 \mathrm{H}$ & -1.882820 & 3.146882 & 0.838078
\end{tabular}

Point Group: c1 Number of degrees of freedom: 87 


\section{Modeling Results for 3}
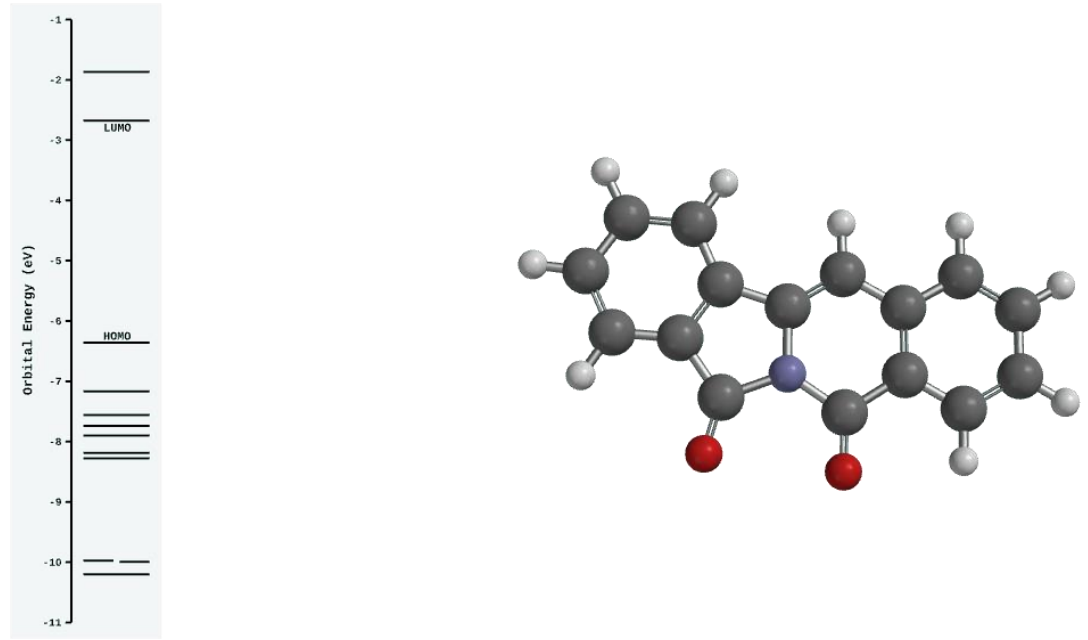

3

MacSPARTAN '14 Quantum Mechanics Program: (x86/Darwin) build 14.119

\section{$\mathrm{C}_{16} \mathrm{H}_{9} \mathrm{NO}_{2}$}

Method: RB3LYP

Basis set: $6-311++\mathrm{G}^{* *}$

Number of shells: 159

Number of basis functions: 481

Multiplicity: 1

Energy: -820.573309 au

Coordinates (Angstroms)

\begin{tabular}{llll} 
ATOM & \multicolumn{1}{c}{$\mathrm{X}$} & \multicolumn{1}{c}{$\mathrm{Y}$} & \multicolumn{1}{c}{$\mathrm{Z}$} \\
1 C & 3.063897 & -1.909240 & 0.000000 \\
2 C & 4.612520 & -0.051933 & 0.000000 \\
3 C & 2.233206 & 0.372200 & 0.000000 \\
4 C & 3.551232 & 0.841433 & 0.000000 \\
5 C & 1.974801 & -1.018373 & 0.000000 \\
6 C & 4.364366 & -1.430919 & 0.000000 \\
7 C & 0.609852 & -1.480249 & 0.000000 \\
8 C & -0.401568 & -0.587480 & 0.000000 \\
9 N & -0.164462 & 0.799699 & 0.000000 \\
10 C & 1.132748 & 1.371474 & 0.000000 \\
11 C & -1.412163 & 1.530608 & 0.000000 \\
12 C & -2.457878 & 0.477577 & 0.000000 \\
13 C & -1.850242 & -0.780789 & 0.000000 \\
14 C & -2.627568 & -1.937547 & 0.000000
\end{tabular}




$\begin{array}{llll}15 \mathrm{C} & -4.014168 & -1.794444 & 0.000000 \\ 16 \mathrm{C} & -4.617567 & -0.528949 & 0.000000 \\ 17 \mathrm{C} & -3.839478 & 0.626628 & 0.000000 \\ 18 \mathrm{O} & -1.541222 & 2.723809 & 0.000000 \\ 19 \mathrm{O} & 1.311128 & 2.568993 & 0.000000 \\ 20 \mathrm{H} & 2.874859 & -2.977286 & 0.000000 \\ 21 \mathrm{H} & 5.632016 & 0.315864 & 0.000000 \\ 22 \mathrm{H} & 3.712922 & 1.912259 & 0.000000 \\ 23 \mathrm{H} & 5.194265 & -2.128849 & 0.000000 \\ 24 \mathrm{H} & 0.397654 & -2.541729 & 0.000000 \\ 25 \mathrm{H} & -2.177823 & -2.923649 & 0.000000 \\ 26 \mathrm{H} & -4.639748 & -2.679952 & 0.000000 \\ 27 \mathrm{H} & -5.698622 & -0.453490 & 0.000000 \\ 28 \mathrm{H} & -4.284289 & 1.614735 & 0.000000\end{array}$

Point Group: cs Number of degrees of freedom: 53

\section{Modeling Results for 4}
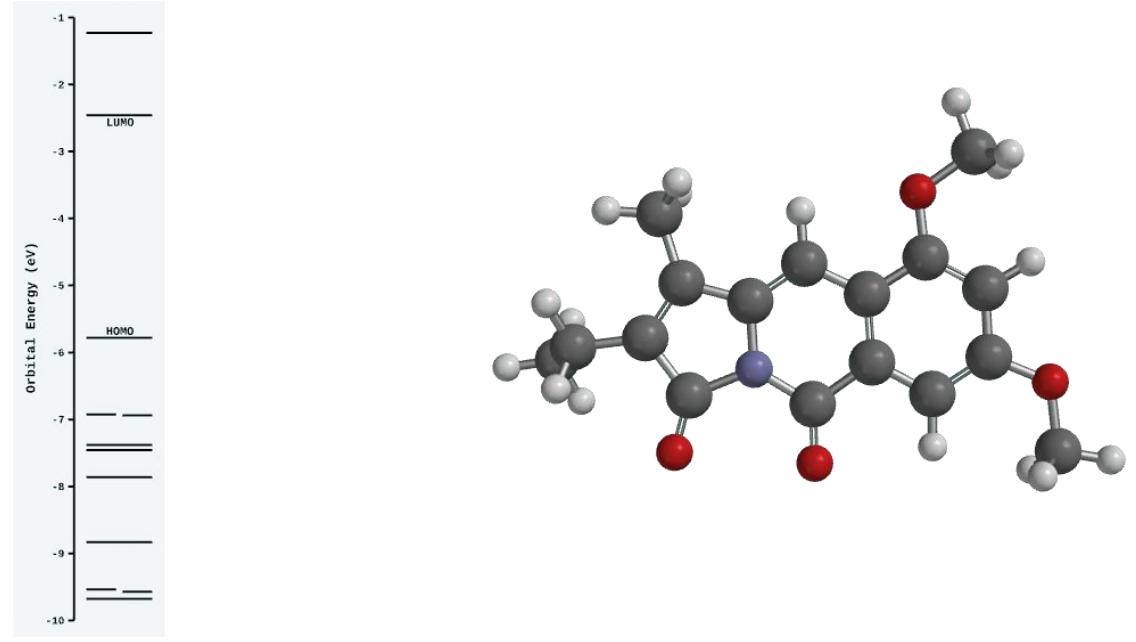

\section{4}

MacSPARTAN '14 Quantum Mechanics Program: (x86/Darwin) build 14.119

\section{$\mathrm{C}_{17} \mathrm{H}_{17} \mathrm{NO}_{4}$}

Method: RB3LYP

Basis set: $6-311++\mathrm{G}^{* *}$

Number of shells: 217

Number of basis functions: 603

Multiplicity: 1 
Energy: -1013.98451 au

Coordinates (Angstroms)

\begin{tabular}{llll} 
ATOM & \multicolumn{1}{c}{$\mathrm{X}$} & \multicolumn{1}{c}{$\mathrm{Y}$} & \multicolumn{1}{c}{$\mathrm{Z}$} \\
1 C & 2.314599 & -1.390332 & 0.059829 \\
2 C & 3.500774 & 0.718763 & 0.049606 \\
3 C & 1.100092 & 0.703125 & -0.044792 \\
4 C & 2.303086 & 1.417495 & -0.015744 \\
5 C & 1.072238 & -0.702674 & -0.009337 \\
6 C & 3.506785 & -0.688673 & 0.088813 \\
7 C & -0.185894 & -1.396894 & -0.047536 \\
$8 \mathrm{C}$ & -1.334654 & -0.687374 & -0.115597 \\
9 N & -1.328341 & 0.713273 & -0.146795 \\
$10 \mathrm{C}$ & -0.161182 & 1.496529 & -0.115686 \\
$11 \mathrm{C}$ & -2.689361 & 1.181889 & -0.230387 \\
$12 \mathrm{C}$ & -3.529974 & -0.043054 & -0.242352 \\
$13 \mathrm{C}$ & -2.725348 & -1.131529 & -0.175045 \\
$14 \mathrm{O}$ & -3.044287 & 2.331072 & -0.287432 \\
$15 \mathrm{O}$ & -0.181945 & 2.709215 & -0.143875 \\
$16 \mathrm{C}$ & -5.021309 & 0.062825 & -0.306543 \\
$17 \mathrm{C}$ & -5.667232 & 0.345577 & 1.063301 \\
$18 \mathrm{C}$ & -3.111326 & -2.576390 & -0.160421 \\
$19 \mathrm{O}$ & 2.229431 & -2.748556 & 0.092788 \\
$20 \mathrm{C}$ & 3.429391 & -3.511329 & 0.157067 \\
$21 \mathrm{O}$ & 4.731221 & 1.296223 & 0.081522 \\
$22 \mathrm{C}$ & 4.812153 & 2.719199 & 0.044970 \\
$23 \mathrm{H}$ & 2.251018 & 2.495960 & -0.046127 \\
$24 \mathrm{H}$ & 4.464649 & -1.186234 & 0.142208 \\
$25 \mathrm{H}$ & -0.204794 & -2.476666 & -0.024530 \\
$26 \mathrm{H}$ & -5.276960 & 0.877360 & -0.991111 \\
$27 \mathrm{H}$ & -5.440609 & -0.854488 & -0.729868 \\
$28 \mathrm{H}$ & -5.281732 & 1.274238 & 1.489647 \\
$29 \mathrm{H}$ & -6.751166 & 0.445477 & 0.961040 \\
$30 \mathrm{H}$ & -5.465593 & -0.464112 & 1.769939 \\
$31 \mathrm{H}$ & -4.186022 & -2.699966 & -0.293797 \\
$32 \mathrm{H}$ & -2.831578 & -3.046454 & 0.787830 \\
$33 \mathrm{H}$ & -2.602811 & -3.127546 & -0.957300 \\
$34 \mathrm{H}$ & 3.116352 & -4.553862 & 0.172189 \\
$35 \mathrm{H}$ & 3.994208 & -3.287843 & 1.067969 \\
$36 \mathrm{H}$ & 4.059973 & -3.333012 & -0.719918 \\
$37 \mathrm{H}$ & 5.874259 & 2.954676 & 0.078813 \\
$38 \mathrm{H}$ & 4.309706 & 3.166219 & 0.908241 \\
$39 \mathrm{H}$ & 4.377798 & 3.116441 & -0.877614
\end{tabular}

Point Group: c1 Number of degrees of freedom: 111 


\section{Modeling Results for 5}
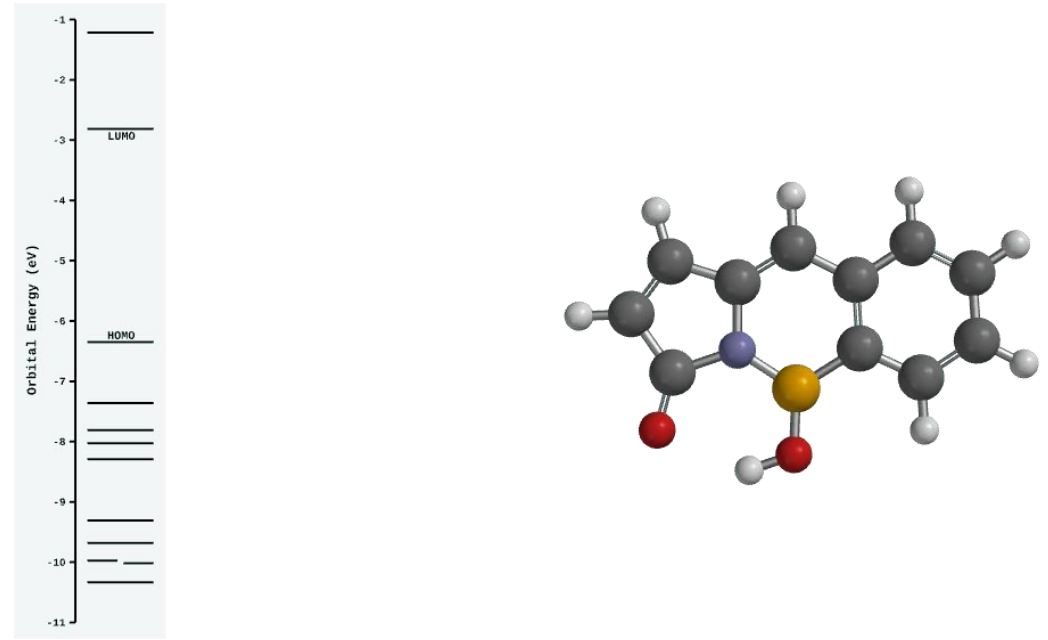

5

MacSPARTAN '14 Quantum Mechanics Program: (x86/Darwin) build 14.119

\section{$\mathrm{C}_{11} \mathrm{H}_{8} \mathrm{BNO}_{2}$}

Method: RB3LYP

Basis set: 6-311++G**

Number of shells: 130

Number of basis functions: 386

Multiplicity: 1

Energy: -654.338059 au

Coordinates (Angstroms)

\begin{tabular}{llll} 
ATOM & \multicolumn{1}{c}{$\mathrm{X}$} & \multicolumn{1}{c}{$\mathrm{Y}$} & \multicolumn{1}{c}{$\mathrm{Z}$} \\
1 C & -0.198117 & -1.741048 & 0.000000 \\
2 C & -1.189535 & 0.542673 & 0.000000 \\
3 N & 1.309839 & 0.131525 & 0.000000 \\
4 B & 0.249206 & 1.125986 & 0.000000 \\
5 C & 1.062135 & -1.244571 & 0.000000 \\
6 C & -1.358491 & -0.870508 & 0.000000 \\
7 C & -2.655661 & -1.414649 & 0.000000 \\
8 C & -3.767484 & -0.583995 & 0.000000 \\
9 C & -3.607644 & 0.805296 & 0.000000 \\
10 C & -2.329581 & 1.356151 & 0.000000 \\
11 C & 2.355210 & -1.911613 & 0.000000 \\
12 C & 3.332761 & -0.980349 & 0.000000 \\
13 C & 2.702301 & 0.356346 & 0.000000 \\
14 O & 0.534171 & 2.452193 & 0.000000
\end{tabular}




$\begin{array}{llll}15 \mathrm{O} & 3.212051 & 1.465369 & 0.000000 \\ 16 \mathrm{H} & -0.341851 & -2.815525 & 0.000000 \\ 17 \mathrm{H} & -2.782473 & -2.492464 & 0.000000 \\ 18 \mathrm{H} & -4.762919 & -1.014362 & 0.000000 \\ 19 \mathrm{H} & -4.479236 & 1.450242 & 0.000000 \\ 20 \mathrm{H} & -2.203824 & 2.433420 & 0.000000 \\ 21 \mathrm{H} & 2.472534 & -2.986273 & 0.000000 \\ 22 \mathrm{H} & 4.402027 & -1.124622 & 0.000000 \\ 23 \mathrm{H} & 1.493200 & 2.607909 & 0.000000\end{array}$

Point Group: cs Number of degrees of freedom: 43

\section{Modeling Results for 6}
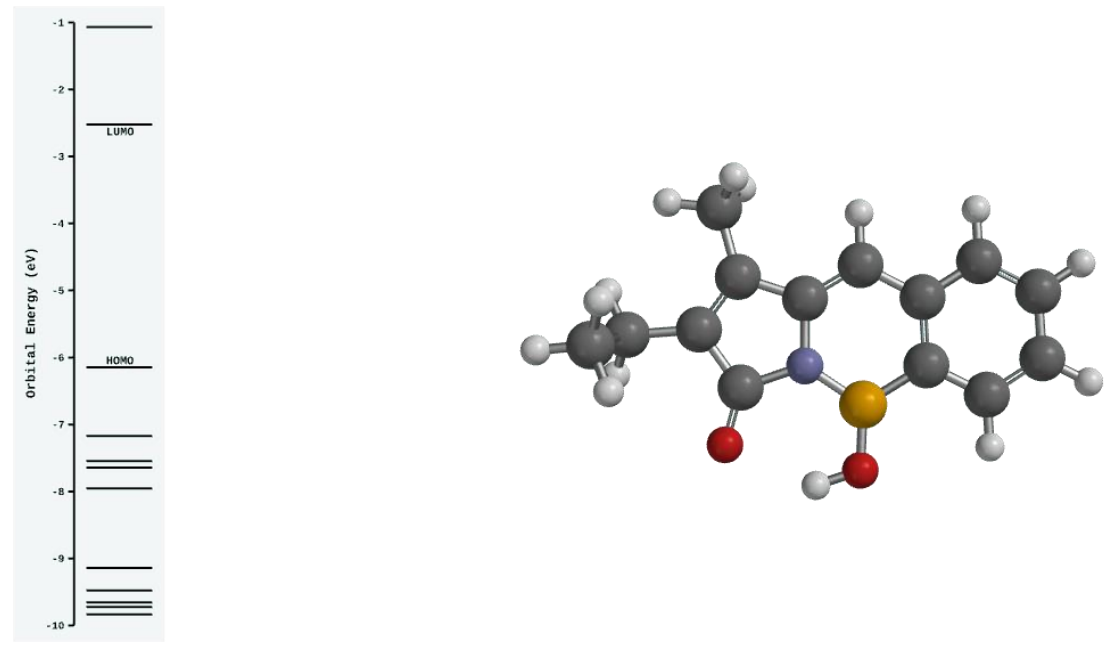

6

MacSPARTAN '14 Quantum Mechanics Program: (x86/Darwin) build 14.119

\section{$\mathrm{C}_{14} \mathrm{H}_{14} \mathrm{BNO}_{2}$}

Method: RB3LYP

Basis set: $6-311++\mathrm{G}^{* *}$

Number of shells: 178

Number of basis functions: 494

Multiplicity: 1

Energy: -772.324406 au

Coordinates (Angstroms)

\begin{tabular}{llll} 
ATOM & \multicolumn{1}{c}{ X } & \multicolumn{1}{c}{ Y } & Z \\
$1 \mathrm{C}$ & -0.798021 & -1.518387 & 0.010197 \\
2 C & -2.154998 & 0.572021 & -0.006167 \\
3 N & 0.371586 & 0.569494 & -0.143906
\end{tabular}




$\begin{array}{llll}\text { 4 B } & -0.834764 & 1.377746 & -0.114560 \\ \text { 5 C } & 0.362114 & -0.827762 & -0.085735 \\ 6 \mathrm{C} & -2.086081 & -0.847480 & 0.054502 \\ 7 \mathrm{C} & -3.274318 & -1.593797 & 0.153962 \\ 8 \mathrm{C} & -4.506740 & -0.956203 & 0.191684 \\ 9 \mathrm{C} & -4.580498 & 0.438980 & 0.130325 \\ 10 \mathrm{C} & -3.412471 & 1.188569 & 0.033194 \\ 11 \mathrm{C} & 1.754183 & -1.284338 & -0.154859 \\ 12 \mathrm{C} & 2.561541 & -0.195193 & -0.252318 \\ 13 \mathrm{C} & 1.700664 & 1.011788 & -0.248049 \\ 14 \mathrm{O} & -0.769853 & 2.732748 & -0.179306 \\ 15 \mathrm{O} & 2.023586 & 2.188580 & -0.326294 \\ 16 \mathrm{C} & 4.051910 & -0.091146 & -0.339094 \\ 17 \mathrm{C} & 4.715374 & 0.235344 & 1.012360 \\ 18 \mathrm{C} & 2.142728 & -2.727792 & -0.120272 \\ 19 \mathrm{H} & -0.772228 & -2.601006 & 0.053540 \\ 20 \mathrm{H} & -3.222371 & -2.676917 & 0.201382 \\ 21 \mathrm{H} & -5.415203 & -1.543658 & 0.268610 \\ 22 \mathrm{H} & -5.545575 & 0.932275 & 0.158980 \\ 23 \mathrm{H} & -3.465754 & 2.270878 & -0.014453 \\ 24 \mathrm{H} & 0.150320 & 3.037355 & -0.243273 \\ 25 \mathrm{H} & 4.466157 & -1.021543 & -0.738179 \\ 26 \mathrm{H} & 4.300172 & 0.699828 & -1.053729 \\ 27 \mathrm{H} & 4.518301 & -0.548745 & 1.748485 \\ 28 \mathrm{H} & 5.798423 & 0.326984 & 0.894515 \\ 29 \mathrm{H} & 4.338692 & 1.179543 & 1.412054 \\ 30 \mathrm{H} & 1.872650 & -3.182101 & 0.838459 \\ 31 \mathrm{H} & 3.216130 & -2.854122 & -0.262140 \\ 32 \mathrm{H} & 1.626696 & -3.292383 & -0.902826\end{array}$

Point Group: c1 Number of degrees of freedom: 90 


\section{Modeling Results for 7}
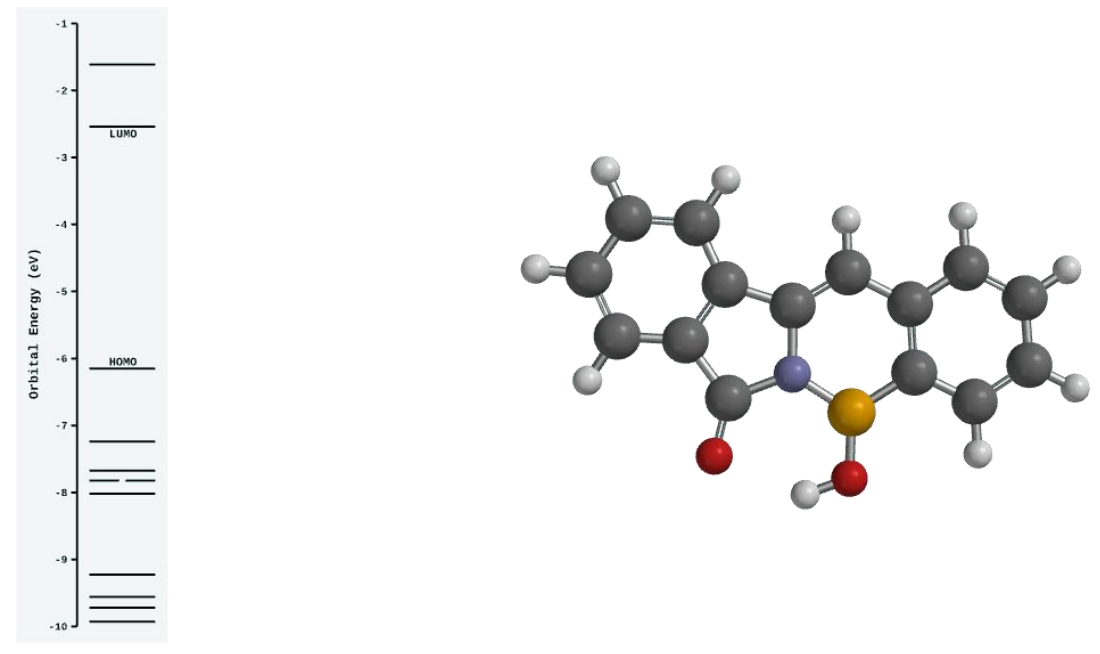

7

MacSPARTAN '14 Quantum Mechanics Program: (x86/Darwin) build 14.119

\section{$\mathrm{C}_{15} \mathrm{H}_{10} \mathrm{BNO}{ }_{2}$}

Method: RB3LYP

Basis set: $6-311++\mathrm{G}^{* *}$

Number of shells: 164

Number of basis functions: 488

Multiplicity: 1

Energy: -808.029224 au

Coordinates (Angstroms)

\begin{tabular}{llll} 
ATOM & \multicolumn{1}{c}{$\mathrm{X}$} & \multicolumn{1}{c}{$\mathrm{Y}$} & \multicolumn{1}{c}{$\mathrm{Z}$} \\
1 C & -0.624939 & -1.459136 & 0.000000 \\
2 C & -2.298530 & 0.385337 & 0.000000 \\
3 N & 0.205302 & 0.798449 & 0.000000 \\
4 B & -1.126346 & 1.396655 & 0.000000 \\
5 C & 0.412560 & -0.593124 & 0.000000 \\
6 C & -2.003270 & -1.006048 & 0.000000 \\
7 C & -3.058513 & -1.936592 & 0.000000 \\
8 C & -4.377466 & -1.506650 & 0.000000 \\
9 C & -4.673892 & -0.139178 & 0.000000 \\
10 C & -3.640217 & 0.790893 & 0.000000 \\
11 C & 1.860350 & -0.809670 & 0.000000 \\
12 C & 2.484951 & 0.447025 & 0.000000 \\
13 C & 1.432953 & 1.486622 & 0.000000 \\
14 O & -1.285096 & 2.744244 & 0.000000 \\
15 O & 1.537266 & 2.702048 & 0.000000 \\
16 C & 3.868430 & 0.580969 & 0.000000
\end{tabular}




$\begin{array}{llll}17 \mathrm{C} & 2.629927 & -1.971906 & 0.000000 \\ 18 \mathrm{C} & 4.634801 & -0.582365 & 0.000000 \\ 19 \mathrm{C} & 4.017684 & -1.841627 & 0.000000 \\ 20 \mathrm{H} & -0.424913 & -2.524265 & 0.000000 \\ 21 \mathrm{H} & -2.834323 & -2.998442 & 0.000000 \\ 22 \mathrm{H} & -5.181415 & -2.234755 & 0.000000 \\ 23 \mathrm{H} & -5.706416 & 0.191880 & 0.000000 \\ 24 \mathrm{H} & -3.865241 & 1.851942 & 0.000000 \\ 25 \mathrm{H} & -0.429009 & 3.203515 & 0.000000 \\ 26 \mathrm{H} & 4.325661 & 1.563464 & 0.000000 \\ 27 \mathrm{H} & 2.171467 & -2.954052 & 0.000000 \\ 28 \mathrm{H} & 5.716610 & -0.518163 & 0.000000 \\ 29 \mathrm{H} & 4.634630 & -2.733289 & 0.000000\end{array}$

Point Group: cs Number of degrees of freedom: 55

\section{Modeling Results for 8}
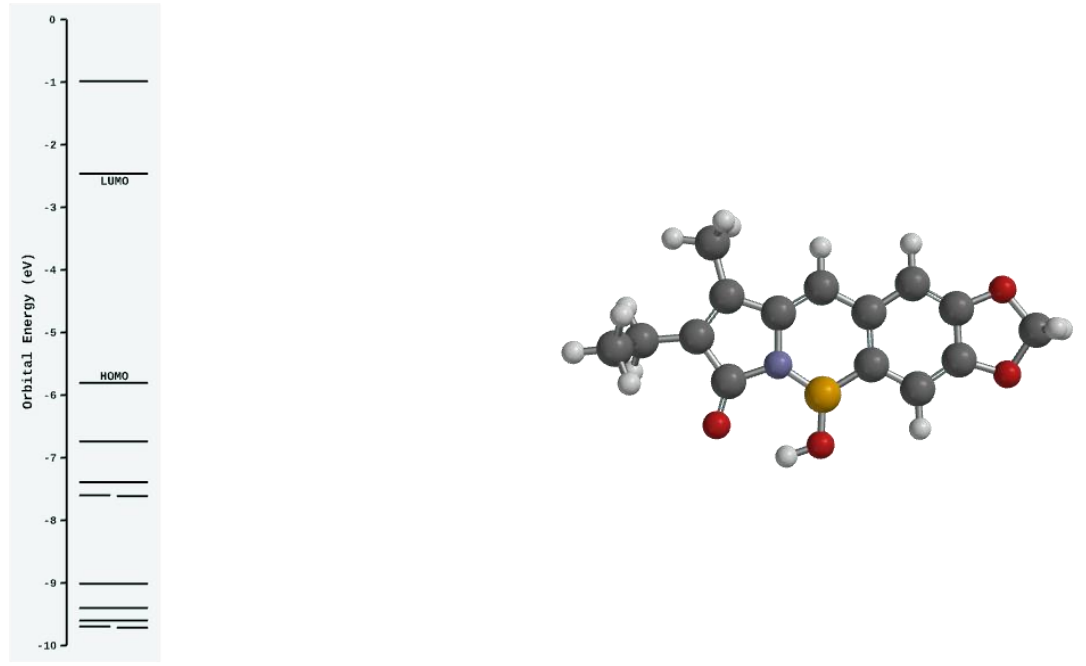

8

MacSPARTAN '14 Quantum Mechanics Program: (x86/Darwin) build 14.119

\section{$\mathrm{C}_{15} \mathrm{H}_{14} \mathrm{BNO}_{4}$}

Method: RB3LYP

Basis set: $6-311++\mathrm{G}^{* *}$

Number of shells: 196

Number of basis functions: 560

Multiplicity: 1

Energy: -960.907379 au 


\begin{tabular}{|c|c|c|c|}
\hline \multicolumn{4}{|c|}{ Coordinates (Angstroms) } \\
\hline ATOM & $\mathrm{X}$ & Y & $\mathrm{Z}$ \\
\hline $1 \mathrm{C}$ & 2.397331 & -1.518356 & -0.032198 \\
\hline $2 \mathrm{C}$ & 2.464998 & 1.331053 & 0.084520 \\
\hline $3 \mathrm{C}$ & 1.195311 & -0.770565 & 0.036781 \\
\hline $4 \mathrm{C}$ & 3.576452 & -0.816094 & -0.045208 \\
\hline $5 \mathrm{C}$ & 3.612682 & 0.578617 & 0.012423 \\
\hline $6 \mathrm{C}$ & 1.228450 & 0.650655 & 0.093757 \\
\hline $7 \mathrm{O}$ & 4.920635 & 0.998553 & 0.025604 \\
\hline $8 \mathrm{C}$ & 5.695803 & -0.162190 & -0.289405 \\
\hline 90 & 4.856347 & -1.306363 & -0.074276 \\
\hline $10 \mathrm{~B}$ & -0.112420 & 1.420409 & 0.163480 \\
\hline $11 \mathrm{~N}$ & -1.300785 & 0.586752 & 0.165292 \\
\hline $12 \mathrm{C}$ & -1.257600 & -0.808333 & 0.113569 \\
\hline $13 \mathrm{C}$ & -0.076829 & -1.468985 & 0.050247 \\
\hline $14 \mathrm{C}$ & -2.638636 & -1.297652 & 0.149230 \\
\hline $15 \mathrm{C}$ & -3.474231 & -0.227160 & 0.221015 \\
\hline $16 \mathrm{C}$ & -2.642468 & 0.999157 & 0.233029 \\
\hline $17 \mathrm{O}$ & -0.207252 & 2.775285 & 0.219951 \\
\hline $18 \mathrm{O}$ & -2.993348 & 2.168936 & 0.297332 \\
\hline $19 \mathrm{C}$ & -4.968443 & -0.157779 & 0.269900 \\
\hline $20 \mathrm{C}$ & -5.607797 & 0.125603 & -1.102820 \\
\hline $21 \mathrm{C}$ & -2.992776 & -2.749828 & 0.112595 \\
\hline $22 \mathrm{H}$ & 2.388210 & -2.600835 & -0.071122 \\
\hline $23 \mathrm{H}$ & 2.500140 & 2.412232 & 0.135902 \\
\hline $24 \mathrm{H}$ & 5.993937 & -0.127206 & -1.344351 \\
\hline $25 \mathrm{H}$ & 6.557707 & -0.215850 & 0.374305 \\
\hline $26 \mathrm{H}$ & -0.075090 & -2.552003 & 0.010979 \\
\hline $27 \mathrm{H}$ & -1.134656 & 3.061156 & 0.260214 \\
\hline $28 \mathrm{H}$ & -5.370780 & -1.089476 & 0.678229 \\
\hline $29 \mathrm{H}$ & -5.251993 & 0.641175 & 0.962149 \\
\hline $30 \mathrm{H}$ & -6.694775 & 0.199565 & -1.011010 \\
\hline $31 \mathrm{H}$ & -5.239584 & 1.067459 & -1.515634 \\
\hline $32 \mathrm{H}$ & -5.380524 & -0.670961 & -1.816591 \\
\hline $33 \mathrm{H}$ & -2.694934 & -3.201313 & -0.839308 \\
\hline $34 \mathrm{H}$ & -4.065407 & -2.900843 & 0.235111 \\
\hline $35 \mathrm{H}$ & -2.478455 & -3.299722 & 0.906612 \\
\hline
\end{tabular}

Point Group: c1 Number of degrees of freedom: 99 


\section{Modeling Results for 9}
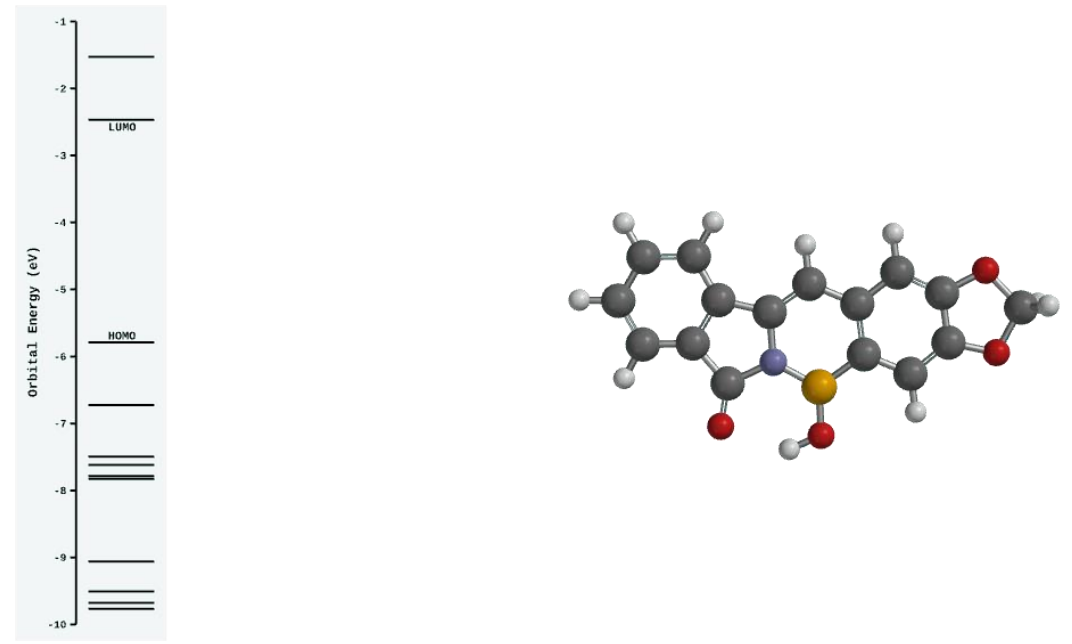

9

MacSPARTAN '14 Quantum Mechanics Program: (x86/Darwin) build 14.119

\section{$\mathrm{C}_{16} \mathrm{H}_{10} \mathrm{BNO} \mathrm{H}_{4}$}

Method: RB3LYP

Basis set: $6-311++\mathrm{G}^{* *}$

Number of shells: 182

Number of basis functions: 554

Multiplicity: 1

Energy: -996.612062 au

Coordinates (Angstroms)

\begin{tabular}{llll} 
ATOM & \multicolumn{1}{c}{$\mathrm{X}$} & \multicolumn{1}{c}{$\mathrm{Y}$} & \multicolumn{1}{c}{$\mathrm{Z}$} \\
1 O & 4.769957 & -1.620551 & 0.001040 \\
2 C & 5.752851 & -0.572131 & -0.002255 \\
3 O & 5.055515 & 0.679395 & -0.001267 \\
4 C & 3.716508 & 0.380185 & -0.000106 \\
5 C & 3.547849 & -1.005614 & 0.000909 \\
6 C & 2.307697 & -1.592634 & 0.001904 \\
7 C & 1.180980 & -0.732721 & 0.001854 \\
8 C & 1.348510 & 0.679738 & 0.001389 \\
9 C & 2.645200 & 1.239868 & 0.000102 \\
$10 \mathrm{~B}$ & 0.090179 & 1.575917 & 0.001217 \\
$11 \mathrm{~N}$ & -1.182816 & 0.862161 & 0.001511 \\
$12 \mathrm{C}$ & -1.264913 & -0.541104 & 0.001413 \\
$13 \mathrm{C}$ & -0.150135 & -1.306583 & 0.001564 \\
$14 \mathrm{C}$ & -2.686570 & -0.887645 & 0.000721 \\
15 C & -3.421254 & 0.308535 & 0.000582 \\
$16 \mathrm{C}$ & -2.466813 & 1.437766 & 0.000400
\end{tabular}




$\begin{array}{llll}17 \mathrm{O} & -2.679632 & 2.639599 & -0.000807 \\ 18 \mathrm{O} & 0.126087 & 2.934268 & -0.000105 \\ 19 \mathrm{C} & -4.810756 & 0.318633 & -0.000138 \\ 20 \mathrm{C} & -5.471158 & -0.908247 & -0.001112 \\ 21 \mathrm{C} & -4.744496 & -2.107465 & -0.001420 \\ 22 \mathrm{C} & -3.350729 & -2.113706 & -0.000424 \\ 23 \mathrm{H} & 6.360274 & -0.650716 & -0.907648 \\ 24 \mathrm{H} & 6.365281 & -0.649242 & 0.899795 \\ 25 \mathrm{H} & 2.196916 & -2.670070 & 0.002478 \\ 26 \mathrm{H} & 2.781134 & 2.314092 & -0.000570 \\ 27 \mathrm{H} & -0.252037 & -2.385452 & 0.001035 \\ 28 \mathrm{H} & -0.769280 & 3.311572 & -0.001316 \\ 29 \mathrm{H} & -5.353698 & 1.256476 & -0.000280 \\ 30 \mathrm{H} & -6.554377 & -0.940421 & -0.001950 \\ 31 \mathrm{H} & -5.279603 & -3.050458 & -0.002623 \\ 32 \mathrm{H} & -2.806326 & -3.050977 & -0.000896\end{array}$

Point Group: c1 Number of degrees of freedom: 90

\section{Modeling Results for 10}
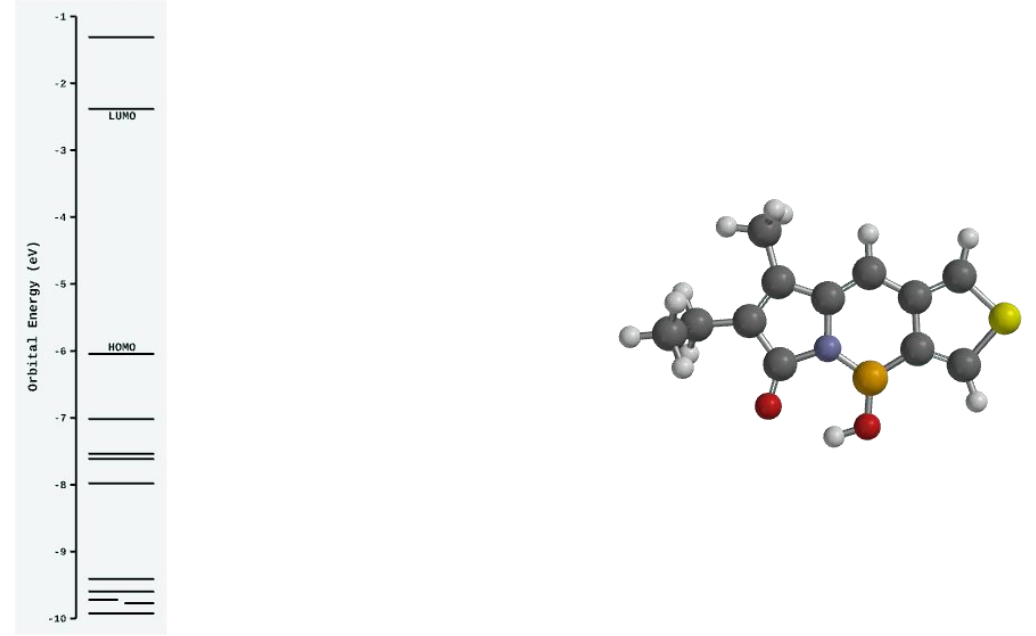

10

MacSPARTAN '14 Quantum Mechanics Program: (x86/Darwin) build 14.119

\section{$\mathrm{C}_{12} \mathrm{H}_{12} \mathrm{BNO}_{2} \mathrm{~S}$}

Method: RB3LYP

Basis set: $6-311++\mathrm{G}^{* *}$

Number of shells: 169

Number of basis functions: 466

Multiplicity: 1 


\section{Energy: -1093.08701 au}

\begin{tabular}{llll}
\multicolumn{4}{l}{ Coordinates (Angstroms) } \\
ATOM & \multicolumn{1}{l}{ X } & \multicolumn{1}{c}{ Y } & \multicolumn{1}{c}{$\mathrm{Z}$} \\
1 C & 3.250403 & -1.439222 & -0.143550 \\
2 S & 4.508554 & -0.255728 & -0.161586 \\
3 C & 3.368654 & 1.041044 & -0.036948 \\
4 C & 2.076005 & 0.592981 & 0.015974 \\
5 C & 2.007951 & -0.854208 & -0.046049 \\
6 C & 0.733584 & -1.538619 & -0.003075 \\
7 C & -0.420017 & -0.835590 & 0.092304 \\
8 N & -0.431759 & 0.569047 & 0.152545 \\
9 B & 0.762507 & 1.402141 & 0.126128 \\
$10 \mathrm{C}$ & -1.760737 & 1.005764 & 0.253339 \\
$11 \mathrm{C}$ & -2.619090 & -0.201148 & 0.253262 \\
$12 \mathrm{C}$ & -1.810289 & -1.290154 & 0.156893 \\
$13 \mathrm{O}$ & -2.089190 & 2.182787 & 0.332375 \\
$14 \mathrm{O}$ & 0.676388 & 2.752884 & 0.193597 \\
$15 \mathrm{C}$ & -4.109880 & -0.099496 & 0.334020 \\
$16 \mathrm{C}$ & -4.768833 & 0.216867 & -1.022071 \\
$17 \mathrm{C}$ & -2.200592 & -2.733484 & 0.117946 \\
$18 \mathrm{H}$ & 3.492940 & -2.489438 & -0.206292 \\
$19 \mathrm{H}$ & 3.719880 & 2.061973 & -0.010279 \\
20 H & 0.701909 & -2.620446 & -0.046602 \\
21 H & -0.251177 & 3.038611 & 0.257572 \\
22 H & -4.524297 & -1.027999 & 0.737470 \\
23 H & -4.361858 & 0.695948 & 1.042264 \\
24 H & -4.568679 & -0.572204 & -1.751963 \\
25 H & -5.852399 & 0.308501 & -0.908858 \\
26 H & -4.391236 & 1.158473 & -1.426907 \\
27 H & -3.273911 & -2.858478 & 0.261614 \\
28 H & -1.684513 & -3.302008 & 0.897550 \\
29 H & -1.934022 & -3.184831 & -0.843165
\end{tabular}

Point Group: c1 Number of degrees of freedom: 81 
Simulated UV-vis spectra of compounds $1-10$ and 20,21 in the gas phase from TD-B3LYP/6-31G(d')
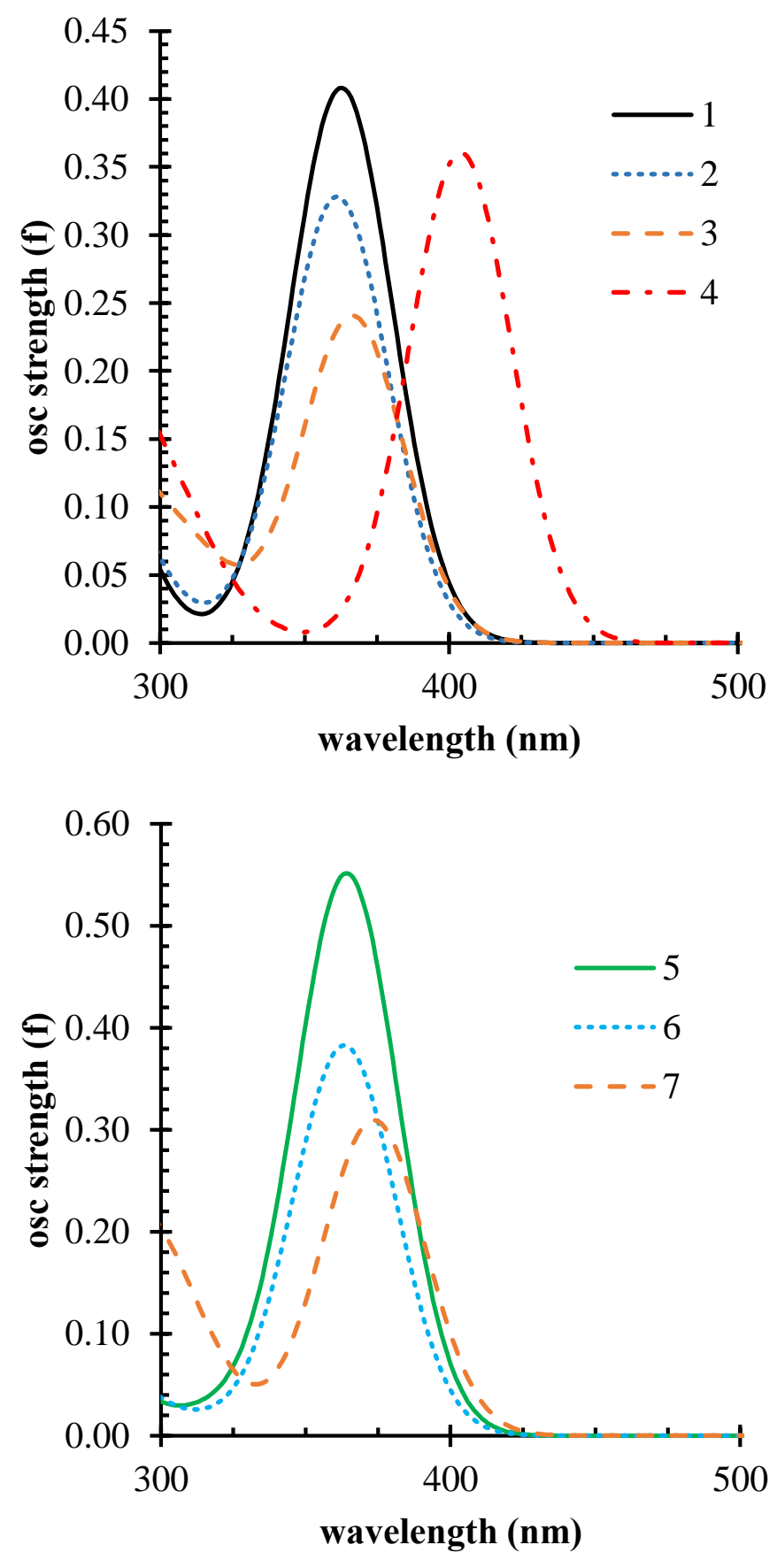

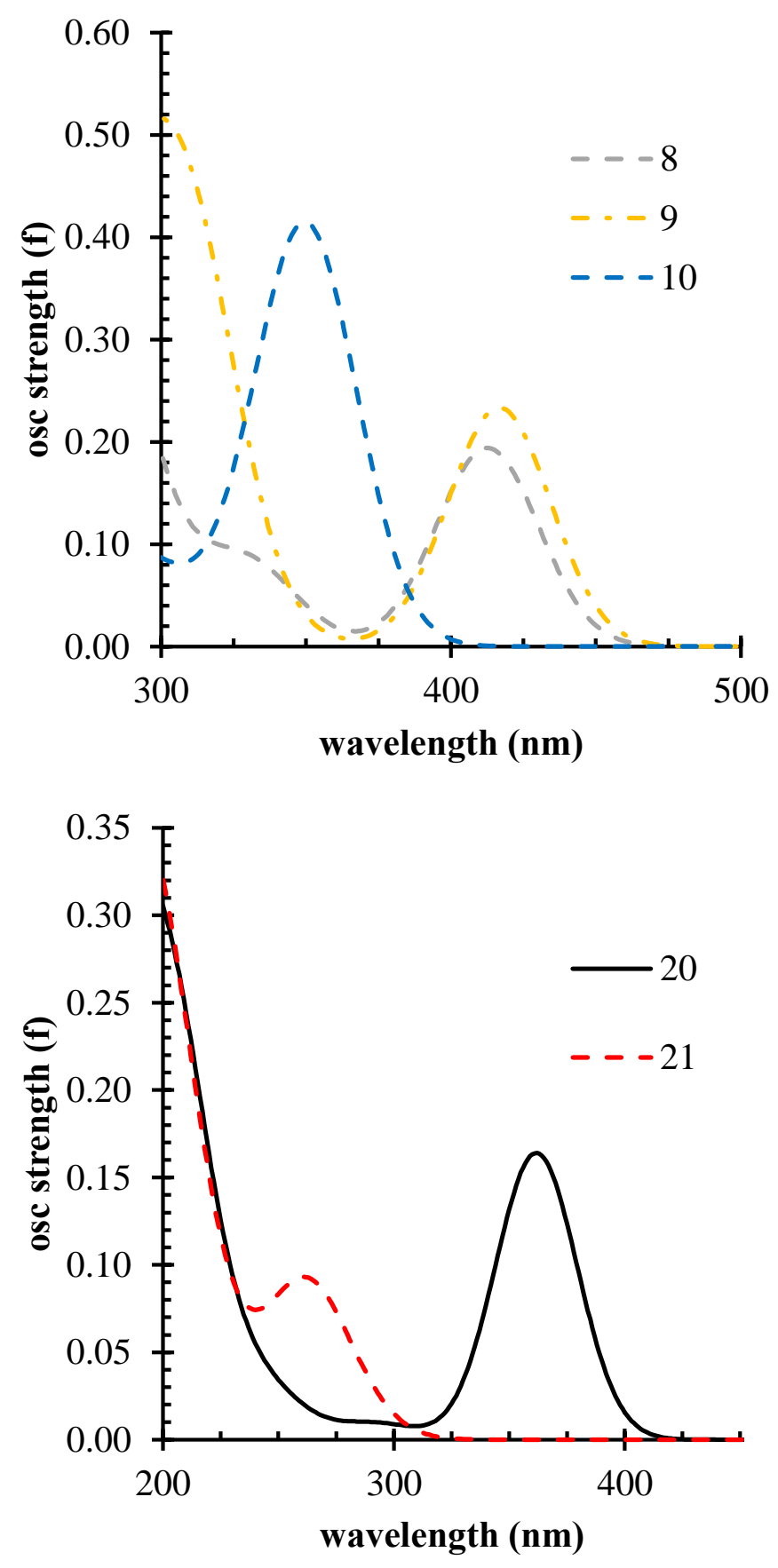


\section{Simulated UV-vis (absorption) and emission spectra of compounds 1, 3-10 in the gas phase from TD-B3LYP/6-31G(d')}
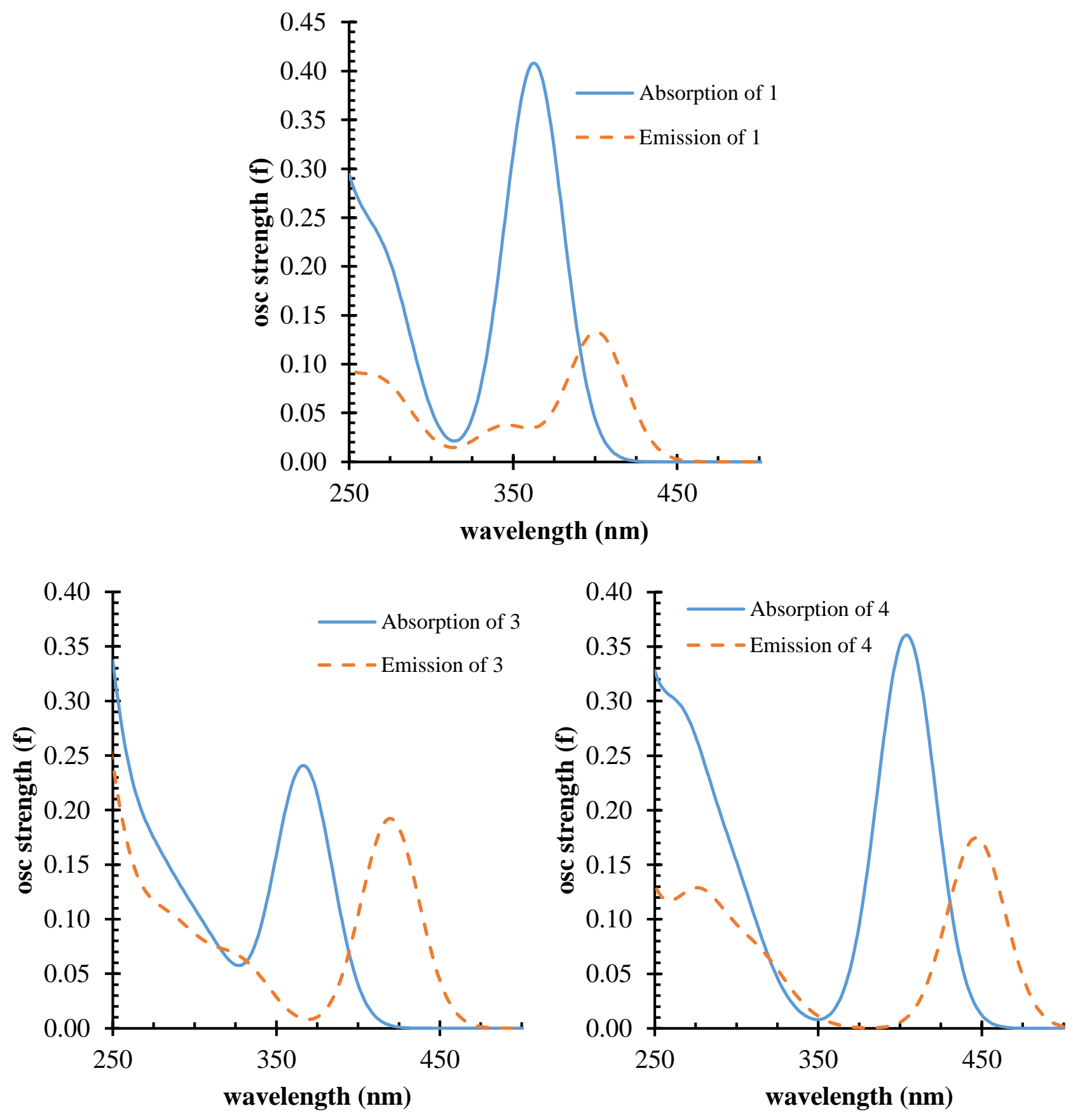

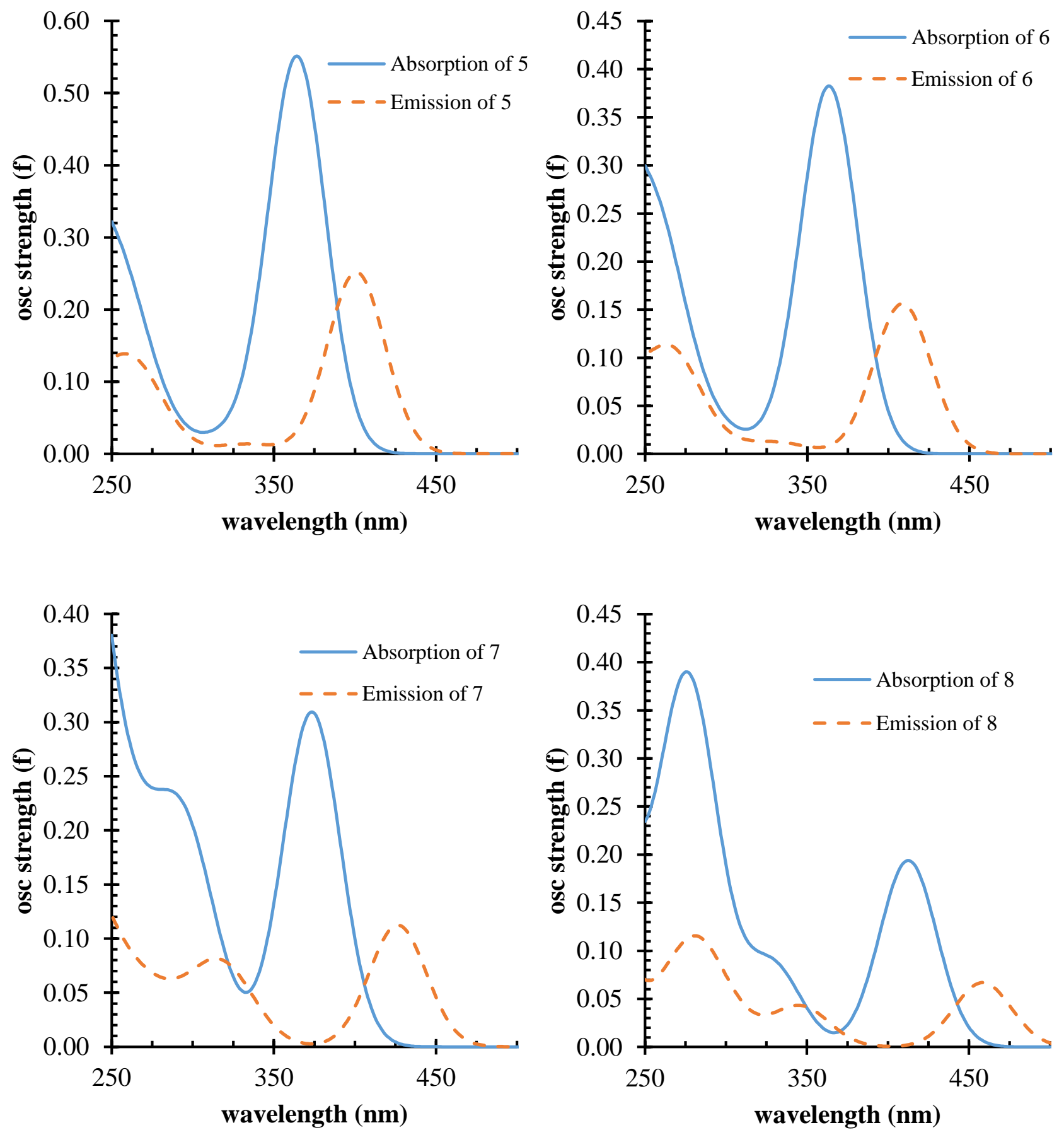

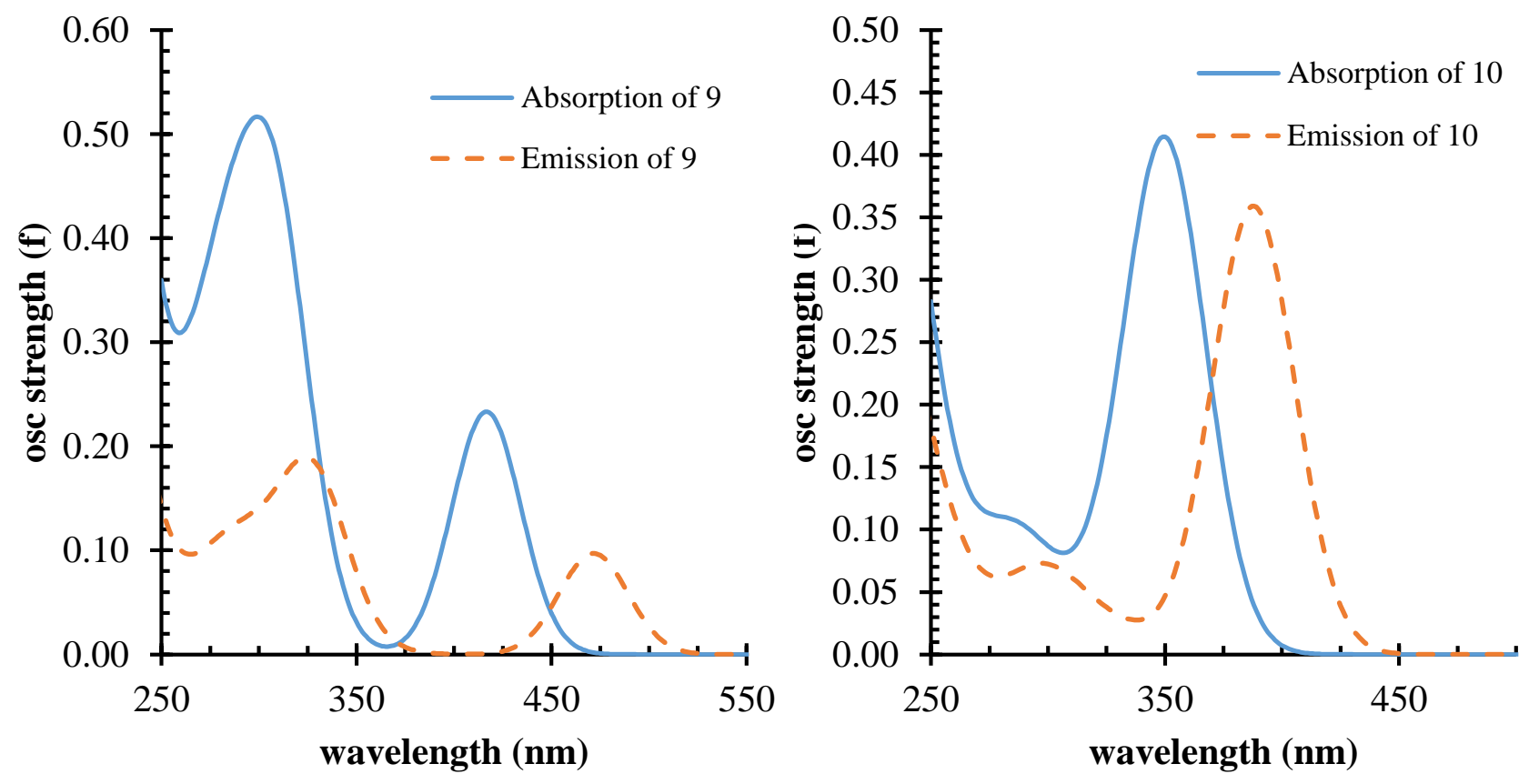


\section{B3LYP/6-31G(d') coordinates for compounds 1-10}

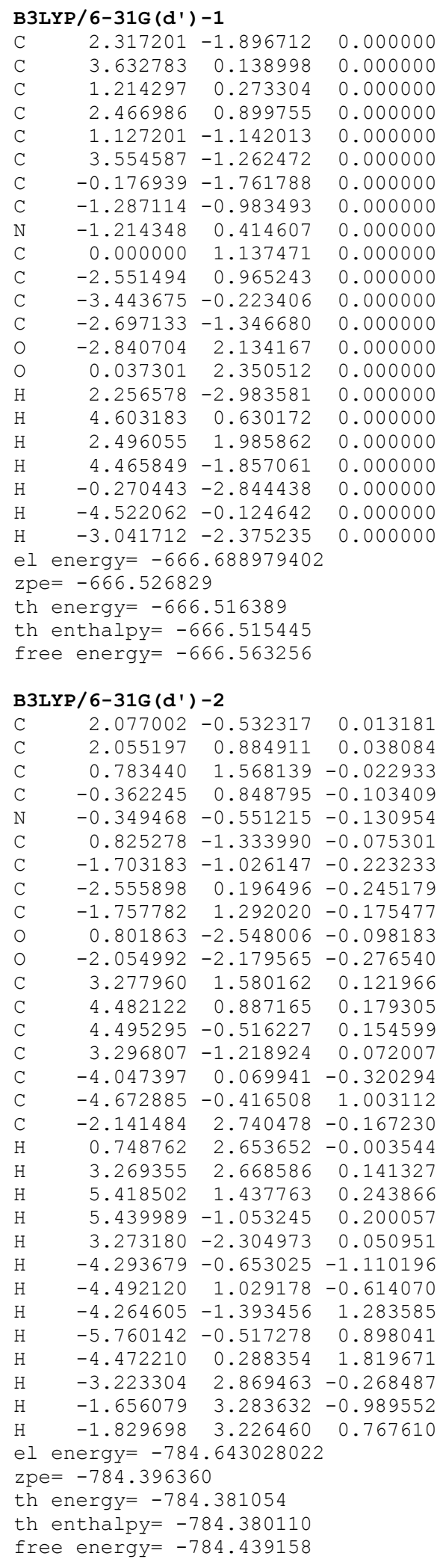

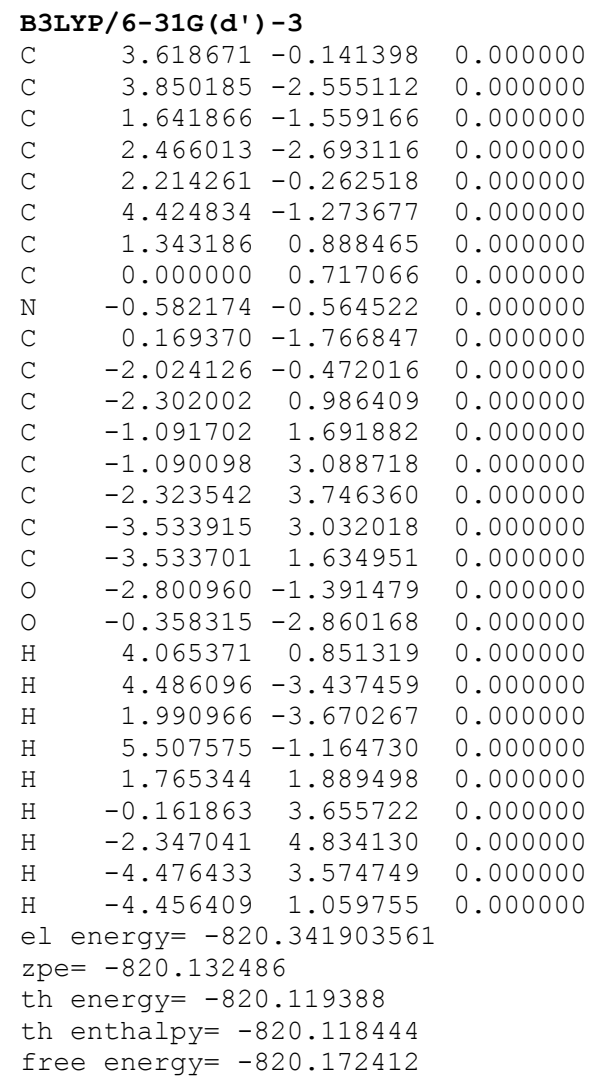

\begin{tabular}{|c|c|c|c|}
\hline \multicolumn{4}{|c|}{ B3LYP/6-31G (d' $)-4$} \\
\hline $\mathrm{C}$ & -2.320220 & 1.409173 & 0.038158 \\
\hline $\mathrm{C}$ & -3.495794 & -0.715318 & 0.064770 \\
\hline & -1.089696 & -0.682804 & -0.039470 \\
\hline c & -2.288828 & -1.407561 & 0.004009 \\
\hline c & -1.071628 & 0.727507 & -0.023038 \\
\hline c & -3.510037 & 0.695978 & 0.081417 \\
\hline c & 0.184486 & 1.429533 & -0.067450 \\
\hline C & 1.340608 & 0.722642 & -0.125974 \\
\hline $\mathrm{N}$ & 1.340402 & -0.678897 & -0.146523 \\
\hline C & 0.176588 & -1.469406 & -0.105001 \\
\hline c & 2.701441 & -1.143094 & -0.215531 \\
\hline C & 3.540974 & 0.084794 & -0.230438 \\
\hline C & 2.730636 & 1.174505 & -0.178196 \\
\hline 0 & 3.061647 & -2.295626 & -0.257178 \\
\hline O & 0.205939 & -2.685062 & -0.121193 \\
\hline C & 5.035175 & -0.026788 & -0.282728 \\
\hline C & 5.647479 & -0.501238 & 1.050892 \\
\hline C & 3.103969 & 2.625882 & -0.170320 \\
\hline O & -2.242786 & 2.768388 & 0.049918 \\
\hline C & -3.449641 & 3.513901 & 0.103215 \\
\hline O & -4.723195 & -1.296448 & 0.111860 \\
\hline C & -4.790290 & -2.716713 & 0.101077 \\
\hline $\mathrm{H}$ & -2.226533 & -2.489179 & -0.011701 \\
\hline $\mathrm{H}$ & -4.475577 & 1.187112 & 0.129344 \\
\hline $\mathrm{H}$ & 0.197919 & 2.513055 & -0.053087 \\
\hline $\mathrm{H}$ & 5.301650 & -0.749702 & -1.066242 \\
\hline $\mathrm{H}$ & 5.474410 & 0.935939 & -0.573921 \\
\hline $\mathrm{H}$ & 5.244441 & -1.480940 & 1.329555 \\
\hline $\mathrm{H}$ & 6.737312 & -0.591873 & 0.962542 \\
\hline $\mathrm{H}$ & 5.427813 & 0.204672 & 1.861730 \\
\hline $\mathrm{H}$ & 4.185885 & 2.762881 & -0.260262 \\
\hline
\end{tabular}




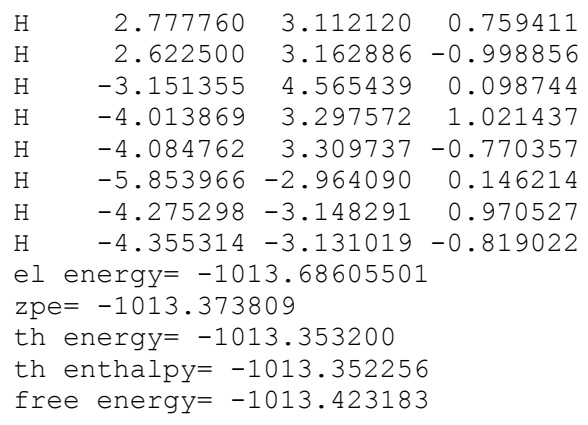

\section{B3LYP / 6-31G (d' ) -5}

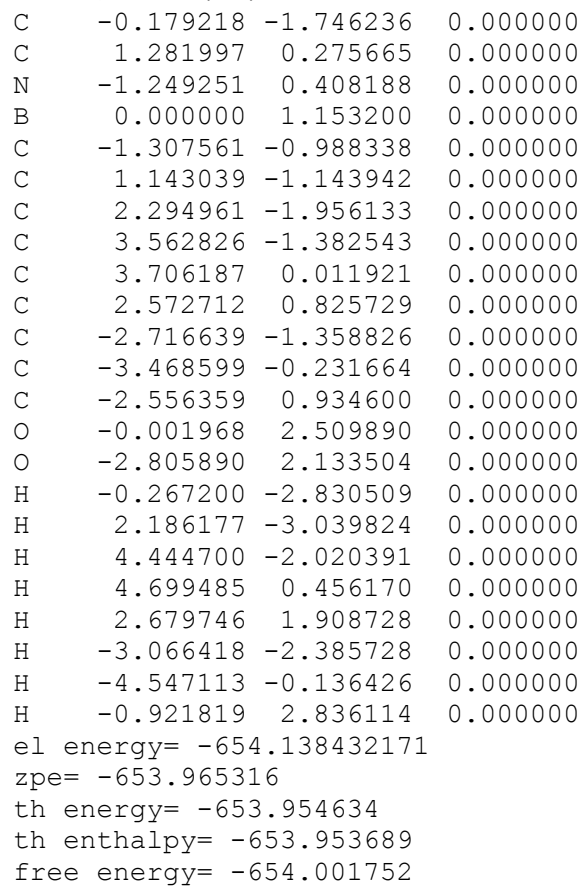

\section{B3LYP/ 6-31G (d' ) -6}

$\begin{array}{lrrr}\text { C } & 0.805707 & 1.543351 & -0.026488 \\ \mathrm{C} & 2.142417 & -0.565587 & 0.015653 \\ \mathrm{~N} & -0.385859 & -0.537239 & -0.129806 \\ \mathrm{~B} & 0.811737 & -1.359807 & -0.075149 \\ \mathrm{C} & -0.365529 & 0.860749 & -0.104494 \\ \mathrm{C} & 2.088123 & 0.859210 & 0.035784 \\ \mathrm{C} & 3.285416 & 1.598229 & 0.116482 \\ \mathrm{C} & 4.514633 & 0.948399 & 0.176686 \\ \mathrm{C} & 4.574516 & -0.452037 & 0.157655 \\ \mathrm{C} & 3.395813 & -1.193958 & 0.077724 \\ \mathrm{C} & -1.755036 & 1.328544 & -0.175355 \\ \mathrm{C} & -2.572533 & 0.239660 & -0.241314 \\ \mathrm{C} & -1.716629 & -0.972566 & -0.214764 \\ \mathrm{O} & 0.722838 & -2.714350 & -0.104115 \\ \mathrm{O} & -2.047491 & -2.153594 & -0.259280 \\ \mathrm{C} & -4.066326 & 0.133764 & -0.315013 \\ \mathrm{C} & -4.695392 & -0.381005 & 0.995597 \\ \mathrm{C} & -2.125872 & 2.780160 & -0.170685 \\ \mathrm{H} & 0.791144 & 2.630663 & -0.008972 \\ \mathrm{H} & 3.242647 & 2.686532 & 0.131491 \\ \mathrm{H} & 5.431612 & 1.531562 & 0.238818 \\ \mathrm{H} & 5.537657 & -0.956200 & 0.205212 \\ \mathrm{H} & 3.436747 & -2.281448 & 0.062365 \\ \mathrm{H} & -0.214460 & -2.979018 & -0.164414 \\ \mathrm{H} & -4.500345 & 1.106102 & -0.580702 \\ \mathrm{H} & -4.327189 & -0.561887 & -1.125115\end{array}$

$\begin{array}{lrrr}\mathrm{H} & -4.484168 & 0.299011 & 1.830361 \\ \mathrm{H} & -5.784203 & -0.466749 & 0.891837 \\ \mathrm{H} & -4.297219 & -1.369589 & 1.249153 \\ \mathrm{H} & -1.808919 & 3.264894 & 0.763029 \\ \mathrm{H} & -3.206201 & 2.921428 & -0.273156 \\ \mathrm{H} & -1.633395 & 3.316476 & -0.993182\end{array}$ el energy $=-772.091720699$ $\mathrm{zpe}=-771.834107$

th energy $=-771.818539$

th enthalpy $=-771.817595$

free energy $=-771.876906$

\section{B3LYP/6-31G (d' ) -7}

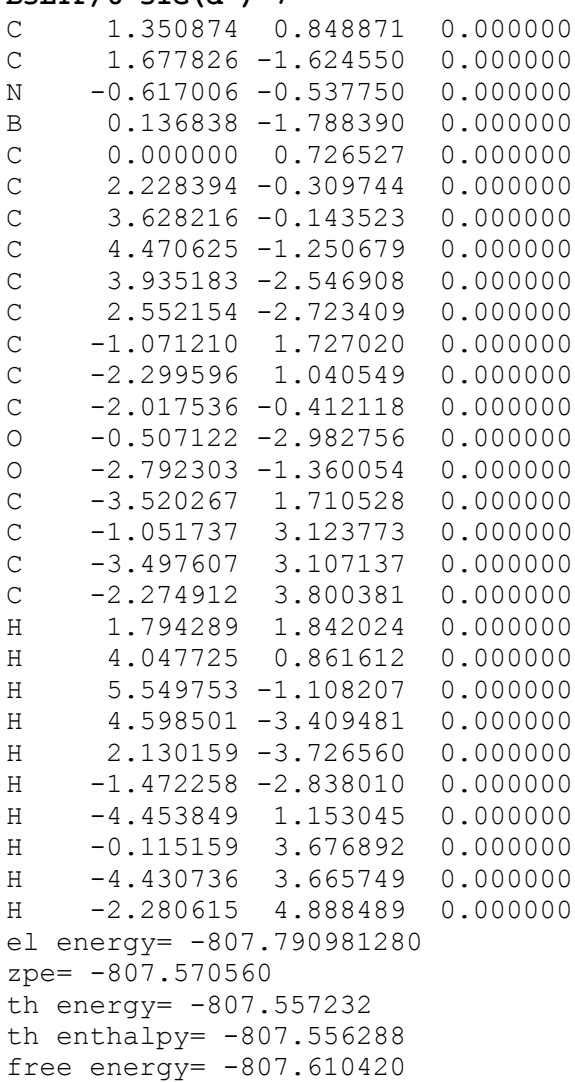

\section{B3LYP / 6-31G (d' ) -8}

$\begin{array}{lll}-2.405388 & -1.525005 & 0.018476\end{array}$

$-2.444147 \quad 1.333710-0.012016$

$-1.194166-0.785634-0.036326$

$\begin{array}{llll}-3.581331 & -0.809906 & 0.057755\end{array}$

$\begin{array}{lll}-3.603541 & 0.589356 & 0.043048\end{array}$

$\begin{array}{lll}-1.212792 & 0.640428 & -0.051537\end{array}$

$\begin{array}{lll}-4.903459 & 1.026566 & 0.074296\end{array}$

$\begin{array}{lll}-5.712109 & -0.142840 & 0.222395\end{array}$

$\begin{array}{lll}-4.862598 & -1.290562 & 0.099955\end{array}$

$0.137667 \quad 1.398316-0.111350$

$\begin{array}{llll}1.316897 & 0.550182-0.144944\end{array}$

$1.263309-0.845399-0.126613$

$0.072087-1.497325-0.075378$

$2.641283-1.345932-0.171570$

$3.486346-0.276259-0.215736$

$2.659397 \quad 0.955241-0.200672$

$\begin{array}{llll}0.255963 & 2.752021 & -0.132298\end{array}$

$3.017467 \quad 2.128953-0.232789$

$4.983511-0.205949-0.259157$

$\begin{array}{llll}5.599354 & 0.283003 & 1.067490\end{array}$

$2.978077-2.805930-0.166983$

$\begin{array}{llll}-2.406961 & -2.611702 & 0.028810\end{array}$ 


\begin{tabular}{|c|c|c|c|}
\hline $\mathrm{H}$ & -2.466971 & 2.419679 & -0.025586 \\
\hline & -6.182668 & -0.142003 & 1.217011 \\
\hline & -6.470059 & -0.166228 & -0.570055 \\
\hline & 0.059622 & -2.584655 & -0.062575 \\
\hline & 1.199799 & 2.996126 & -0.172585 \\
\hline & 5.399470 & -1.186326 & -0.524613 \\
\hline & 5.277256 & 0.489476 & -1.058157 \\
\hline & 6.691806 & 0.343395 & 0.985726 \\
\hline & 5.220017 & 1.278877 & 1.321608 \\
\hline & 5.355581 & -0.398133 & 1.892455 \\
\hline & 2.630526 & -3.288071 & 0.757186 \\
\hline & 4.056776 & -2.971861 & -0.247618 \\
\hline & 2.490863 & -3.326601 & -1.002611 \\
\hline & $r g y=-960$ & .618216265 & \\
\hline & 960.34504 & & \\
\hline & $r g y=-9$ & .32706 & \\
\hline & halpy= & 326124 & \\
\hline & energy $=-9$ & 360.391600 & \\
\hline & $/ 6-31 G\left(d^{\prime}\right)$ & -9 & \\
\hline & -4.783440 & -1.619718 & -0.022535 \\
\hline & -5.754402 & -0.568031 & 0.054859 \\
\hline & -5.064366 & 0.681467 & -0.021872 \\
\hline & -3.725926 & 0.381336 & -0.008266 \\
\hline & -3.559079 & -1.008771 & -0.009440 \\
\hline & -2.315172 & -1.598553 & -0.009322 \\
\hline & -1.185732 & -0.737433 & -0.006734 \\
\hline & -1.351322 & 0.678637 & -0.005540 \\
\hline & -2.649235 & 1.241451 & -0.007132 \\
\hline$B$ & -0.089861 & 1.573715 & -0.002036 \\
\hline J & 1.181873 & 0.857313 & -0.000618 \\
\hline 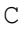 & 1.266530 & -0.545437 & -0.001701 \\
\hline & 0.147158 & -1.313069 & -0.004390 \\
\hline 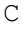 & 2.689882 & -0.890995 & 0.000458 \\
\hline C & 3.422718 & 0.311031 & 0.002738 \\
\hline z & 2.462930 & 1.437207 & 0.002075 \\
\hline 0 & 2.670817 & 2.643936 & 0.003490 \\
\hline o & -0.117900 & 2.931590 & -0.000608 \\
\hline C & 4.815030 & 0.327385 & 0.005126 \\
\hline C & 5.482277 & -0.899805 & 0.005246 \\
\hline 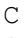 & 4.757999 & -2.104471 & 0.003019 \\
\hline C & 3.360239 & -2.116813 & 0.000595 \\
\hline $\mathrm{H}$ & -6.288649 & -0.635716 & 1.013892 \\
\hline I & -6.450685 & -0.654337 & -0.788701 \\
\hline $\mathrm{H}$ & -2.204684 & -2.679626 & -0.011645 \\
\hline & -2.783397 & 2.319385 & -0.008180 \\
\hline & 0.247933 & -2.395946 & -0.00522 \\
\hline
\end{tabular}

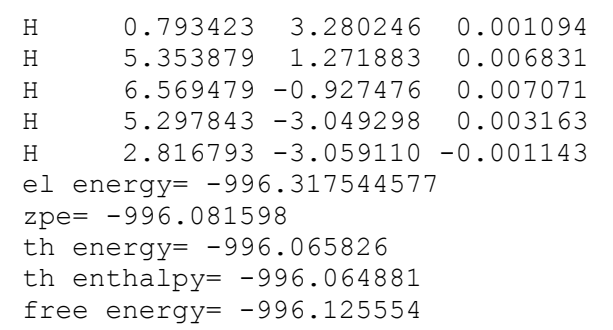

\section{B3LYP / 6-31G (d' ) -10}

\begin{tabular}{|c|c|c|c|}
\hline $\mathrm{C}$ & -3.261088 & -1.432148 & 0.111417 \\
\hline S & -4.506904 & -0.232370 & 0.163460 \\
\hline C & -3.349270 & 1.056030 & 0.076117 \\
\hline C & -2.059407 & 0.594235 & 0.009476 \\
\hline C & -2.009112 & -0.858080 & 0.03026 \\
\hline C & -0.741368 & -1.558103 & -0.03035 \\
\hline C & 0.424144 & -0.864371 & -0.10724 \\
\hline N & 0.449636 & 0.540804 & -0.13277 \\
\hline B & -0.733728 & 1.390557 & -0.0802 \\
\hline $\mathrm{C}$ & 1.781260 & 0.967885 & -0.21 \\
\hline C & 2.632038 & -0.246152 & -0.24 \\
\hline $\mathrm{C}$ & 1.811006 & $-1 \cdot 33$ & -0.1 \\
\hline 0 & 2.120182 & 2.148324 & -0.25 \\
\hline O & -0.621062 & 2.74 & -0.1 \\
\hline C & 4.126238 & -0.145333 & -0.31 \\
\hline $\mathrm{C}$ & 4.754832 & 0.36 & 1.0 \\
\hline $\mathrm{C}$ & 2.180277 & -2.785596 & -0.17 \\
\hline $\mathrm{H}$ & -3.515964 & -2.484 & 0.1 \\
\hline $\mathrm{H}$ & -3.687203 & 2.085751 & 0.07 \\
\hline $\mathrm{H}$ & -0.722608 & -2.644 & -0.01 \\
\hline $\mathrm{H}$ & 0.323431 & 2.98 & -0.1 \\
\hline $\mathrm{H}$ & 4.5576 & $-1 \cdot 1$ & -0.5 \\
\hline $\mathrm{H}$ & 4.390307 & 0.55 & $-1 \cdot 11$ \\
\hline $\mathrm{H}$ & 4.5 & -0.3212 & 1.8 \\
\hline $\mathrm{H}$ & 5.844093 & 0.44 & 0.900 \\
\hline $\mathrm{H}$ & 4.359206 & 1.35 & 1.2 \\
\hline $\mathrm{H}$ & 3.260588 & -2.927 & -0.2 \\
\hline $\mathrm{H}$ & 1.688713 & -3.321888 & -0.9 \\
\hline $\mathrm{H}$ & 1.863086 & -3.270294 & 0.7 \\
\hline \multicolumn{4}{|c|}{$\begin{array}{l}\text { el energy }=-1092.84753491 \\
\text { zpe }=-1092.623473\end{array}$} \\
\hline \multicolumn{4}{|c|}{$\mathrm{zpe}=-1092.623473$} \\
\hline \multicolumn{4}{|c|}{ th energy $=-1092.608241$} \\
\hline \multicolumn{4}{|c|}{ th enthalpy $=-1092.607297$} \\
\hline & energ & 092 & \\
\hline
\end{tabular}




\section{TD-B3LYP/6-31G $\left(d^{\prime}\right)$ coordinates for compounds 1,3-10}

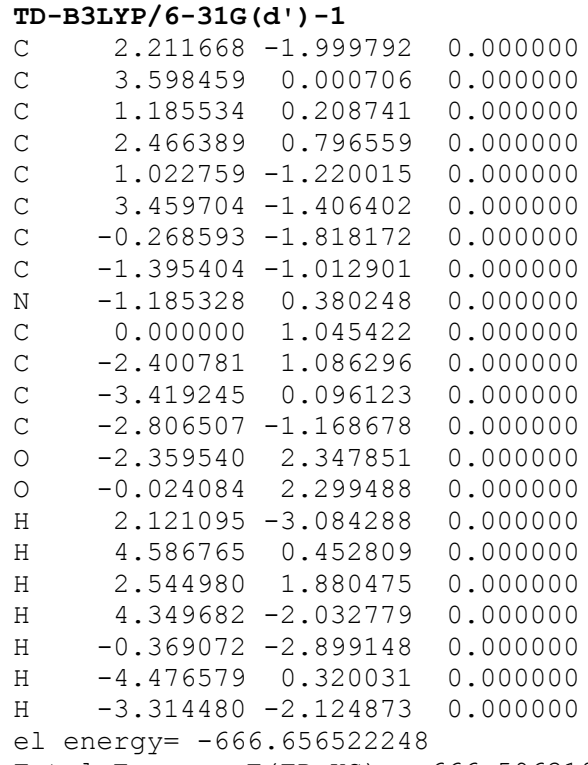

Total Energy, E (TD-KS $)=-666.596319616$

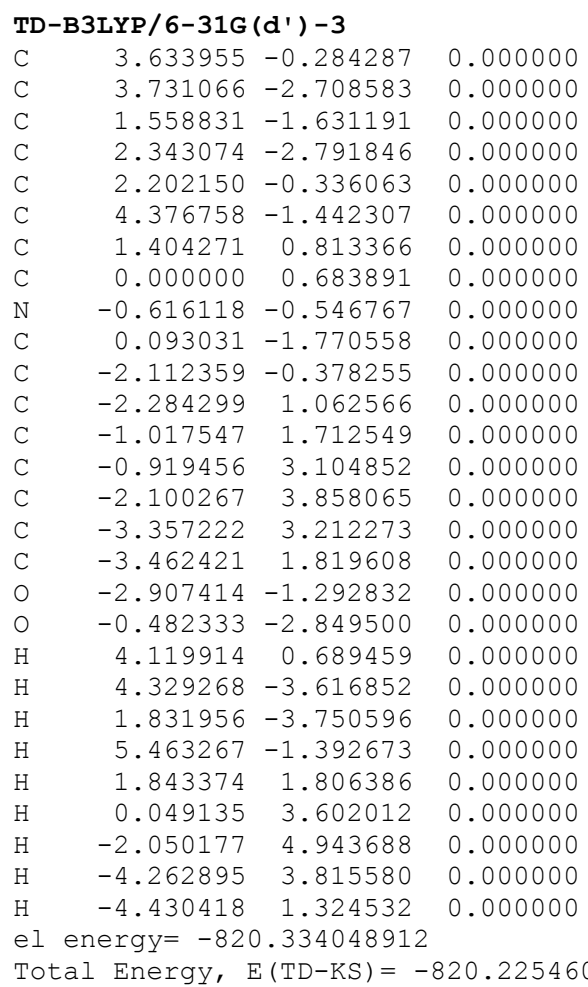

TD-B3LYP / 6-31G (d' ) -4

$\begin{array}{lrrr}\text { C } & -2.340154 & 1.414769 & 0.038718 \\ \text { C } & -3.484635 & -0.718308 & 0.066630 \\ \text { C } & -1.063742 & -0.705329 & -0.040599 \\ \text { C } & -2.272905 & -1.411838 & 0.004672 \\ \text { C } & -1.062448 & 0.735450 & -0.025358 \\ \text { C } & -3.520526 & 0.704779 & 0.083516 \\ \text { C } & 0.155137 & 1.430139 & -0.072390 \\ \text { C } & 1.347656 & 0.701738 & -0.132042 \\ \text { N } & 1.355625 & -0.697887 & -0.150268\end{array}$

C $\quad 0.180961-1.484338-0.105927$

$2.721836-1.153805-0.217760$

$3.5326020 .076954-0.237717$

$2.702312 \quad 1.185097-0.182993$

$3.100095-2.313845-0.250352$

$0.207272-2.709329-0.121936$

$5.022611-0.006880-0.284010$

$5.635437-0.440357 \quad 1.067857$

$3.061100 \quad 2.639836-0.177370$

$\begin{array}{lll}-2.251812 & 2.764000 & 0.047944\end{array}$

$\begin{array}{lll}-3.453368 & 3.523849 & 0.102327\end{array}$

$\begin{array}{lll}-4.701017 & -1.302741 & 0.115875\end{array}$

$\begin{array}{llll}-4.778399 & -2.726733 & 0.104930\end{array}$

$-2.215395-2.494434-0.009787$

$\begin{array}{lll}-4.490964 & 1.184544 & 0.132482\end{array}$

$0.184312 \quad 2.512554-0.059190$

$5.304056-0.753548-1.040157$

$5.451795 \quad 0.954140-0.596650$

$5.243750-1.420134 \quad 1.360943$

$\begin{array}{llll}6.727492 & -0.513678 & 0.985948\end{array}$

$\begin{array}{llll}5.397876 & 0.280195 & 1.860428\end{array}$

$\begin{array}{llll}4.143277 & 2.780646 & -0.266721\end{array}$

$\begin{array}{lll}2.739299 & 3.137387 & 0.750099\end{array}$

$2.586413 \quad 3.181335-1.009270$

$\begin{array}{llll}-3.143705 & 4.571456 & 0.096028\end{array}$

$\begin{array}{lll}-4.014128 & 3.308424 & 1.022019\end{array}$

$\begin{array}{llll}-4.087839 & 3.319107 & -0.770677\end{array}$

$\begin{array}{llll}-5.843861 & -2.963555 & 0.151306\end{array}$

$\begin{array}{llll}-4.263469 & -3.156127 & 0.974305\end{array}$

$-4.345440-3.137918-0.816387$

el energy $=-1013.68074386$

Total Energy, $\mathrm{E}(\mathrm{TD}-\mathrm{KS})=-1013.57871932$

TD-B3LYP/ 6-31G (d' ) -5

$\begin{array}{lll}-0.155103 & -1.763052 & 0.000000\end{array}$

$\begin{array}{lll}1.273036 & 0.278054 & 0.000000\end{array}$

$\begin{array}{lll}-1.249538 & 0.409779 & 0.000000\end{array}$

$0.000000 \quad 1.151808 \quad 0.000000$

$-1.321609-0.9736430 .000000$

$1.129244-1.1739370 .000000$

$2.310671-1.9888170 .000000$

$3.559774-1.4127320 .000000$

$\begin{array}{lll}3.697443 & 0.002663 & 0.000000\end{array}$

$\begin{array}{llll}2.566189 & 0.817959 & 0.000000\end{array}$

$-2.719811-1.353790 \quad 0.000000$

$\begin{array}{lll}-3.470548 & -0.192707 & 0.000000\end{array}$

$\begin{array}{lll}-2.569712 & 0.952809 & 0.000000\end{array}$

$\begin{array}{lll}-0.000705 & 2.512165 & 0.000000\end{array}$

$\begin{array}{lll}-2.781237 & 2.172406 & 0.000000\end{array}$

$-0.261195-2.8448550 .000000$

$2.200263-3.0718830 .000000$

$4.449491-2.0389630 .000000$

$\begin{array}{lll}4.691501 & 0.445086 & 0.000000\end{array}$

$2.677517 \quad 1.900506 \quad 0.000000$

$\begin{array}{lll}-3.072514 & -2.377637 & 0.000000\end{array}$

$\begin{array}{lll}-4.549344 & -0.096664 & 0.000000\end{array}$

$\begin{array}{lll}-0.930871 & 2.823505 & 0.000000\end{array}$

el energy $=-654.132966859$

Total Energy, $\mathrm{E}(\mathrm{TD}-\mathrm{KS})=-654.019267108$

\begin{tabular}{lrrr}
\multicolumn{4}{l}{ TD-B3LYP/6-31G( $\left.\mathbf{d}^{\prime}\right) \mathbf{~} \mathbf{6}$} \\
C & 0.840072 & 1.554985 & -0.029851 \\
C & 2.121244 & -0.580415 & 0.016961 \\
N & -0.393959 & -0.546048 & -0.132015 \\
B & 0.798795 & -1.368414 & -0.072837 \\
C & -0.376732 & 0.846672 & -0.110884 \\
C & 2.076721 & 0.879924 & 0.033981 \\
C & 3.308640 & 1.611952 & 0.116505 \\
C & 4.513578 & 0.953114 & 0.178268
\end{tabular}




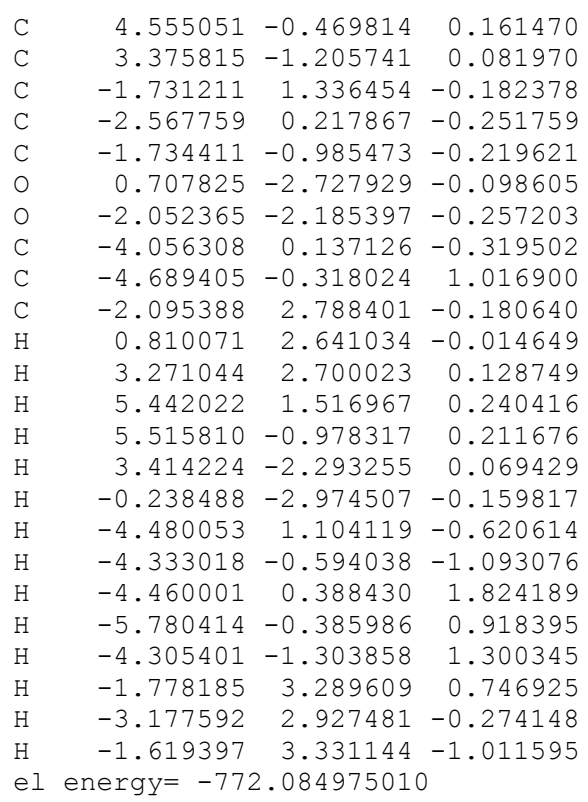

Total Energy, E (TD-KS $)=-771.973468370$

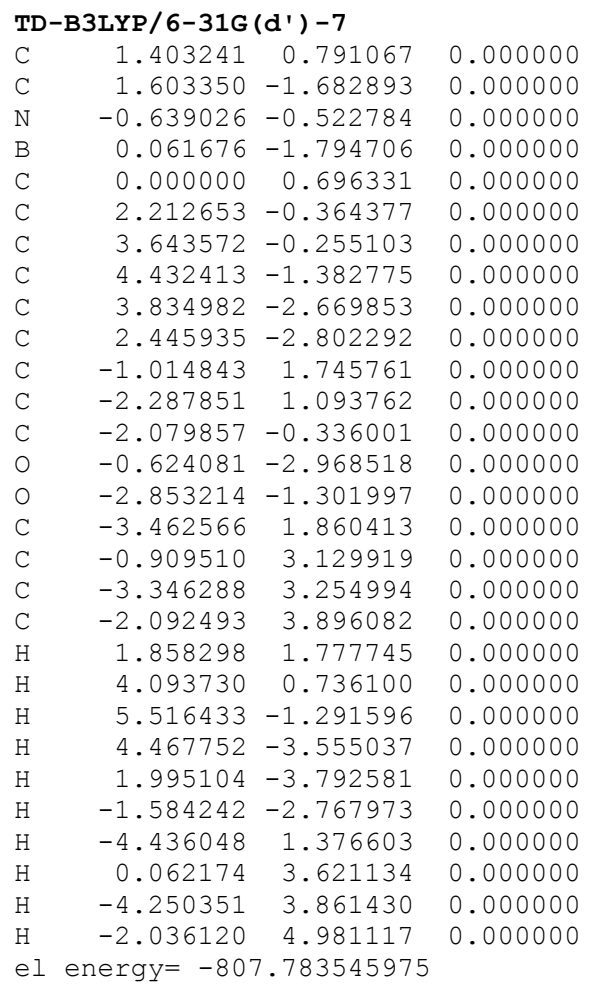

Total Energy, $\mathrm{E}(\mathrm{TD}-\mathrm{KS})=-807.676690163$

$\begin{array}{lrrr}\left.\text { TD-B3LYP/6-31G( } \mathbf{d}^{\prime}\right) \mathbf{~} \mathbf{8} & \\ \text { C } & 2.412475 & -1.551219 & -0.065367 \\ \text { C } & 2.420746 & 1.342968 & -0.018943 \\ \text { C } & 1.190033 & -0.834065 & 0.003415 \\ \text { C } & 3.571985 & -0.811549 & -0.108007 \\ \text { C } & 3.584887 & 0.611592 & -0.085620 \\ \text { C } & 1.203691 & 0.634297 & 0.026738 \\ \text { O } & 4.877190 & 1.043853 & -0.140161 \\ \text { C } & 5.698039 & -0.115366 & -0.199091 \\ \text { O } & 4.847399 & -1.264957 & -0.175942 \\ \text { B } & -0.146193 & 1.386647 & 0.102766\end{array}$

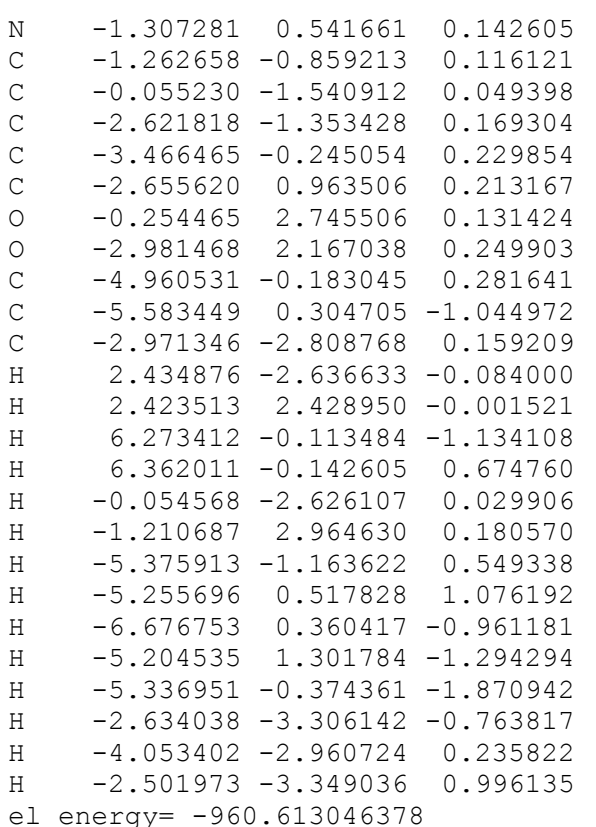

rgy $=-960.613046378$

Total Energy, E (TD-KS $)=-960.513660531$

\section{TD-B3LYP / 6-31G (d') -9}

$\begin{array}{lllll}0 & -4.776418 & -1.605661 & 0.000106\end{array}$ $-5.738592-0.550735-0.000287$

$\begin{array}{lll}-5.033200 & 0.690002 & -0.000088\end{array}$

$\begin{array}{llll}-3.708465 & 0.391325 & -0.000075\end{array}$

$\begin{array}{lll}-3.548940 & -1.022985 & 0.000052\end{array}$

$\begin{array}{lll}-2.320299 & -1.629080 & 0.000154\end{array}$

$\begin{array}{lll}-1.177009 & -0.777254 & 0.000139\end{array}$

$\begin{array}{lll}-1.342041 & 0.677392 & 0.000001\end{array}$

$-2.626694 \quad 1.248395-0.000100$

$\begin{array}{lll}-0.076076 & 1.570951-0.000080\end{array}$

$\begin{array}{lll}1.172166 & 0.854067 & 0.000143\end{array}$

$\begin{array}{lll}1.260369 & -0.532381 & 0.000252\end{array}$

$0.132716-1.340148 \quad 0.000221$

$\begin{array}{lll}2.680928 & -0.881189 & 0.000165\end{array}$

$\begin{array}{lll}3.415497 & 0.345381 & 0.000095\end{array}$

$\begin{array}{lll}2.486800 & 1.450201 & 0.000491\end{array}$

$\begin{array}{lll}2.638248 & 2.683981 & 0.000145\end{array}$

$-0.116712 \quad 2.931326-0.000176$

$\begin{array}{llll}4.820140 & 0.317604 & -0.000230\end{array}$

$5.459666-0.922497-0.000380$

$4.733670-2.132292-0.000245$

$3.328019-2.109930 \quad 0.000016$

$\begin{array}{lll}-6.354619 & -0.618754 & 0.905355\end{array}$

$\begin{array}{llll}-6.353978 & -0.618840 & -0.906372\end{array}$

$\begin{array}{lll}-2.220220 & -2.710175 & 0.000232\end{array}$

$\begin{array}{llll}-2.750781 & 2.327331 & -0.000208\end{array}$

$\begin{array}{llll}0.253262 & -2.419128 & 0.000250\end{array}$

$\begin{array}{llll}0.810931 & 3.256206 & -0.000231\end{array}$

$\begin{array}{llll}5.386700 & 1.245480 & -0.000258\end{array}$

$6.547869-0.959393-0.000565$

$5.261675-3.082211-0.000374$

$2.764428-3.041758 \quad 0.000053$

el energy $=-996.311510519$

Total Energy, $E(T D-K S)=-996.214884711$

\section{TD-B3LYP/ 6-31G (d' ) -10}

$\begin{array}{lll}-3.285455 & -1.449298 & 0.104743\end{array}$

$\begin{array}{llll}-4.511327 & -0.265469 & 0.169401\end{array}$

$\begin{array}{lll}-3.348666 & 1.042350 & 0.090176\end{array}$

$\begin{array}{llll}-2.058343 & 0.588905 & 0.016575\end{array}$

$\begin{array}{lll}-1.995342 & -0.871773 & 0.023407\end{array}$

$-0.756631-1.563619-0.045445$ 


$\begin{array}{rrr}0.442687 & -0.831377 & -0.121715 \\ 0.441520 & 0.553981 & -0.131883 \\ -0.739280 & 1.395353 & -0.069093 \\ 1.803195 & 1.009685 & -0.218977 \\ 2.642958 & -0.172759 & -0.261760 \\ 1.820201 & -1.299491 & -0.199412 \\ 2.082378 & 2.216714 & -0.248748 \\ -0.641866 & 2.747794 & -0.087103 \\ 4.134798 & -0.080834 & -0.328331 \\ 4.788547 & 0.167855 & 1.049546 \\ 2.186156 & -2.746988 & -0.207612 \\ -3.533686 & -2.503961 & 0.126983 \\ -3.697988 & 2.068229 & 0.103473\end{array}$

$\mathrm{H} \quad-0.719624-2.648146-0.037971$

$\mathrm{H} \quad 0.308171 \quad 2.986602-0.147613$

$\mathrm{H} \quad 4.550049-0.996973-0.770588$

$\begin{array}{lllll}\mathrm{H} & 4.406863 & 0.748849 & -0.995577\end{array}$

$\mathrm{H} \quad 4.559094 \quad-0.645283 \quad 1.749684$

$\begin{array}{llll}\mathrm{H} & 5.879905 & 0.239574 & 0.952707\end{array}$

$\mathrm{H} \quad 4.421187 \quad 1.104952 \quad 1.483508$

$\mathrm{H} \quad 3.269950 \quad-2.885320 \quad-0.285543$

$\mathrm{H} \quad 1.721424-3.285609-1.050284$

$\begin{array}{lllll}\mathrm{H} & 1.852918 & -3.262047 & 0.709076\end{array}$

el energy $=-1092.84146834$

Total Energy, E (TD-KS $)=-1092.72395857$ 


\section{Thermal Ellipsoid Plot for Compound 4 and Compound 6}

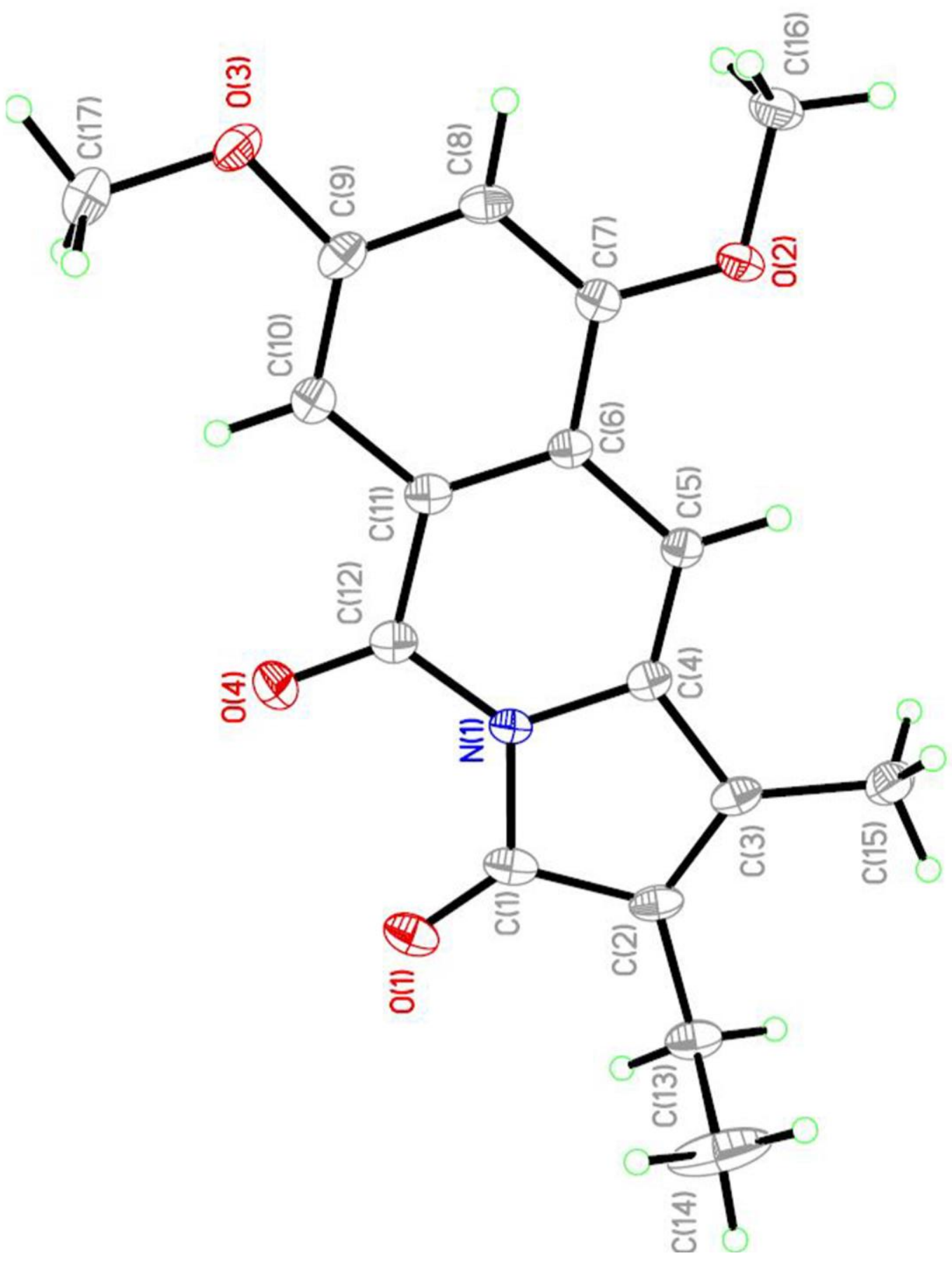

Molecular structure of $\mathbf{4}$ from X-ray diffraction. ORTEP of single molecule with thermal ellipsoids drawn at 50\% probability. (gray: carbon, red: oxygen; blue: nitrogen; green: hydrogen. 


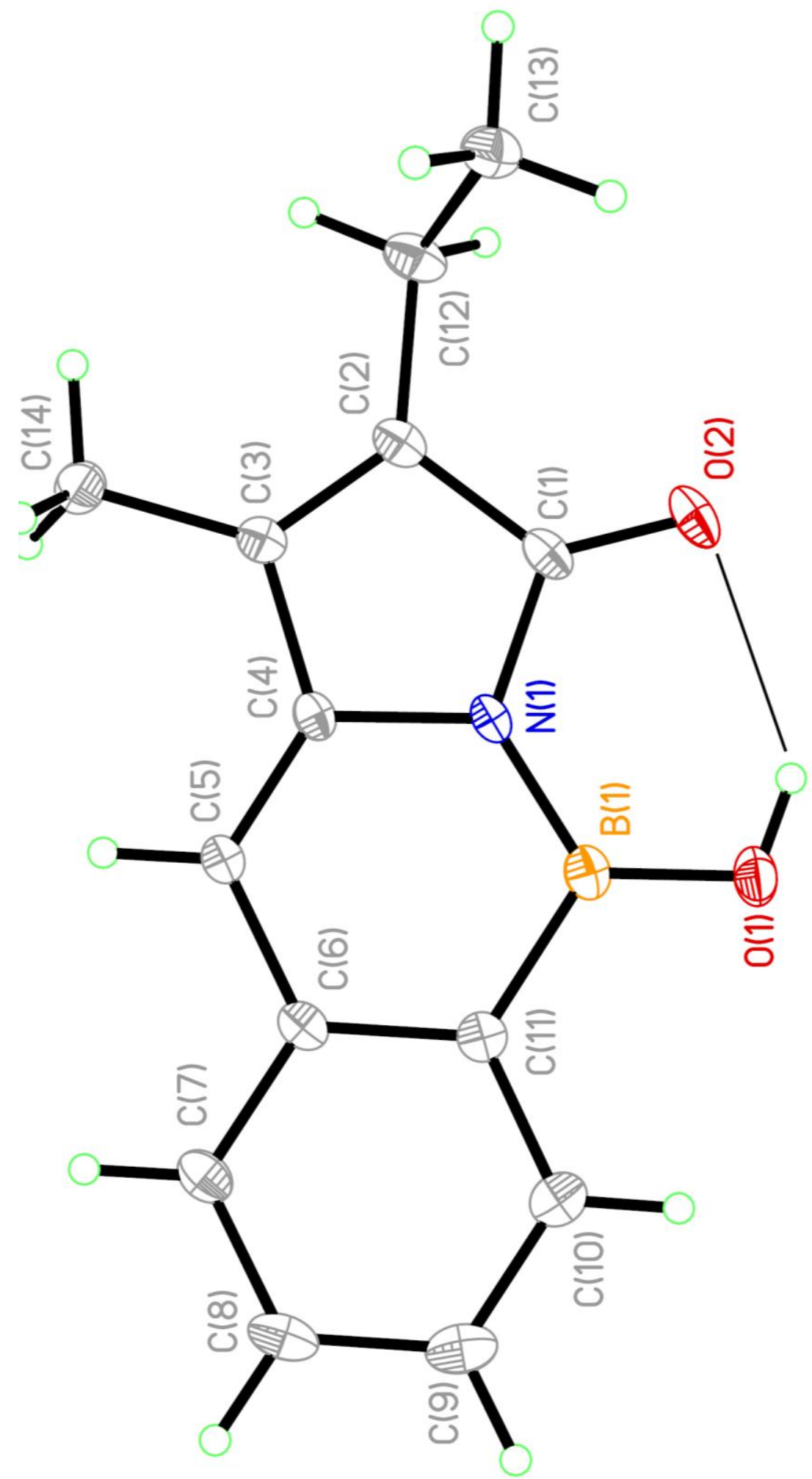

Molecular structure of $\mathbf{6}$ from X-ray diffraction. ORTEP of single molecule with thermal ellipsoids drawn at 50\% probability. (gray: carbon, red: oxygen; blue: nitrogen; green: hydrogen; orange: boron) 


\section{X-RAY CRYSTAL DATA}

Table S4. Crystal data and structure refinement for compound 4. CCDC: 1418451

Empirical formula

Formula weight

Temperature

Wavelength

Crystal system

Space group

Unit cell dimensions

Volume

Z

Density (calculated)

Absorption coefficient

$\mathrm{F}(000)$

Crystal size

Theta range for data collection

Index ranges

Reflections collected

Independent reflections

Completeness to theta $=25.242^{\circ}$

Absorption correction

Refinement method

Data / restraints / parameters

Goodness-of-fit on $\mathrm{F}^{2}$

Final $R$ indices [ $>2$ sigma(I)]

$R$ indices (all data)

Extinction coefficient

Largest diff. peak and hole
$\mathrm{C}_{17} \mathrm{H}_{17} \mathrm{~N} \mathrm{O}_{4}$

299.32

$100(2) \mathrm{K}$

$0.71073 \AA$

Orthorhombic

P c c n

$\mathrm{a}=16.6112(11) \AA$

$\alpha=90^{\circ}$

$\mathrm{b}=24.0764(16) \AA$

$\beta=90^{\circ}$

$\mathrm{c}=7.0730(5) \AA$

$\gamma=90^{\circ}$

2828.8(3) $\AA^{3}$

8

$1.406 \mathrm{Mg} / \mathrm{m}^{3}$

$0.101 \mathrm{~mm}^{-1}$

1264

$0.540 \times 0.047 \times 0.024 \mathrm{~mm}^{3}$

1.489 to $24.997^{\circ}$.

$-19<=\mathrm{h}<=19,-28<=\mathrm{k}<=28,-8<=\mathrm{k}<=8$

37772

$2499[\mathrm{R}$ (int) $=0.0731]$

$100 \%$

Semi-empirical from equivalents

Full-matrix least-squares on $\mathrm{F}^{2}$

2499 / 0 / 203

1.017

$\mathrm{R} 1=0.0440, w R 2=0.1034$

$\mathrm{R} 1=0.0637, \mathrm{wR} 2=0.1108$

$\mathrm{n} / \mathrm{a}$

0.328 and -0.248 e. $\AA^{-3}$ 
Table S5. Bond lengths $(\AA)$ and angles $\left({ }^{\circ}\right)$ for compound 4 (Symmetry transformations used to generate equivalent atoms).

\begin{tabular}{|c|c|c|c|}
\hline \multicolumn{2}{|c|}{ Bond lengths ( $\AA$ ) } & $C(17)-H(17 C)$ & 0.9800 \\
\hline$N(1)-C(12)$ & $1.404(2)$ & & \\
\hline$N(1)-C(4)$ & $1.405(2)$ & & \\
\hline$N(1)-C(1)$ & $1.434(2)$ & \multicolumn{2}{|c|}{ Bond angles $\left({ }^{\circ}\right)$} \\
\hline$C(1)-O(1)$ & $1.209(2)$ & $C(12)-N(1)-C(4)$ & 123.96(15) \\
\hline$C(1)-C(2)$ & $1.479(3)$ & $C(12)-N(1)-C(1)$ & $127.35(15)$ \\
\hline$C(2)-C(3)$ & $1.342(3)$ & $C(4)-N(1)-C(1)$ & 108.67(15) \\
\hline$C(2)-C(13)$ & $1.500(3)$ & $\mathrm{O}(1)-\mathrm{C}(1)-\mathrm{N}(1)$ & 125.59(17) \\
\hline$C(3)-C(4)$ & $1.457(3)$ & $O(1)-C(1)-C(2)$ & $128.87(17)$ \\
\hline$C(3)-C(15)$ & $1.490(3)$ & $N(1)-C(1)-C(2)$ & 105.54(15) \\
\hline$C(4)-C(5)$ & $1.339(3)$ & $C(3)-C(2)-C(1)$ & $109.20(16)$ \\
\hline$C(5)-C(6)$ & $1.440(3)$ & $C(3)-C(2)-C(13)$ & 130.16(18) \\
\hline$C(5)-H(5 A)$ & 0.9500 & $C(1)-C(2)-C(13)$ & 120.64(17) \\
\hline$C(6)-C(11)$ & $1.401(3)$ & $C(2)-C(3)-C(4)$ & 109.14(17) \\
\hline$C(6)-C(7)$ & $1.414(3)$ & $C(2)-C(3)-C(15)$ & 129.77(18) \\
\hline$C(7)-O(2)$ & $1.362(2)$ & $C(4)-C(3)-C(15)$ & 121.09(17) \\
\hline$C(7)-C(8)$ & $1.374(3)$ & $C(5)-C(4)-N(1)$ & $121.65(17)$ \\
\hline$C(8)-C(9)$ & $1.401(3)$ & $C(5)-C(4)-C(3)$ & 130.91(18) \\
\hline $\mathrm{C}(8)-\mathrm{H}(8 \mathrm{~A})$ & 0.9500 & $N(1)-C(4)-C(3)$ & $107.44(15)$ \\
\hline$C(9)-O(3)$ & $1.370(2)$ & $C(4)-C(5)-C(6)$ & 119.27(17) \\
\hline$C(9)-C(10)$ & $1.378(3)$ & $C(4)-C(5)-H(5 A)$ & 120.4 \\
\hline$C(10)-C(11)$ & $1.401(3)$ & $C(6)-C(5)-H(5 A)$ & 120.4 \\
\hline$C(10)-H(10 A)$ & 0.9500 & $C(11)-C(6)-C(7)$ & $118.20(17)$ \\
\hline$C(11)-C(12)$ & $1.487(3)$ & $C(11)-C(6)-C(5)$ & $120.08(17)$ \\
\hline$C(12)-O(4)$ & $1.216(2)$ & $C(7)-C(6)-C(5)$ & $121.72(17)$ \\
\hline$C(13)-C(14)$ & $1.483(3)$ & $\mathrm{O}(2)-\mathrm{C}(7)-\mathrm{C}(8)$ & $124.47(17)$ \\
\hline$C(13)-H(13 A)$ & 0.9900 & $\mathrm{O}(2)-\mathrm{C}(7)-\mathrm{C}(6)$ & 115.13(16) \\
\hline$C(13)-H(13 B)$ & 0.9900 & $C(8)-C(7)-C(6)$ & $120.40(17)$ \\
\hline$C(14)-H(14 A)$ & 0.9800 & $C(7)-C(8)-C(9)$ & 119.93(17) \\
\hline$C(14)-H(14 B)$ & 0.9800 & $\mathrm{C}(7)-\mathrm{C}(8)-\mathrm{H}(8 \mathrm{~A})$ & 120.0 \\
\hline$C(14)-H(14 C)$ & 0.9800 & $\mathrm{C}(9)-\mathrm{C}(8)-\mathrm{H}(8 \mathrm{~A})$ & 120.0 \\
\hline$C(15)-H(15 A)$ & 0.9800 & $\mathrm{O}(3)-\mathrm{C}(9)-\mathrm{C}(10)$ & $124.33(17)$ \\
\hline$C(15)-H(15 B)$ & 0.9800 & $\mathrm{O}(3)-\mathrm{C}(9)-\mathrm{C}(8)$ & 114.13(17) \\
\hline$C(15)-H(15 C)$ & 0.9800 & $C(10)-C(9)-C(8)$ & $121.54(18)$ \\
\hline$C(16)-O(2)$ & $1.430(2)$ & $C(9)-C(10)-C(11)$ & 118.11(18) \\
\hline$C(16)-H(16 A)$ & 0.9800 & $C(9)-C(10)-H(10 A)$ & 120.9 \\
\hline$C(16)-H(16 B)$ & 0.9800 & $C(11)-C(10)-H(10 A)$ & 120.9 \\
\hline$C(16)-H(16 C)$ & 0.9800 & $C(6)-C(11)-C(10)$ & 121.79(17) \\
\hline$C(17)-O(3)$ & $1.435(2)$ & $C(6)-C(11)-C(12)$ & $121.17(17)$ \\
\hline$C(17)-H(17 A)$ & 0.9800 & $C(10)-C(11)-C(12)$ & 117.04(17) \\
\hline$C(17)-H(17 B)$ & 0.9800 & $\mathrm{O}(4)-\mathrm{C}(12)-\mathrm{N}(1)$ & $122.16(17)$ \\
\hline
\end{tabular}




$\begin{array}{llll}\mathrm{O}(4)-\mathrm{C}(12)-\mathrm{C}(11) & 124.00(18) & \mathrm{C}(3)-\mathrm{C}(15)-\mathrm{H}(15 \mathrm{C}) & 109.5 \\ \mathrm{~N}(1)-\mathrm{C}(12)-\mathrm{C}(11) & 113.84(16) & \mathrm{H}(15 \mathrm{~A})-\mathrm{C}(15)-\mathrm{H}(15 \mathrm{C}) & 109.5 \\ \mathrm{C}(14)-\mathrm{C}(13)-\mathrm{C}(2) & 113.97(18) & \mathrm{H}(15 \mathrm{~B})-\mathrm{C}(15)-\mathrm{H}(15 \mathrm{C}) & 109.5 \\ \mathrm{C}(14)-\mathrm{C}(13)-\mathrm{H}(13 \mathrm{~A}) & 108.8 & \mathrm{O}(2)-\mathrm{C}(16)-\mathrm{H}(16 \mathrm{~A}) & 109.5 \\ \mathrm{C}(2)-\mathrm{C}(13)-\mathrm{H}(13 \mathrm{~A}) & 108.8 & \mathrm{O}(2)-\mathrm{C}(16)-\mathrm{H}(16 \mathrm{~B}) & 109.5 \\ \mathrm{C}(14)-\mathrm{C}(13)-\mathrm{H}(13 \mathrm{~B}) & 108.8 & \mathrm{H}(16 \mathrm{~A})-\mathrm{C}(16)-\mathrm{H}(16 \mathrm{~B}) & 109.5 \\ \mathrm{C}(2)-\mathrm{C}(13)-\mathrm{H}(13 \mathrm{~B}) & 108.8 & \mathrm{O}(2)-\mathrm{C}(16)-\mathrm{H}(16 \mathrm{C}) & 109.5 \\ \mathrm{H}(13 \mathrm{~A})-\mathrm{C}(13)-\mathrm{H}(13 \mathrm{~B}) & 107.7 & \mathrm{H}(16 \mathrm{~A})-\mathrm{C}(16)-\mathrm{H}(16 \mathrm{C}) & 109.5 \\ \mathrm{C}(13)-\mathrm{C}(14)-\mathrm{H}(14 \mathrm{~A}) & 109.5 & \mathrm{H}(16 \mathrm{~B})-\mathrm{C}(16)-\mathrm{H}(16 \mathrm{C}) & 109.5 \\ \mathrm{C}(13)-\mathrm{C}(14)-\mathrm{H}(14 \mathrm{~B}) & 109.5 & \mathrm{O}(3)-\mathrm{C}(17)-\mathrm{H}(17 \mathrm{~A}) & 109.5 \\ \mathrm{H}(14 \mathrm{~A})-\mathrm{C}(14)-\mathrm{H}(14 \mathrm{~B}) & 109.5 & \mathrm{O}(3)-\mathrm{C}(17)-\mathrm{H}(17 \mathrm{~B}) & 109.5 \\ \mathrm{C}(13)-\mathrm{C}(14)-\mathrm{H}(14 \mathrm{C}) & 109.5 & \mathrm{H}(17 \mathrm{~A})-\mathrm{C}(17)-\mathrm{H}(17 \mathrm{~B}) & 109.5 \\ \mathrm{H}(14 \mathrm{~A})-\mathrm{C}(14)-\mathrm{H}(14 \mathrm{C}) & 109.5 & \mathrm{O}(3)-\mathrm{C}(17)-\mathrm{H}(17 \mathrm{C}) & 109.5 \\ \mathrm{H}(14 \mathrm{~B})-\mathrm{C}(14)-\mathrm{H}(14 \mathrm{C}) & 109.5 & \mathrm{H}(17 \mathrm{~A})-\mathrm{C}(17)-\mathrm{H}(17 \mathrm{C}) & 109.5 \\ \mathrm{C}(3)-\mathrm{C}(15)-\mathrm{H}(15 \mathrm{~A}) & 109.5 & \mathrm{H}(17 \mathrm{~B})-\mathrm{C}(17)-\mathrm{H}(17 \mathrm{C}) & 109.5 \\ \mathrm{C}(3)-\mathrm{C}(15)-\mathrm{H}(15 \mathrm{~B}) & 109.5 & \mathrm{C}(7)-\mathrm{O}(2)-\mathrm{C}(16) & 117.44(15) \\ \mathrm{H}(15 \mathrm{~A})-\mathrm{C}(15)-\mathrm{H}(15 \mathrm{~B}) & 109.5 & \mathrm{C}(9)-\mathrm{O}(3)-\mathrm{C}(17) & 116.36(15)\end{array}$

Table S6. Torsion angles $\left({ }^{\circ}\right.$ ) compound 4 (Symmetry transformations used to generate equivalent atoms).

$\begin{array}{lclc}C(12)-N(1)-C(1)-O(1) & -1.0(3) & C(4)-C(5)-C(6)-C(11) & 0.5(3) \\ C(4)-N(1)-C(1)-O(1) & 179.97(18) & C(4)-C(5)-C(6)-C(7) & -179.15(18) \\ C(12)-N(1)-C(1)-C(2) & 179.22(17) & C(11)-C(6)-C(7)-O(2) & -178.37(16) \\ C(4)-N(1)-C(1)-C(2) & 0.18(19) & C(5)-C(6)-C(7)-O(2) & 1.3(3) \\ O(1)-C(1)-C(2)-C(3) & -179.8(2) & C(11)-C(6)-C(7)-C(8) & 1.4(3) \\ N(1)-C(1)-C(2)-C(3) & 0.0(2) & C(5)-C(6)-C(7)-C(8) & -178.91(17) \\ O(1)-C(1)-C(2)-C(13) & -0.5(3) & O(2)-C(7)-C(8)-C(9) & -179.73(17) \\ N(1)-C(1)-C(2)-C(13) & 179.25(17) & C(6)-C(7)-C(8)-C(9) & 0.5(3) \\ C(1)-C(2)-C(3)-C(4) & -0.2(2) & C(7)-C(8)-C(9)-O(3) & 178.51(17) \\ C(13)-C(2)-C(3)-C(4) & -179.33(19) & C(7)-C(8)-C(9)-C(10) & -1.7(3) \\ C(1)-C(2)-C(3)-C(15) & 179.49(19) & O(3)-C(9)-C(10)-C(11) & -179.36(17) \\ C(13)-C(2)-C(3)-C(15) & 0.4(4) & C(8)-C(9)-C(10)-C(11) & 0.9(3) \\ C(12)-N(1)-C(4)-C(5) & -0.2(3) & C(7)-C(6)-C(11)-C(10) & -2.2(3) \\ C(1)-N(1)-C(4)-C(5) & 178.92(17) & C(5)-C(6)-C(11)-C(10) & 178.05(17) \\ C(12)-N(1)-C(4)-C(3) & -179.38(16) & C(7)-C(6)-C(11)-C(12) & 178.00(17) \\ C(1)-N(1)-C(4)-C(3) & -0.29(19) & C(5)-C(6)-C(11)-C(12) & -1.7(3) \\ C(2)-C(3)-C(4)-C(5) & -178.8(2) & C(9)-C(10)-C(11)-C(6) & 1.1(3) \\ C(15)-C(3)-C(4)-C(5) & 1.5(3) & C(9)-C(10)-C(11)-C(12) & -179.12(17) \\ C(2)-C(3)-C(4)-N(1) & 0.3(2) & C(4)-N(1)-C(12)-O(4) & 179.46(17) \\ C(15)-C(3)-C(4)-N(1) & -179.41(17) & C(1)-N(1)-C(12)-O(4) & 0.6(3) \\ N(1)-C(4)-C(5)-C(6) & 0.4(3) & C(4)-N(1)-C(12)-C(11) & -0.9(2) \\ C(3)-C(4)-C(5)-C(6) & 179.41(18) & C(1)-N(1)-C(12)-C(11) & -179.82(17)\end{array}$




$\begin{array}{lc}C(6)-C(11)-C(12)-O(4) & -178.56(19) \\ C(10)-C(11)-C(12)-O(4) & 1.7(3) \\ C(6)-C(11)-C(12)-N(1) & 1.8(2) \\ C(10)-C(11)-C(12)-N(1) & -177.94(16) \\ C(3)-C(2)-C(13)-C(14) & 89.5(3) \\ C(1)-C(2)-C(13)-C(14) & -89.6(3) \\ C(8)-C(7)-O(2)-C(16) & -3.0(3) \\ C(6)-C(7)-O(2)-C(16) & 176.79(17) \\ C(10)-C(9)-O(3)-C(17) & 10.4(3) \\ C(8)-C(9)-O(3)-C(17) & -169.79(17)\end{array}$


Table S7. Crystal data and structure refinement for 6. CCDC: 1421502

Identification code

Empirical formula

Formula weight

Temperature

Wavelength

Crystal system

Space group

Unit cell dimensions

Volume

Z

Density (calculated)

Absorption coefficient

$\mathrm{F}(000)$

Crystal size

Theta range for data collection

Index ranges

Reflections collected

Independent reflections

Completeness to theta $=25.242^{\circ}$

Absorption correction

Refinement method

Data / restraints / parameters

Goodness-of-fit on $\mathrm{F}^{2}$

Final R indices [I $>2 \operatorname{sigma}(\mathrm{I})]$

$\mathrm{R}$ indices (all data)

Extinction coefficient

Largest diff. peak and hole uwl010_0m

$\mathrm{C}_{14} \mathrm{H}_{14} \mathrm{~B} \mathrm{~N} \mathrm{O}_{2}$

239.07

100(2) K

$0.71073 \AA$

Triclinic

P -1

$\mathrm{a}=7.4434(5) \AA$

$\alpha=73.1530(10)^{\circ}$.

$\mathrm{b}=8.1430(5) \AA$

$\beta=83.8130(10)^{\circ}$.

$\mathrm{c}=10.3466(6) \AA$

592.88(6) $\AA^{3}$

2

$1.339 \mathrm{Mg} / \mathrm{m}^{3}$

$0.088 \mathrm{~mm}^{-1}$

252

$0.480 \times 0.082 \times 0.044 \mathrm{~mm}^{3}$

2.062 to $30.028^{\circ}$.

$-10<=\mathrm{h}<=10,-11<=\mathrm{k}<=11,-14<=\mathrm{l}<=14$

14651

$3468[\mathrm{R}(\mathrm{int})=0.0299]$

$100.0 \%$

SADABS

Full-matrix least-squares on $\mathrm{F}^{2}$

3468 / 0 / 163

1.046

$\mathrm{R} 1=0.0451, \mathrm{wR} 2=0.1327$

$\mathrm{R} 1=0.0605, \mathrm{wR} 2=0.1449$

$\mathrm{n} / \mathrm{a}$

0.508 and -0.253 e. $\AA^{-3}$ 
Table S8. Bond lengths $[\AA]$ and angles $\left[^{\circ}\right]$ for 6 (Symmetry transformations used to generate equivalent atoms).

\begin{tabular}{|c|c|c|c|}
\hline $\mathrm{O}(1)-\mathrm{B}(1)$ & $1.3568(14)$ & $\mathrm{C}(1)-\mathrm{C}(2)-\mathrm{C}(12)$ & $121.91(10)$ \\
\hline $\mathrm{O}(1)-\mathrm{H}(1 \mathrm{~A})$ & 0.8400 & $\mathrm{C}(2)-\mathrm{C}(3)-\mathrm{C}(4)$ & $108.78(10)$ \\
\hline $\mathrm{N}(1)-\mathrm{C}(1)$ & $1.4002(14)$ & $\mathrm{C}(2)-\mathrm{C}(3)-\mathrm{C}(14)$ & $128.76(11)$ \\
\hline $\mathrm{N}(1)-\mathrm{C}(4)$ & $1.4047(13)$ & $\mathrm{C}(4)-\mathrm{C}(3)-\mathrm{C}(14)$ & $122.44(10)$ \\
\hline $\mathrm{N}(1)-\mathrm{B}(1)$ & $1.4438(16)$ & $\mathrm{C}(5)-\mathrm{C}(4)-\mathrm{N}(1)$ & $121.74(10)$ \\
\hline $\mathrm{C}(1)-\mathrm{O}(2)$ & $1.2258(13)$ & $C(5)-C(4)-C(3)$ & $130.78(10)$ \\
\hline$C(1)-C(2)$ & $1.4802(16)$ & $\mathrm{N}(1)-\mathrm{C}(4)-\mathrm{C}(3)$ & $107.48(9)$ \\
\hline $\mathrm{B}(1)-\mathrm{C}(11)$ & $1.5499(18)$ & $C(4)-C(5)-C(6)$ & $121.67(10)$ \\
\hline $\mathrm{C}(2)-\mathrm{C}(3)$ & $1.3514(16)$ & $\mathrm{C}(4)-\mathrm{C}(5)-\mathrm{H}(5 \mathrm{~A})$ & 119.2 \\
\hline $\mathrm{C}(2)-\mathrm{C}(12)$ & $1.4937(16)$ & $\mathrm{C}(6)-\mathrm{C}(5)-\mathrm{H}(5 \mathrm{~A})$ & 119.2 \\
\hline$C(3)-C(4)$ & $1.4634(15)$ & $C(7)-C(6)-C(11)$ & $119.59(10)$ \\
\hline $\mathrm{C}(3)-\mathrm{C}(14)$ & $1.4952(15)$ & $C(7)-C(6)-C(5)$ & $120.89(10)$ \\
\hline$C(4)-C(5)$ & $1.3490(15)$ & $C(11)-C(6)-C(5)$ & $119.52(10)$ \\
\hline$C(5)-C(6)$ & $1.4566(15)$ & $\mathrm{C}(8)-\mathrm{C}(7)-\mathrm{C}(6)$ & $120.35(11)$ \\
\hline $\mathrm{C}(5)-\mathrm{H}(5 \mathrm{~A})$ & 0.9500 & $\mathrm{C}(8)-\mathrm{C}(7)-\mathrm{H}(7 \mathrm{~A})$ & 119.8 \\
\hline$C(6)-C(7)$ & $1.4049(16)$ & $\mathrm{C}(6)-\mathrm{C}(7)-\mathrm{H}(7 \mathrm{~A})$ & 119.8 \\
\hline$C(6)-C(11)$ & $1.4138(15)$ & $\mathrm{C}(7)-\mathrm{C}(8)-\mathrm{C}(9)$ & $120.28(11)$ \\
\hline $\mathrm{C}(7)-\mathrm{C}(8)$ & $1.3873(17)$ & $\mathrm{C}(7)-\mathrm{C}(8)-\mathrm{H}(8 \mathrm{~A})$ & 119.9 \\
\hline $\mathrm{C}(7)-\mathrm{H}(7 \mathrm{~A})$ & 0.9500 & $\mathrm{C}(9)-\mathrm{C}(8)-\mathrm{H}(8 \mathrm{~A})$ & 119.9 \\
\hline $\mathrm{C}(8)-\mathrm{C}(9)$ & $1.3939(18)$ & $\mathrm{C}(10)-\mathrm{C}(9)-\mathrm{C}(8)$ & $119.78(11)$ \\
\hline $\mathrm{C}(8)-\mathrm{H}(8 \mathrm{~A})$ & 0.9500 & $\mathrm{C}(10)-\mathrm{C}(9)-\mathrm{H}(9 \mathrm{~A})$ & 120.1 \\
\hline$C(9)-C(10)$ & $1.3838(18)$ & $\mathrm{C}(8)-\mathrm{C}(9)-\mathrm{H}(9 \mathrm{~A})$ & 120.1 \\
\hline $\mathrm{C}(9)-\mathrm{H}(9 \mathrm{~A})$ & 0.9500 & $\mathrm{C}(9)-\mathrm{C}(10)-\mathrm{C}(11)$ & $121.29(11)$ \\
\hline $\mathrm{C}(10)-\mathrm{C}(11)$ & $1.4024(16)$ & $\mathrm{C}(9)-\mathrm{C}(10)-\mathrm{H}(10 \mathrm{~A})$ & 119.4 \\
\hline $\mathrm{C}(10)-\mathrm{H}(10 \mathrm{~A})$ & 0.9500 & $\mathrm{C}(11)-\mathrm{C}(10)-\mathrm{H}(10 \mathrm{~A})$ & 119.4 \\
\hline$C(12)-C(13)$ & $1.5285(16)$ & $C(10)-C(11)-C(6)$ & $118.68(10)$ \\
\hline $\mathrm{C}(12)-\mathrm{H}(12 \mathrm{~A})$ & 0.9900 & $\mathrm{C}(10)-\mathrm{C}(11)-\mathrm{B}(1)$ & $121.90(10)$ \\
\hline $\mathrm{C}(12)-\mathrm{H}(12 \mathrm{~B})$ & 0.9900 & $\mathrm{C}(6)-\mathrm{C}(11)-\mathrm{B}(1)$ & $119.40(10)$ \\
\hline $\mathrm{C}(13)-\mathrm{H}(13 \mathrm{~A})$ & 0.9800 & $\mathrm{C}(2)-\mathrm{C}(12)-\mathrm{C}(13)$ & $113.07(10)$ \\
\hline $\mathrm{C}(13)-\mathrm{H}(13 \mathrm{~B})$ & 0.9800 & $\mathrm{C}(2)-\mathrm{C}(12)-\mathrm{H}(12 \mathrm{~A})$ & 109.0 \\
\hline $\mathrm{C}(13)-\mathrm{H}(13 \mathrm{C})$ & 0.9800 & $\mathrm{C}(13)-\mathrm{C}(12)-\mathrm{H}(12 \mathrm{~A})$ & 109.0 \\
\hline $\mathrm{C}(14)-\mathrm{H}(14 \mathrm{~A})$ & 0.9800 & $\mathrm{C}(2)-\mathrm{C}(12)-\mathrm{H}(12 \mathrm{~B})$ & 109.0 \\
\hline $\mathrm{C}(14)-\mathrm{H}(14 \mathrm{~B})$ & 0.9800 & $\mathrm{C}(13)-\mathrm{C}(12)-\mathrm{H}(12 \mathrm{~B})$ & 109.0 \\
\hline \multirow[t]{2}{*}{$\mathrm{C}(14)-\mathrm{H}(14 \mathrm{C})$} & 0.9800 & $\mathrm{H}(12 \mathrm{~A})-\mathrm{C}(12)-\mathrm{H}(12 \mathrm{~B})$ & 107.8 \\
\hline & & $\mathrm{C}(12)-\mathrm{C}(13)-\mathrm{H}(13 \mathrm{~A})$ & 109.5 \\
\hline $\mathrm{B}(1)-\mathrm{O}(1)-\mathrm{H}(1 \mathrm{~A})$ & 109.5 & $\mathrm{C}(12)-\mathrm{C}(13)-\mathrm{H}(13 \mathrm{~B})$ & 109.5 \\
\hline $\mathrm{C}(1)-\mathrm{N}(1)-\mathrm{C}(4)$ & $108.52(9)$ & $\mathrm{H}(13 \mathrm{~A})-\mathrm{C}(13)-\mathrm{H}(13 \mathrm{~B})$ & 109.5 \\
\hline $\mathrm{C}(1)-\mathrm{N}(1)-\mathrm{B}(1)$ & $128.83(9)$ & $\mathrm{C}(12)-\mathrm{C}(13)-\mathrm{H}(13 \mathrm{C})$ & 109.5 \\
\hline $\mathrm{C}(4)-\mathrm{N}(1)-\mathrm{B}(1)$ & $122.64(9)$ & $\mathrm{H}(13 \mathrm{~A})-\mathrm{C}(13)-\mathrm{H}(13 \mathrm{C})$ & 109.5 \\
\hline $\mathrm{O}(2)-\mathrm{C}(1)-\mathrm{N}(1)$ & $123.75(11)$ & $\mathrm{H}(13 \mathrm{~B})-\mathrm{C}(13)-\mathrm{H}(13 \mathrm{C})$ & 109.5 \\
\hline $\mathrm{O}(2)-\mathrm{C}(1)-\mathrm{C}(2)$ & $128.90(11)$ & $\mathrm{C}(3)-\mathrm{C}(14)-\mathrm{H}(14 \mathrm{~A})$ & 109.5 \\
\hline $\mathrm{N}(1)-\mathrm{C}(1)-\mathrm{C}(2)$ & $107.35(9)$ & $\mathrm{C}(3)-\mathrm{C}(14)-\mathrm{H}(14 \mathrm{~B})$ & 109.5 \\
\hline $\mathrm{O}(1)-\mathrm{B}(1)-\mathrm{N}(1)$ & $123.08(11)$ & $\mathrm{H}(14 \mathrm{~A})-\mathrm{C}(14)-\mathrm{H}(14 \mathrm{~B})$ & 109.5 \\
\hline $\mathrm{O}(1)-\mathrm{B}(1)-\mathrm{C}(11)$ & $122.01(10)$ & $\mathrm{C}(3)-\mathrm{C}(14)-\mathrm{H}(14 \mathrm{C})$ & 109.5 \\
\hline $\mathrm{N}(1)-\mathrm{B}(1)-\mathrm{C}(11)$ & $114.89(10)$ & $\mathrm{H}(14 \mathrm{~A})-\mathrm{C}(14)-\mathrm{H}(14 \mathrm{C})$ & 109.5 \\
\hline$C(3)-C(2)-C(1)$ & $107.86(10)$ & $\mathrm{H}(14 \mathrm{~B})-\mathrm{C}(14)-\mathrm{H}(14 \mathrm{C})$ & 109.5 \\
\hline $\mathrm{C}(3)-\mathrm{C}(2)-\mathrm{C}(12)$ & $130.22(11)$ & & \\
\hline
\end{tabular}


Table S9. Torsion angles $\left[{ }^{\circ}\right]$ for $\mathbf{6}$ (Symmetry transformations used to generate equivalent atoms).

\begin{tabular}{lc}
\hline $\mathrm{C}(4)-\mathrm{N}(1)-\mathrm{C}(1)-\mathrm{O}(2)$ & $-179.06(10)$ \\
$\mathrm{B}(1)-\mathrm{N}(1)-\mathrm{C}(1)-\mathrm{O}(2)$ & $1.84(18)$ \\
$\mathrm{C}(4)-\mathrm{N}(1)-\mathrm{C}(1)-\mathrm{C}(2)$ & $1.14(11)$ \\
$\mathrm{B}(1)-\mathrm{N}(1)-\mathrm{C}(1)-\mathrm{C}(2)$ & $-177.96(10)$ \\
$\mathrm{C}(1)-\mathrm{N}(1)-\mathrm{B}(1)-\mathrm{O}(1)$ & $-4.04(18)$ \\
$\mathrm{C}(4)-\mathrm{N}(1)-\mathrm{B}(1)-\mathrm{O}(1)$ & $176.98(10)$ \\
$\mathrm{C}(1)-\mathrm{N}(1)-\mathrm{B}(1)-\mathrm{C}(11)$ & $177.53(10)$ \\
$\mathrm{C}(4)-\mathrm{N}(1)-\mathrm{B}(1)-\mathrm{C}(11)$ & $-1.45(15)$ \\
$\mathrm{O}(2)-\mathrm{C}(1)-\mathrm{C}(2)-\mathrm{C}(3)$ & $179.20(11)$ \\
$\mathrm{N}(1)-\mathrm{C}(1)-\mathrm{C}(2)-\mathrm{C}(3)$ & $-1.02(12)$ \\
$\mathrm{O}(2)-\mathrm{C}(1)-\mathrm{C}(2)-\mathrm{C}(12)$ & $-1.58(18)$ \\
$\mathrm{N}(1)-\mathrm{C}(1)-\mathrm{C}(2)-\mathrm{C}(12)$ & $178.20(9)$ \\
$\mathrm{C}(1)-\mathrm{C}(2)-\mathrm{C}(3)-\mathrm{C}(4)$ & $0.49(12)$ \\
$\mathrm{C}(12)-\mathrm{C}(2)-\mathrm{C}(3)-\mathrm{C}(4)$ & $-178.64(11)$ \\
$\mathrm{C}(1)-\mathrm{C}(2)-\mathrm{C}(3)-\mathrm{C}(14)$ & $178.71(10)$ \\
$\mathrm{C}(12)-\mathrm{C}(2)-\mathrm{C}(3)-\mathrm{C}(14)$ & $-0.4(2)$ \\
$\mathrm{C}(1)-\mathrm{N}(1)-\mathrm{C}(4)-\mathrm{C}(5)$ & $178.99(10)$ \\
$\mathrm{B}(1)-\mathrm{N}(1)-\mathrm{C}(4)-\mathrm{C}(5)$ & $-1.84(16)$ \\
$\mathrm{C}(1)-\mathrm{N}(1)-\mathrm{C}(4)-\mathrm{C}(3)$ & $-0.85(11)$ \\
$\mathrm{B}(1)-\mathrm{N}(1)-\mathrm{C}(4)-\mathrm{C}(3)$ & $178.31(9)$ \\
$\mathrm{C}(2)-\mathrm{C}(3)-\mathrm{C}(4)-\mathrm{C}(5)$ & $-179.62(11)$ \\
$\mathrm{C}(14)-\mathrm{C}(3)-\mathrm{C}(4)-\mathrm{C}(5)$ & $2.02(18)$ \\
$\mathrm{C}(2)-\mathrm{C}(3)-\mathrm{C}(4)-\mathrm{N}(1)$ & $0.20(12)$ \\
$\mathrm{C}(14)-\mathrm{C}(3)-\mathrm{C}(4)-\mathrm{N}(1)$ & $-178.15(9)$ \\
$\mathrm{N}(1)-\mathrm{C}(4)-\mathrm{C}(5)-\mathrm{C}(6)$ & $2.95(17)$ \\
$\mathrm{C}(3)-\mathrm{C}(4)-\mathrm{C}(5)-\mathrm{C}(6)$ & $-177.25(10)$ \\
$\mathrm{C}(4)-\mathrm{C}(5)-\mathrm{C}(6)-\mathrm{C}(7)$ & $179.34(10)$ \\
$\mathrm{C}(4)-\mathrm{C}(5)-\mathrm{C}(6)-\mathrm{C}(11)$ & $-0.49(16)$ \\
$\mathrm{C}(11)-\mathrm{C}(6)-\mathrm{C}(7)-\mathrm{C}(8)$ & $1.59(17)$ \\
$\mathrm{C}(5)-\mathrm{C}(6)-\mathrm{C}(7)-\mathrm{C}(8)$ & $-178.25(10)$ \\
$\mathrm{C}(6)-\mathrm{C}(7)-\mathrm{C}(8)-\mathrm{C}(9)$ & $-0.66(18)$ \\
$\mathrm{C}(7)-\mathrm{C}(8)-\mathrm{C}(9)-\mathrm{C}(10)$ & $-0.67(18)$ \\
$\mathrm{C}(8)-\mathrm{C}(9)-\mathrm{C}(10)-\mathrm{C}(11)$ & $1.08(18)$ \\
$\mathrm{C}(9)-\mathrm{C}(10)-\mathrm{C}(11)-\mathrm{C}(6)$ & $-0.15(17)$ \\
$\mathrm{C}(9)-\mathrm{C}(10)-\mathrm{C}(11)-\mathrm{B}(1)$ & $-178.59(10)$ \\
$\mathrm{C}(7)-\mathrm{C}(6)-\mathrm{C}(11)-\mathrm{C}(10)$ & $-1.18(16)$ \\
$\mathrm{C}(5)-\mathrm{C}(6)-\mathrm{C}(11)-\mathrm{C}(10)$ & $178.66(9)$ \\
$\mathrm{C}(7)-\mathrm{C}(6)-\mathrm{C}(11)-\mathrm{B}(1)$ & $177.31(10)$ \\
$\mathrm{C}(5)-\mathrm{C}(6)-\mathrm{C}(11)-\mathrm{B}(1)$ & $-2.85(16)$ \\
$\mathrm{O}(1)-\mathrm{B}(1)-\mathrm{C}(11)-\mathrm{C}(10)$ & $3.71(17)$ \\
$\mathrm{N}(1)-\mathrm{B}(1)-\mathrm{C}(11)-\mathrm{C}(10)$ & $-177.85(10)$ \\
$\mathrm{O}(1)-\mathrm{B}(1)-\mathrm{C}(11)-\mathrm{C}(6)$ & $-174.73(10)$ \\
$\mathrm{N}(1)-\mathrm{B}(1)-\mathrm{C}(11)-\mathrm{C}(6)$ & \\
& \\
& \\
&
\end{tabular}


Table S9. Hydrogen bonds for $6\left[\AA\right.$ and $\left.^{\circ}\right]$.

\begin{tabular}{lcccc}
\hline D-H...A & $\mathrm{d}(\mathrm{D}-\mathrm{H})$ & $\mathrm{d}(\mathrm{H} \ldots \mathrm{A})$ & $\mathrm{d}(\mathrm{D} \ldots \mathrm{A})$ & $<(\mathrm{DHA})$ \\
\hline $\mathrm{O}(1)-\mathrm{H}(1 \mathrm{~A}) \ldots \mathrm{O}(2)$ & 0.84 & 2.23 & $2.9218(13)$ & 139.2 \\
$\mathrm{O}(1)-\mathrm{H}(1 \mathrm{~A}) \ldots \mathrm{O}(2) \# 1$ & 0.84 & 2.38 & $2.9550(12)$ & 125.7
\end{tabular}

Symmetry transformations used to generate equivalent atoms:

\#1 -x+2,-y+1,-z+2 